University of Tennessee Health Science Center

UTHSC Digital Commons

\title{
Factors Associated with African American Women's Sexual Health and Risk Behavior: A Mixed Methods Study
}

\author{
Melody N. Waller \\ University of Tennessee Health Science Center
}

Follow this and additional works at: https://dc.uthsc.edu/dissertations

Part of the Behavior and Behavior Mechanisms Commons, Gender and Sexuality Commons, Medicine and Health Commons, Race and Ethnicity Commons, and the Women's Health Commons

\section{Recommended Citation}

Waller, Melody N. (http://orcid.org/0000-0002-3626-8426), "Factors Associated with African American Women's Sexual Health and Risk Behavior: A Mixed Methods Study" (2016). Theses and Dissertations (ETD). Paper 394. http://dx.doi.org/10.21007/etd.cghs.2016.0399.

This Dissertation is brought to you for free and open access by the College of Graduate Health Sciences at UTHSC Digital Commons. It has been accepted for inclusion in Theses and Dissertations (ETD) by an authorized administrator of UTHSC Digital Commons. For more information, please contact jwelch30@uthsc.edu. 


\title{
Factors Associated with African American Women's Sexual Health and Risk Behavior: A Mixed Methods Study
}

\begin{abstract}
Introduction: When looking at the health status of our nation, an examination of sexual and reproductive health reveals that there are multiple conditions related to sexual behavior posing a burden on the health of African American women (AAW). Disproportionate rates of sexually transmitted infections, unintended/ teen pregnancy, and sexual violence in this group reveal the need to integrate a broader construct to address the multiple factors known to contribute to disparities in sexual and reproductive health affecting AAW. Current health promotion efforts including the encouragement of abstinence and condom use fail to address the multiple components involved in sexual health and/or risk behavior and have been unsuccessful in curtailing disparities in this population. Recently, the concept of sexual health has increasingly been recognized for its potential to improve population health for various groups by addressing pertinent emotional, relational, and sexual aspects involved in individuals' sexual behavior, decision making, and negotiation. The Sexual Health Model is used in this study, to identify multiple dimensions involved in AAW's sexual health and participation in behaviors known to increase their sexual risk.

Methods: A convergent parallel mixed methods design was used in this study to better understand AAW's sexual health and risk behavior. Quantitative (QUAN) methods using questionnaires were carried out on the full study sample $(\mathrm{N}=50)$ to describe associations between selected emotional and relational factors (spirituality/religiosity, depressive symptomology, and gender inequality) and AAWs sexual health and risk behavior. In addition, qualitative (QUAL) methods, which included the completion of focus group interviews, were conducted for a subsample $(n=19)$ of study participants to examine AAWs perceptions of sexual health. Respondent validation procedures were also carried out on a subset of the QUAL study sample to ensure the accuracy of thematic content analysis. Analysis for QUAN data was conducted using SAS 9.4 and for QUAL data using NVivo 10. Individual analyses were integrated to corroborate findings for a mixed methods interpretation.
\end{abstract}

Results: Study participants (mean age $=38.5$ ) reported having good overall sexual health and low sexual risk. Significant associations were identified among specified emotional and relational factors and both outcome variables. Sexual health was negatively correlated with depressive symptomology $(r=-.19, p<$ $.20)$ and gender inequality $(r=-.25, p<.10)$. Additionally, lower depressive symptomology and gender inequality demonstrate predictive properties for AAWs sexual health $\left(R^{2}=.12, p<0.05\right)$. Examination of sexual risk behavior reveal a positive association with gender inequality $(r=.19, p<.20)$. Spirituality/ religiosity were not correlated with either outcome variable. QUAL study results reveal five organizing themes that emerged from focus group data, including: 1) "The new normal"; 2) having to compromise; 3) taking responsibility; 4) securing an attachment; and 5) resolving ambiguity.

Discussion/conclusion: By taking a holistic approach to better understand AAW's sexual behavior, this study provided important insights into the multiple factors involved in achieving an optimal level of sexual health for these women. In this study, women's personal accounts acknowledged participation in risky sexual behavior that was motivated by their intuitive feelings or the existing dynamic within their intimate relationships. Additionally, for women having the ability to make decisions within relationships, did not consistently translate to making sexually healthy decisions. Taking a broader approach toward sexual health may significantly contribute to the improvement of disparate health outcomes among AAW. Pertinent emotional, relational, and sexual factors involved in women's sexual health and behavior must be addressed in strategies to reduce AAWs sexual risk and yield sustainable behavior change. 


\section{Document Type}

Dissertation

\section{Degree Name}

Doctor of Philosophy (PhD)

\section{Program}

Nursing Science

\section{Research Advisor}

Mona N. Wicks, Ph.D.

\section{Keywords}

African American women, depressive symptomology, gender inequality, mixed methodology, religiosity/ spirituality, sexual health

\section{Subject Categories}

Behavior and Behavior Mechanisms | Gender and Sexuality | Medicine and Health | Medicine and Health Sciences | Psychiatry and Psychology | Public Health | Race and Ethnicity | Social and Behavioral Sciences | Sociology | Women's Health 
Factors Associated with African American Women's Sexual Health and Risk Behavior: A Mixed Methods Study

\author{
A Dissertation \\ Presented for \\ The Graduate Studies Council \\ The University of Tennessee \\ Health Science Center \\ In Partial Fulfillment \\ Of the Requirements for the Degree \\ Doctor of Philosophy \\ From The University of Tennessee
}

By

Melody N. Waller

May 2016 
Copyright (C) 2016 by Melody N. Waller. All rights reserved. 


\section{DEDICATION}

I dedicate this work to my husband, Quinton for helping me to maintain this balancing act, pushing me when I need a gentle nudge, and always being there to support. I am grateful to my children, Brandyn and Bryce for expecting me to be Momma first and everything else second; I love you both! And I am grateful to my parents, Louise, Ann, and Leroy, for doing everything in your power to help me be successful—and, to my brother, Mike for your listening ear and constant encouragement. 


\section{ACKNOWLEDGEMENTS}

I would like to thank the 50 African American women who gladly and sincerely shared their intimate life experiences with me. I also would like to thank Dr. Latrice Pichon for providing me a different perspective and being both an inspiration and motivation to contribute to the health of African American women.

I would also like to thank Dr. Patricia Cowan and Dr. Betsy Tolley for equipping me with a solid foundation to make sound, rigorous, and considerate contributions to the science of nursing. I would also like to thank Dr. Wendy Likes for my introduction to the science of women's health, your personal and professional support, and contribution of time. I would also like to thank Dr. Carolyn Graff for imparting your knowledge of qualitative research, showing me how to "stretch" my thinking, and accepting the distinction of my thoughts.

I would also like to thank Dr. Mona Wicks, my dissertation committee chairperson, for your unwavering support, your depth of knowledge, and extreme compassion. You have been the ultimate example of both a nurse leader and researcher and my greatest advocate. Your kindness, empathy, and contribution of endless hours and energy on my behalf are greatly appreciated.

Lastly, I would like to acknowledge the Johnson \& Johnson/American Association of Colleges of Nursing Minority Nurse Faculty Scholars Program and the Beta Theta Chapter of Sigma Theta Tau International Honor Society of Nursing for awarding me with scholarships to support my dissertation research. 


\begin{abstract}
Introduction: When looking at the health status of our nation, an examination of sexual and reproductive health reveals that there are multiple conditions related to sexual behavior posing a burden on the health of African American women (AAW).

Disproportionate rates of sexually transmitted infections, unintended/teen pregnancy, and sexual violence in this group reveal the need to integrate a broader construct to address the multiple factors known to contribute to disparities in sexual and reproductive health affecting AAW. Current health promotion efforts including the encouragement of abstinence and condom use fail to address the multiple components involved in sexual health and/or risk behavior and have been unsuccessful in curtailing disparities in this population. Recently, the concept of sexual health has increasingly been recognized for its potential to improve population health for various groups by addressing pertinent emotional, relational, and sexual aspects involved in individuals' sexual behavior, decision making, and negotiation. The Sexual Health Model is used in this study, to identify multiple dimensions involved in AAW's sexual health and participation in behaviors known to increase their sexual risk.
\end{abstract}

Methods: A convergent parallel mixed methods design was used in this study to better understand AAW's sexual health and risk behavior. Quantitative (QUAN) methods using questionnaires were carried out on the full study sample $(\mathrm{N}=50)$ to describe associations between selected emotional and relational factors (spirituality/religiosity, depressive symptomology, and gender inequality) and AAWs sexual health and risk behavior. In addition, qualitative (QUAL) methods, which included the completion of focus group interviews, were conducted for a subsample $(n=19)$ of study participants to examine AAWs perceptions of sexual health. Respondent validation procedures were also carried out on a subset of the QUAL study sample to ensure the accuracy of thematic content analysis. Analysis for QUAN data was conducted using SAS 9.4 and for QUAL data using NVivo 10. Individual analyses were integrated to corroborate findings for a mixed methods interpretation.

Results: Study participants (mean age $=38.5$ ) reported having good overall sexual health and low sexual risk. Significant associations were identified among specified emotional and relational factors and both outcome variables. Sexual health was negatively correlated with depressive symptomology $(r=-.19, \mathrm{p}<.20)$ and gender inequality $(\mathrm{r}=-.25, \mathrm{p}<.10)$. Additionally, lower depressive symptomology and gender inequality demonstrate predictive properties for AAWs sexual health $\left(\mathrm{R}^{2}=.12, \mathrm{p}<0.05\right)$. Examination of sexual risk behavior reveal a positive association with gender inequality $(\mathrm{r}=.19, \mathrm{p}<.20)$. Spirituality/religiosity were not correlated with either outcome variable. QUAL study results reveal five organizing themes that emerged from focus group data, including: 1) "The new normal"; 2) having to compromise; 3) taking responsibility; 4) securing an attachment; and 5) resolving ambiguity.

Discussion/conclusion: By taking a holistic approach to better understand AAW's sexual behavior, this study provided important insights into the multiple factors 
involved in achieving an optimal level of sexual health for these women. In this study, women's personal accounts acknowledged participation in risky sexual behavior that was motivated by their intuitive feelings or the existing dynamic within their intimate relationships. Additionally, for women having the ability to make decisions within relationships, did not consistently translate to making sexually healthy decisions. Taking a broader approach toward sexual health may significantly contribute to the improvement of disparate health outcomes among AAW. Pertinent emotional, relational, and sexual factors involved in women's sexual health and behavior must be addressed in strategies to reduce AAWs sexual risk and yield sustainable behavior change. 


\section{TABLE OF CONTENTS}

CHAPTER 1. INTRODUCTION ..................................................................................

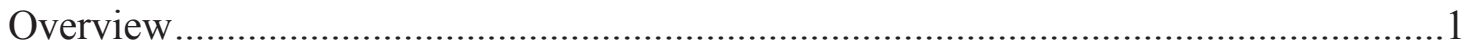

Purpose of the Study ........................................................................................ 4

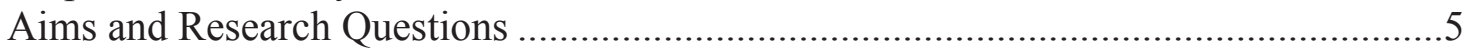

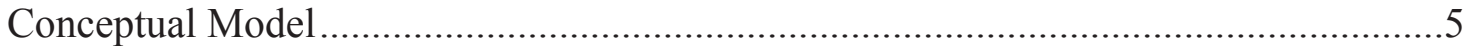

Definitions of Major Concepts and General Definitions .......................................10

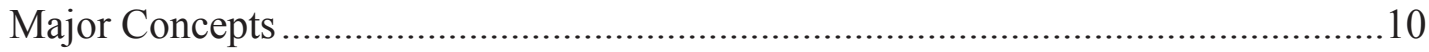

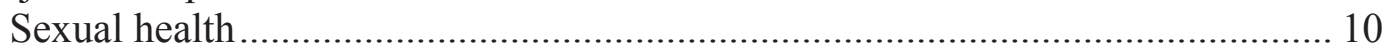

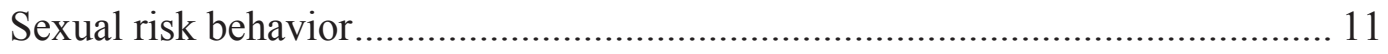

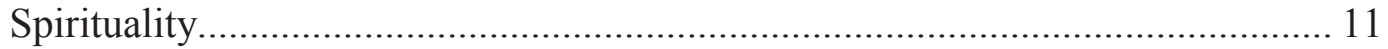

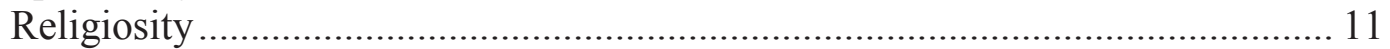

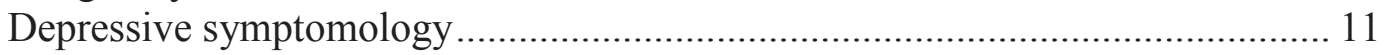

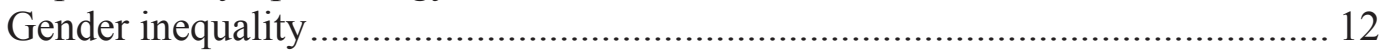

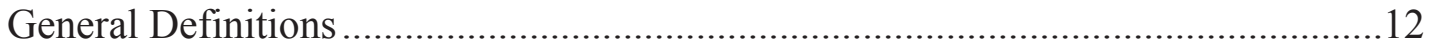

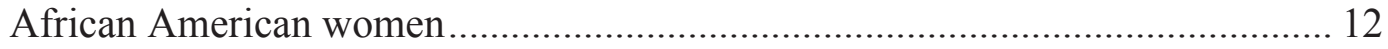

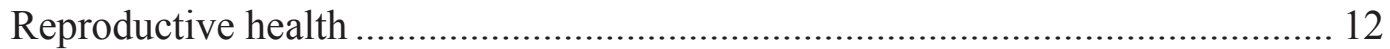

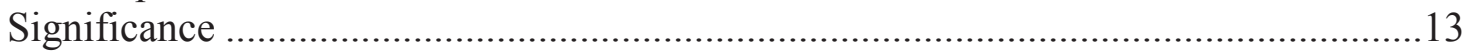

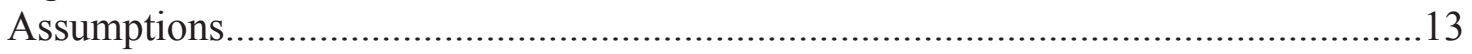

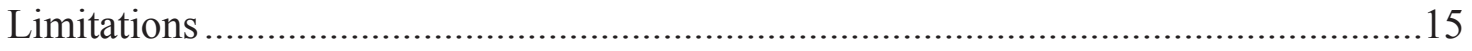

CHAPTER 2. REVIEW OF LITERATURE ...............................................................16

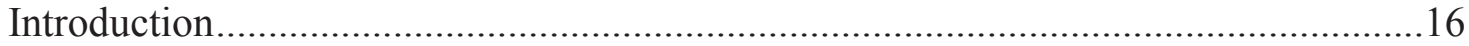

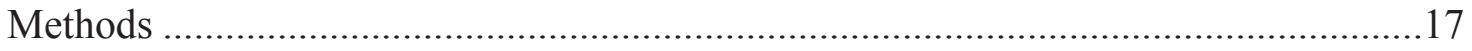

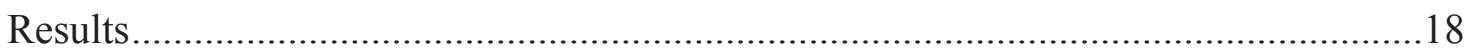

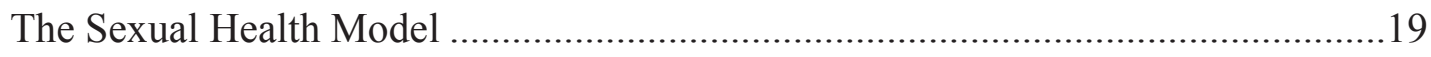

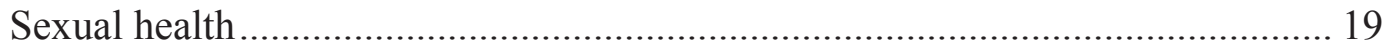

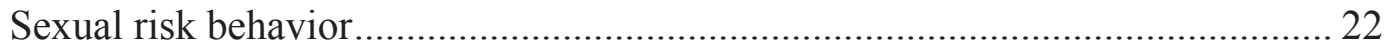

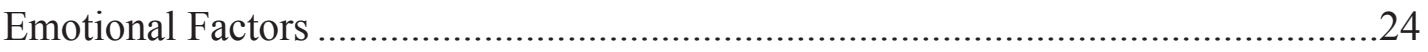

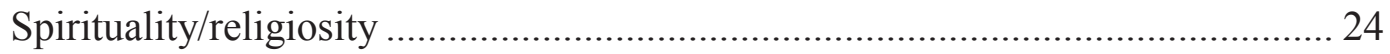

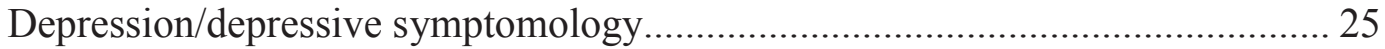

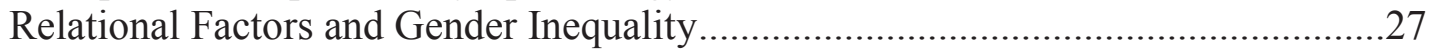

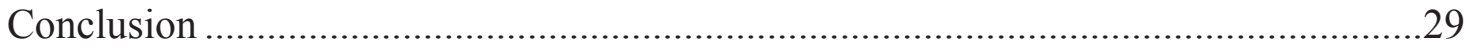

CHAPTER 3. METHODOLOGY .................................................................................30

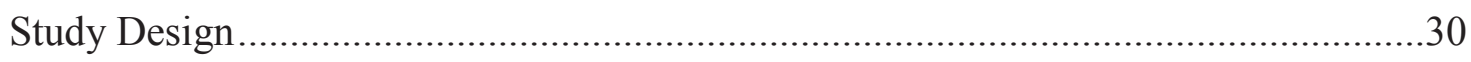

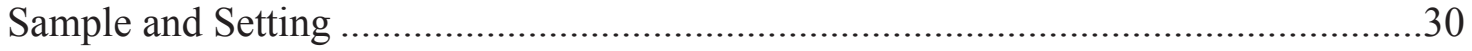

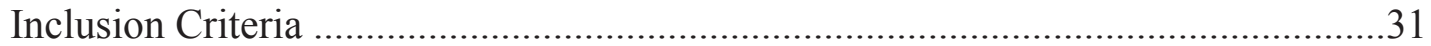

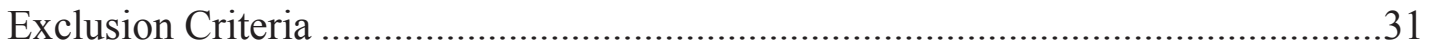

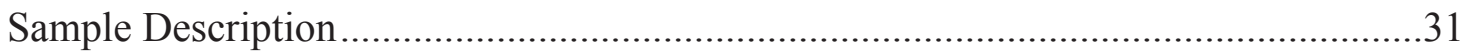

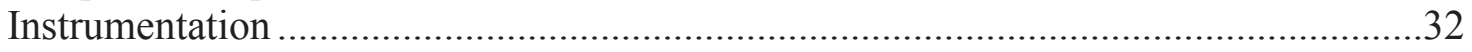

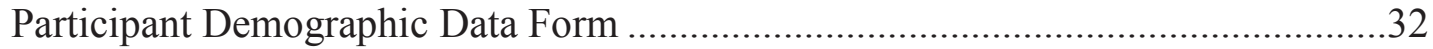

The Sexual Quality of Life-Female (SQOL-F) Questionnaire.................................33

The Sexual Activities and Attitudes Questionnaire (SAAQ) ....................................34 
The Brief Multidimensional Measure of Religiousness/Spirituality (BMMRS) .......36

The Center for Epidemiologic Studies Depression Scale Revised ...........................37

The Sexual Relationship Power Scale ....................................................................40

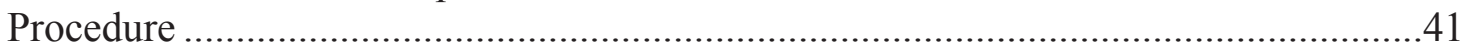

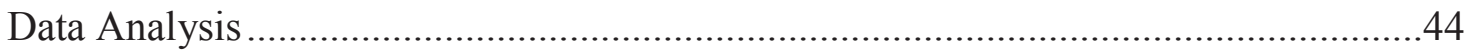

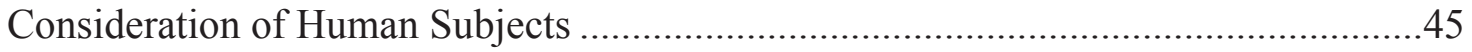

CHAPTER 4. RESULTS ................................................................................47

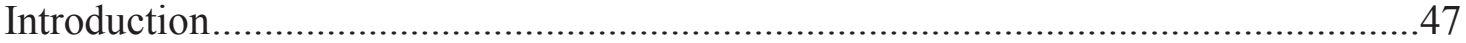

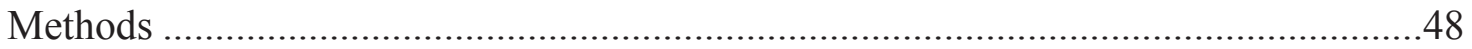

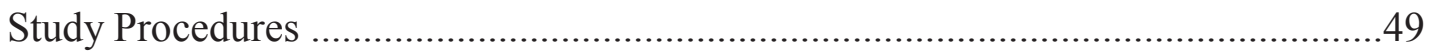

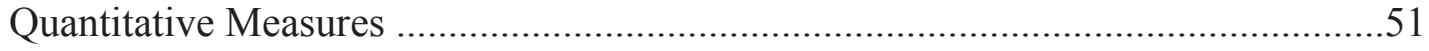

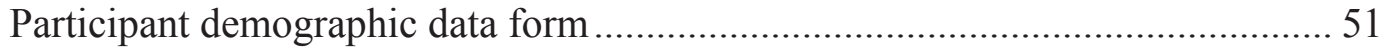

The sexual quality of life -female (SQOL-F) questionnaire .............................. 51

The sexual activities and attitudes questionnaire (SAAQ) ............................... 52

The brief multidimensional measure of religiousness / spirituality (BMMRS) ... 52

The Center for Epidemiologic Studies depression scale revised (CESD-R) ........ 53

The sexual relationship power scale (SRPS) ….............................................. 53

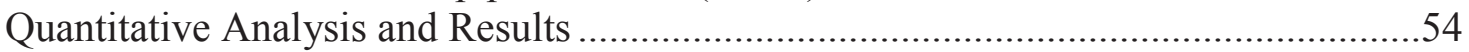

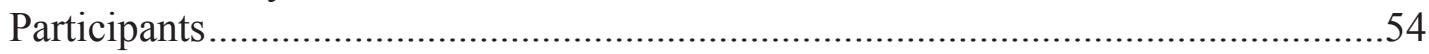

Sexual Health, Sexual Risk Behavior, Depressive Symptomology, Spiritualty /

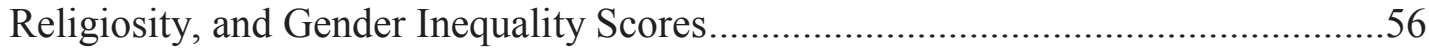

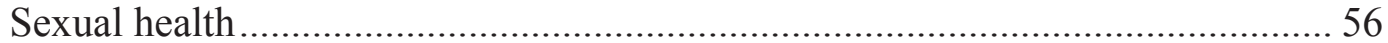

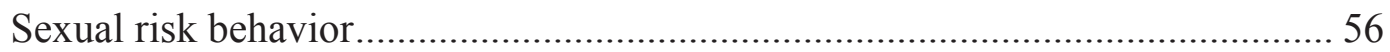

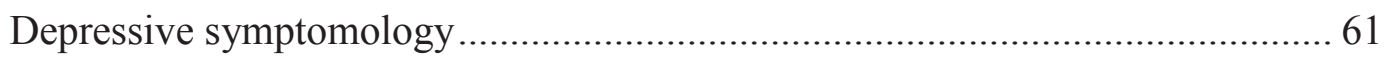

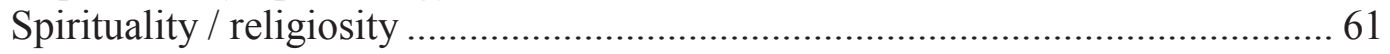

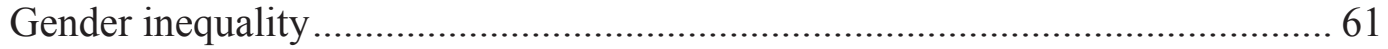

Relationships among Sexual Health, Sexual Risk Behavior, Depressive

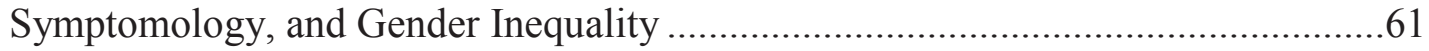

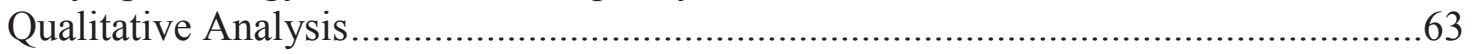

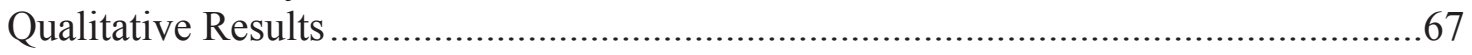

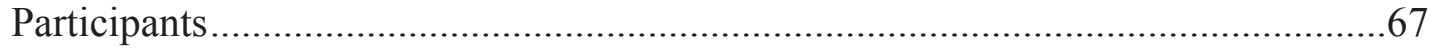

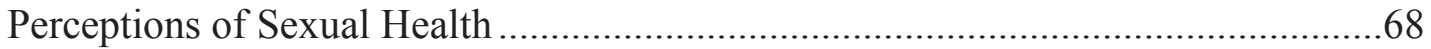

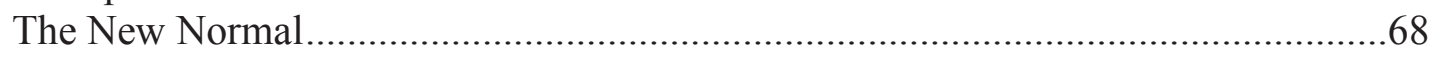

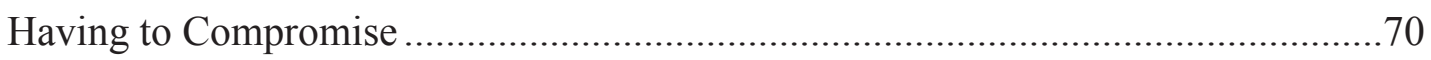

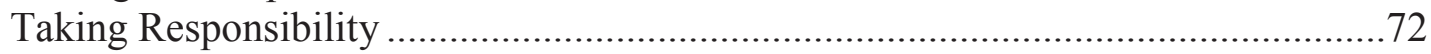

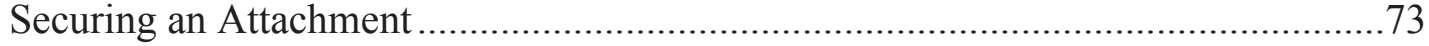

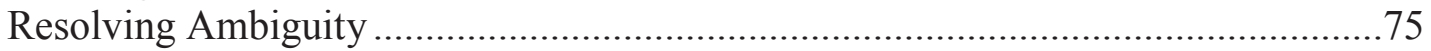

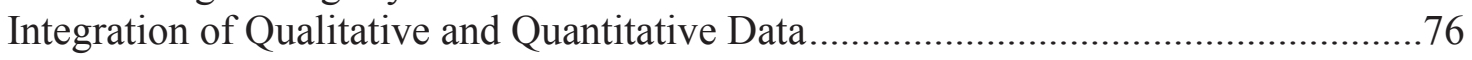

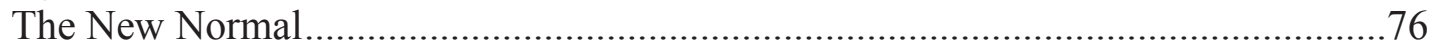

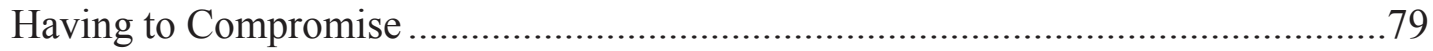

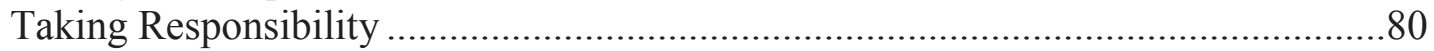

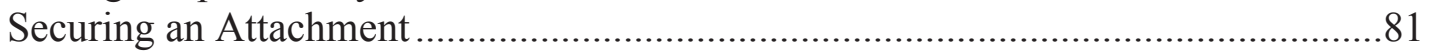

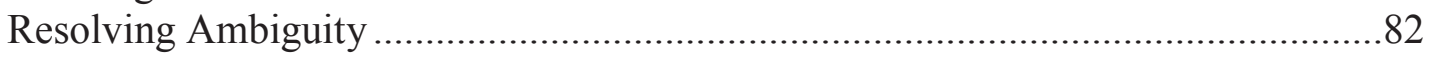

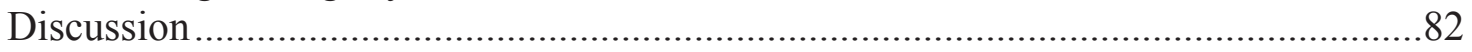

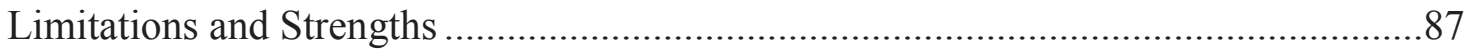




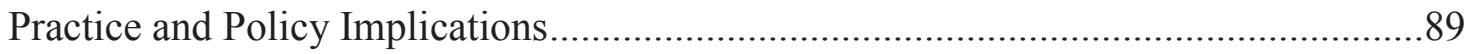

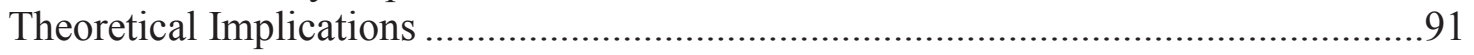

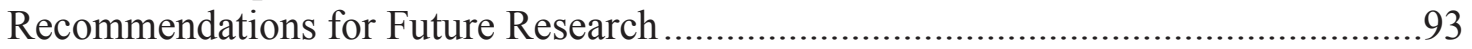

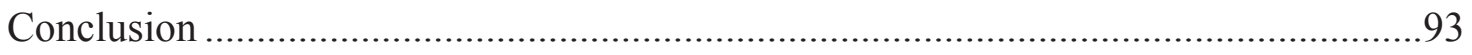

LIST OF REFERENCES ..................................................................................95

APPENDIX A. INSTITUTIONAL REVIEW BOARD LETTER OF

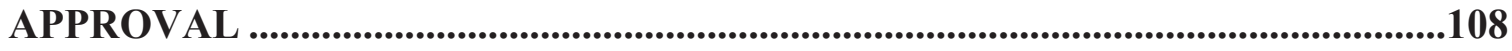

APPENDIX B. INSTITUTIONAL REVIEW BOARD APPROVED

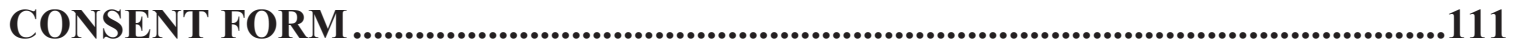

APPENDIX C. PARTICIPANT DEMOGRAPHIC FORM...................................119

APPENDIX D. DEFINITIONS OF QUALITATIVE CODES AND

CATEGORIES ....................................................................................................................................123

VITA 


\section{LIST OF TABLES}

Table 3-1. Description of Brief Multidimensional Measure of Religiousness /

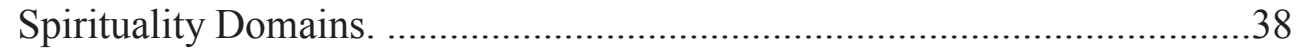

Table 3-2. Instrument Reliability and Completion Times.........................................42

Table 4-1. Demographic Characteristics as Reported by a Sample of African American Women.

Table 4-2. Sexual Health Characteristics as Reported by a Sample of African American Women.

Table 4-3. Substance Use as Reported by a Sample of African American Women. .....58

Table 4-4. General and Mental Health Characteristics as Reported by a Sample of African American Women.

Table 4-5. Self-Reported Sexual Health, Risk Behavior, Emotional and Relational Factors as Reported by a Sample of 50 African American Women. .60

Table 4-6. Relationships among Sexual Health, Sexual Risk Behavior, Depressive Symptomology, and Gender Inequality as Reported by a Sample of 50 African American Women.

Table 4-7. Multiple Regression Model Predicting Sexual Health for a Sample of 50 African American Women.

Table 4-8. $\quad$ Self-Reported Sexual Health, Risk Behavior, Emotional and Relational Factors as Reported by a Sample of 47 African American Women.

Table 4-9. Relationships among Sexual Health, Sexual Risk Behavior, Depressive Symptomology, and Gender Inequality as Reported by a Sample of 47 African American Women.

Table 4-10. Thematic Structure of Perceptions of Sexual Health for a Sample of African American Women $(\mathrm{n}=19)$.

Table 4-11. Integration of Quantitative Variables with Qualitative Themes. .77

Table 4-12. Integration of Quantitative Inferences with Qualitative Themes. .78 


\section{LIST OF FIGURES}

Figure 1-1. The Sexual Health Model. ....................................................................

Figure 1-2. Middle Range Theory Derived from the Sexual Health Model....................14

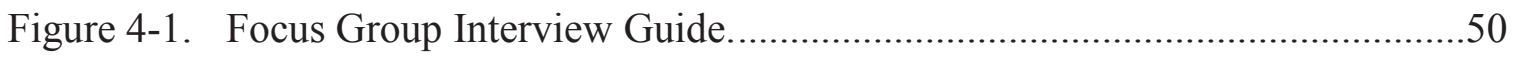




\section{CHAPTER 1. INTRODUCTION}

\section{Overview}

In recent years the concept of sexual health has increasingly been recognized for its potential to improve population health and has become an essential component of health promotion. With the burden of conditions such as sexually transmitted infections (STIs), human immunodeficiency virus (HIV)/acquired immunodeficiency syndrome (AIDS), and unintended pregnancies continuing to threaten the health of young Americans, the need to integrate a broader construct beyond reproductive health is required to encompass the wide range of factors contributing to the prevalence of these public health issues (Ford, Barnes, Rompalo, \& Hook III, 2013). Estimates from the Centers for Disease Control and Prevention (2014f) reveal that STIs occur at almost 20 million cases annually, with young people between the ages of $15-24$ representing $50 \%$ of new cases. Nearly 50,000 people within the US are infected with HIV each year, adding to the over one million Americans currently living with the virus. Addressing the broad construct of sexual health, rather than reproductive health, may be the key to addressing HIV infection rates, STIs in general, and unintended pregnancies. The failure to address sexual health may negatively influence health outcomes such as the progression of HIV to AIDS, which caused 15,500 deaths in 2010 (Centers for Disease Control and Prevention, 2014c). Like trends describing the incidence of STIs, unintended pregnancy rates and teen birth rates reveal the potential impact that improving sexual health could have on health promotion. With over half of pregnancies in the US being unintended, reducing the rate at which these occur is a national public health goal (Guttmacher Institute, 2013).

The definition of sexual health has been refined based upon its influence on the health status of Americans and identified public health priorities. Most recently, the Centers for Disease Control and Prevention and Health Resources and Services Administration (HRSA) Advisory Committee on HIV, Viral Hepatitis, and STD Prevention and Treatment defined sexual health as "... a state of well-being in relation to sexuality across the life span that involves physical, emotional, mental, social, and spiritual dimensions. Sexual health is an intrinsic element of human health and is based on a positive, equitable, and respectful approach to sexuality, relationships, and reproduction, that is free of coercion, fear, discrimination, stigma, shame, and violence (2012, p. 14).”

Sexual health, as defined by these and other public health organizations, emphasizes an individual's sexual well-being, instead of disease prevention. Achieving this state of health is likely influenced by a myriad of factors that include biological risk, genetic predisposition, mental health, violence, and sexual attitudes or behavior (World Health Organization, 2002). Addressing sexual health requires a holistic health-focused approach with interventions that integrate sexuality and that are carried out at the individual, community, and healthcare system levels (Douglas \& Fenton, 2013; World Health Organization, 2002). 
While the theoretical definition of sexual health is evolving, a similar evolution is taking place in the research, surveillance, and practical application of interventions to address this important health issue. Traditionally, sexual health has been measured using participation in risky sexual behaviors and incidence of health problems related to these behaviors such as STI and HIV/AIDS infection, teen and unintended pregnancy, or sexual violence (Douglas \& Fenton, 2013; Robinson, Bockting, Rosser, Miner, \& Coleman, 2002). Sexual behaviors known to increase the risk of STI, HIV/AIDS infection, and similar health problems include the following: having unprotected vaginal or anal sex, participating in oral sex, using illegal substances, and sharing drug use equipment (Centers for Disease Control and Prevention, 2014a). Trading sexual favors for money or other means of support, concurrent sexual partnerships, and being involved in actual or potential partner violence are additional behaviors that have been cited as increasing risk (Whyte, 2005). Measuring sexual health using indicators that are conceptually congruent with its definition is needed to adequately characterize the many issues that may influence sexuality and sexual behavior (Ivankovich, Leichliter, \& Douglas Jr., 2013).

Sexual health promotion has been largely focused on STI, HIV, and pregnancy prevention and concentrated heavily on behavioral and social interventions that are directed toward US populations deemed most vulnerable (Centers for Disease Control and Prevention, 2014e). Despite these efforts developed to address disparities in sexual and reproductive health, HIV and other STIs continue to be significant national health problems, particularly among specific subpopulations or social groups (Reid, Dovidio, Ballester, \& Johnson, 2014). Although African American women (AAW) have experienced a $21 \%$ decline in HIV infection rates since 2008, this group continues to represent the highest proportion of infected women with rates 20 times higher than that of white women and nearly 5 times that of Latina women (Carter et al., 2013; Centers for Disease Control and Prevention, 2014c). Similar disparities can be seen in recent teen birth rates, as well as, gonorrhea and chlamydia infection rates among AAW (Centers for Disease Control and Prevention, 2014f; Martin, Hamilton, Ventura, Osterman, \& Mathews, 2013). Teen birth rates for African Americans in 2011 were twice the rate of non-Hispanic white teens (Ventura, Hamilton, \& Matthews, 2014; Ventura et al., 2011). Additionally, 2011 STI surveillance data reveal gonorrhea infection rates among AAW were over 15 times that of white women and chlamydia rates were over six times higher than the rate among white women (Centers for Disease Control and Prevention, 2014a).

Existing STI and HIV prevention strategies such as basic sex education and condom use have proven unsuccessful in ameliorating the disproportionate burden of disease and negative sexual health outcomes experienced among AAW (J. Williams, Wyatt, \& Wingood, 2010). Consequently, trends reveal an identifiable gap between existing reproductive health strategies and the potential of integrating a broader perspective of sexual health. By including sex education with strategies focused on addressing relational factors, communication, and access to reproductive and sexual health services, a sexual health perspective could complement existing preventive strategies (Ford et al., 2013). 
Investigators who developed current prevention strategies have acknowledged the need to address emotional and socio-cultural factors that promote participation in sexual risk behaviors within this population, particularly engaging in unprotected sex (Paxton, Williams, Bolden, Guzman, \& Harawa, 2013). Current strategies targeting condom use and similar preventative approaches that focus on decision making fail to distinguish relational and sexual factors that may influence participation in 'risky' behavior. Gender inequality, a concept that moderates difficulty asserting or negotiating condom use and/or preferences not to use condoms in monogamous relationships, may in part explain some AAW's ongoing participation in unprotected sexual activity (Paxton et al., 2013). Acknowledging the impact that power and gender issues may have on sexual risk and sexual health within this population is essential to developing successful prevention strategies and interventions. For this reason, using theories or conceptual frameworks that include emotional, relational (e.g., gender and power), and sexual factors that influence both sexual risk behavior and sexual health are necessary to fully address the range of factors underlying the existing disparity in STI, HIV infection, and teen birth rates affecting AAW.

In addition to underlying factors that are largely relational in nature, behavior patterns that involve engaging in unprotected sex may be emotional and suggest the presence of conditions that are psychological or psychosocial (Robinson et al., 2002). In a meta-analysis, Lennon, Huedo-Medina, Gerwien, and Johnson (2012) found significant changes in sexual risk behavior in samples largely composed of young adult AAW experiencing depressive symptoms who participated in HIV prevention interventions. Within studies included in this meta-analysis, AAW who had higher depression levels at baseline demonstrated greater sexual risk behavior change at study completion, suggesting that a reduction in depression levels may decrease participation in behaviors that increase sexual risk (Lennon et al., 2012). Similarly, another study of African American adolescent females suggested a predictive relationship between higher levels of depressive symptoms and recent unprotected sexual activity (Seth et al., 2011). The results of the two aforementioned studies support the need to examine depression symptoms as an emotional factor significantly influencing sexual health and participation in sexual risk behaviors.

Addressing significant aspects of sexual health must also consider exposure to cultural traditions that shape behavior from an ethical or moral perspective. Spirituality and religiosity have both been identified as emotional factors that influence health outcomes; both concepts are based primarily upon personal beliefs and moral values (Robinson et al., 2002). Religion has been cited as having an important influence on the lives of many African Americans and has been identified as a protective factor, often preventing young AAW's participation in risky sexual behaviors (Montgomery et al., 2014; Thomas \& Freeman, 2011). Spirituality, which may or may not include formal religious typologies, has been recognized as having a similar impact on health behaviors within this population (Thomas \& Freeman, 2011). For these reasons, many prevention efforts designed to address the disparity in HIV infection among AAW include faithbased components (Montgomery et al., 2014). However, much of the existing work in 
this area has focused on adolescents, thus exposing the need to explore associations between spirituality and/or religiosity and sexual risk behavior in adult AAW.

Previous national prevention strategies and the Surgeon General's Call to Action have recognized the necessity to, “...promote sexual health and responsible sexual behavior" in the promotion of public health and STI prevention within the US (Department of Health and Human Services (US), 2001, p. 1). Refashioning current prevention strategies to address the disparities in STI faced by AAW requires a more comprehensive approach that addresses the unique factors contributing to risk within this population. Expanding methods designed to reduce risk beyond that of condom distribution and counseling in sexual negotiation, will require consideration of several factors that influence behavior using a lens that concentrates on improving sexual health. Thus, holistic approaches that promote sexual health and reduce risky sexual behavior could contribute to better overall outcomes for AAW.

\section{Purpose of the Study}

Recent research has acknowledged positive influences that sexual health approaches may have on behavior with the potential to have a significant role in primary prevention (Hensel \& Fortenberry, 2013). Many preventive efforts to date have focused on encouraging abstinence and reducing risk by developing interventions and disseminating education targeting modes of disease transmission and unintended pregnancy. Using these strategies has yielded evidence-based behavioral and social interventions that have successfully reduced STI, HIV infection, and teen birth rates among certain groups. However, the effectiveness of these interventions has not been observed for AAW. Recently, researchers' efforts have focused on identifying factors responsible for the poor translation of STI education and similar strategies to reduce infection rates and teen or unintended births in AAW. The proposed study will help identify factors that influence the sexual health of AAW. In addition, focus will be placed on recognizing how these factors influence women's participation in sexual behaviors that increase risk of STIs, including HIV and unintended pregnancy. Using this holistic approach will be helpful in understanding the role of sexual health in improving public health efforts directed toward prevention of STI and other negative health outcomes associated with sexual health for AAW. This study has three purposes. The first purpose is to describe the associations between selected emotional and relational factors derived from the Sexual Health Model (SHM) (Robinson et al., 2002) and sexual health in an adult population of AAW recruited from community settings. The second purpose is to examine the relationship among these same factors and sexual risk behaviors in this population. In addition, perceptions of AAWs sexual health will be explored in a subgroup of study participants. The emotional and relational factors of interest, derived from Robinson et al.'s (2002) Sexual Health Model, are detailed in the specific aims and research questions that follow. 


\section{Aims and Research Questions}

There are five specific aims to be addressed within this study.

Specific aim one is to determine the associations between spirituality/religiosity and (a) sexual health in AAW and (b) sexual risk behavior in AAW.

Specific aim two is to determine the associations between depressive symptoms and (a) sexual health in AAW and (b) sexual risk behavior in AAW.

Specific aim three is to determine the associations between gender inequality and (a) sexual health in AAW and (b) sexual risk behavior in AAW.

Specific aim four is to determine the extent to which spirituality/religiosity, depressive symptoms, and gender inequality influence (a) sexual health in AAW and (b) sexual risk behavior in AAW.

Specific aim five is to examine a variety of perceptions of sexual health and was explored using three questions. Question one is, "What are AAW (of varying age, relationship status and reproductive health status) perceptions of sexual health?" Question two is, "What are AAWs (of varying age, relationship status and reproductive health status) perceptions of factors that influence their sexual health?" Question three is "How do AAW (of varying age, relationship status and reproductive health status) perceive the influence of sexual health on their participation in risky sexual behavior?"

\section{Conceptual Model}

Often in nursing and other health professions, conceptual models are used to guide the development of theory, the research design, and interventions used to address health problems (Brathwaite, 2002; Fawcett, 2005). A conceptual model is often considered a broad representation or interpretation of phenomena related to a common theme that is assumed to have some significance (Polit \& Beck, 2012). Using a distinct model to address identified health problems may provide a focused theoretical perspective and practical insight in the development of appropriate nursing interventions or other health services (Fawcett, 2005). This study will use a model that includes abstract concepts that are related to sexual health. Using a model developed with the aim of improving overall sexual well-being could help to identify components that will positively influence outcomes and reduce sexual risk (Robinson et al., 2002). Behavioral change theories and models are often used in HIV and STI prevention research (Robinson et al., 2002; J. Williams et al., 2010). This study is unique in that it will use the SHM to gain insights into the factors associated with sexual health among AAW (Centers for Disease Control and Prevention, 2014e; Robinson et al., 2002).

The Sexual Health Model (SHM) provides a framework through which investigators can successfully address health and sexual behavior by focusing exclusively 
on sexual health and sexuality (Robinson et al., 2002; Satcher, 2013). The SHM acknowledges ten components that influence sexual health promotion and sexual risk reduction (Robinson et al., 2002). Grounded in a comprehensive definition of sexual health, this model identifies specific sexual factors such as sexual self-efficacy and the sexual aspects of intimate relationships which are assumed to contribute to participation in risk behaviors. By placing emphasis on the sexual factors involved in relationships, the SHM addresses the importance of sexual intimacy, identity, and function in combination with emotional and relational factors to better address the many factors that influence STI and HIV risks or risks for other health problems. Addressing the emotional and relational context of sexual health places an explicit focus on factors involved in self-affirmation (e.g., spirituality, religiosity, and depressive symptomology) and sexual negotiation (e.g., gender inequality). Identifying this combination of factors promotes a complete view of sexual health while meeting health prevention goals related to sexual behavior (Robinson et al., 2002).

Having a well-defined perception of sexual health is essential to the use of the SHM (Robinson et al., 2002). By incorporating components of definitions previously established in the literature, Robinson et al.(2002) define sexual health as the way in which individuals demonstrate their knowledge, understanding, and tolerance of sexuality through behavior, emotion, and personal ideals. This state of health is demonstrated through an individual's personality traits or components of individual character and is congruent with diverse components common to an individual's sexual culture.

Encompassing self-acceptance, self-esteem, and personal experience, sexual health is also comprised of the absence of sexual dysfunction, disease, violence, and oppression (Robinson et al., 2002). The authors' definition of sexual health acknowledges the presence of mental health as necessary to establishing congruence between an individual's sexual values and behaviors. Additionally, cultural diversity is an integral component of sexual health because differing social and cultural contexts may cause some variation in definitions of the concept (Robinson et al., 2002).

Stemming from the concept of sexual pluralism, a perspective described by Reiss (1991) as a moral view that accepts multiple ways for individuals to live or make decisions, the SHM identifies culture as influential in making informed choices about health behavior (Robinson et al., 2002). By including culture as an essential component of sexual health, the model incorporates community norms and social experiences as the origins of sexual health (Robinson et al., 2002). The need to consider cultural influences on sexual decision making and risk behavior has been cited as necessary to effectively address health change in ethnic minority populations (J. Williams et al., 2010; Wyatt, Williams, \& Malebranche, 2012). This position is based on the premise that effectiveness in prevention will be improved if culturally specific interventions are implemented with ethnic minority participants. Tailoring preventive efforts to meet the unique needs of targeted populations will more likely evoke adherence and yield positive health outcomes (Robinson et al., 2002). 
Essential components comprising the SHM are holistic and depicted as spokes on the wheel of sexual health (Figure 1-1). Components identified and used by Robinson et al. (2002) to address sexual health include: 1) talking about sex, 2) culture and sexual identity, 3) sexual anatomy and functioning, 4) sexual health care and safer sex, 4) challenges to sexual health, 5) body image, 6) masturbation and fantasy, 7) positive sexuality, 8) intimacy and relationships, 9) positive sexuality, and 10) spirituality. The use of the wheel acknowledges that the relationships among these factors are not viewed as hierarchical, though the nature of these relationships has not been specifically defined (Robinson et al., 2002). Each spoke in the model is assumed to have uniform significance in its overall impact on sexual health. The authors also suggest that the wheel design allows removal of spokes as necessary by future researchers who discover that a spoke does not apply to specific populations (Robinson et al., 2002).

Assumptions included in Robinson and colleague's (2002) definition of sexual health include a balance between an individual's ethical, spiritual, and moral beliefs and their sexual behaviors or standards. According to the SHM, some cultural traditions that are spiritual in nature, may provide an affirming or positive view of sexuality (Robinson et al., 2002). For example, Native-American storytelling uses traditional stories and legends to relay expectations for lifestyle choices and personal accountability. Within Native-American communities, cultural awareness is often used to instill principles that guide behavior and foster perseverance (National Native American AIDS Prevention Center, 2014).

Similar to Native-American tradition, cultural practices involving religion have been found to have a positive impact on perspectives about and expression of sexuality among African Americans. Practices for young AAW that involve religious affiliations and involvement within the church have been associated with conservative attitudes toward sexual activity and decreased participation in sexual risk behaviors (Aalsma et al., 2013). The spirituality spoke on the wheel of sexual health (Figure 1-1) explores the connection between spiritual beliefs and sexual qualities and influences sexual self-care. While Robinson et al. (2002) acknowledge that the concept of spirituality does not require association with a formal religion, both spirituality and religiosity were explored in the current study as culturally-relevant emotional factors that may be associated with sexual health and risk behavior in AAW. Both factors are also geographically relevant when examining the religious landscape of the region where the study will take place. Women participating in this study were recruited from a metropolitan area in the southeastern United States. The southeastern US is a region often referred to as America's "bible belt" due to having a large population of individuals affiliated with formal religions that are predominantly Protestant in denomination (Pew Research Center, 2015).

Circumstances involving chemical dependence, dysfunctional sexual behavior, and sexual violence can pose challenges to achieving an optimal state of sexual health. An individual's emotional capacity can have great bearing on feelings experienced while engaging in sexual behaviors and intimate relationships (Robinson et al., 2002). Experiencing pleasure from sexual encounters or feeling a sense of satisfaction from 


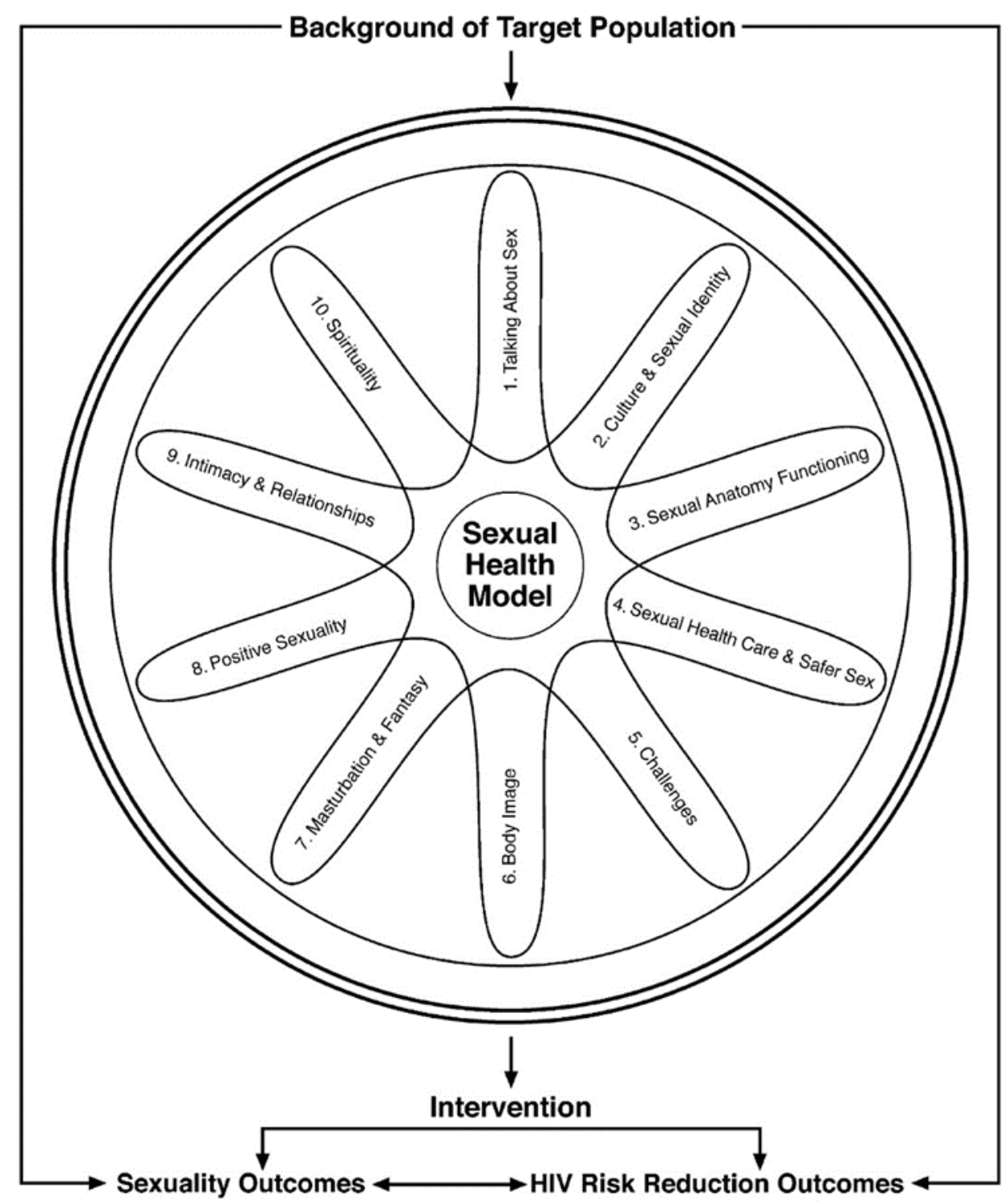

Figure 1-1. The Sexual Health Model.

Reprinted with permission: Robinson, B. B., Bockting, W. O., Rosser, B. R., Miner, M., \& Coleman, E. (2002). The Sexual Health Model: Application of a sexological approach to HIV prevention. Health Education Research, 17(1), 43-57. 
maintaining a personal connection with a partner can evoke emotional responses that may be influenced by an individual's state of mental health. Specifically, conditions that are psychological in nature such as anxiety and depressive disorders may act as barriers to sexual health. The connection between conditions that disrupt mental health and participation in practices that lead to poor health outcomes should be explored and considered as challenges to reaching or maintaining a state of sexual health within African American populations.

The presence of depressive symptoms has been cited as both a predictor and moderator of participation in sexual risk behavior in AAW (Lennon et al., 2012; Seth et al., 2011). Sexual risk behavior, cited in these studies as failing to use condoms during recent sexual encounters, having multiple sexual partners, and engaging in sexual activity while under the influence of alcohol or drugs was increased in AAW with depressive symptoms (Neblett, Davey-Rothwell, Chander, \& Latkin, 2011; Seth et al., 2011). The documented association between depression and sexual risks supports the evaluation of depression in AAW. This important information may be an essential component of risk reduction and sexual health promotion interventions (DiClemente, 2001; Seth et al., 2011). Depression reflects a specific challenge on the SHM wheel that can be addressed effectively with existing approaches if it is identified and appropriately treated. Eradicating or reducing this challenge could reduce substance abuse and engagement in other harmful behaviors prior to participating in sexual activity (Robinson et al., 2002). Eliminating these symptoms through effective counseling and pharmacological approaches could empower AAW, facilitating the path to psychological and sexual health (Seth et al., 2011). This study investigated depressive symptoms as an emotional factor to determine the impact of these symptoms on sexual health and risk behavior in AAW.

Robinson et al. (2002) acknowledge intimacy and relationships as fundamental concepts that have the ability to affect sexual behavior and decision making. It is through the individual's relationships that intimacy, an essential human need, can be achieved (Robinson et al., 2002). By strengthening decision making ability and bargaining power in relationships, individuals can better attain the desired level of intimacy and do so safely. Sexually transmitted infections, HIV, and teen pregnancy prevention strategies for AAW have focused specifically on increasing bargaining power in heterosexual relationships (Centers for Disease Control and Prevention, 2014f; Robinson et al., 2002). Using this framework, bargaining power may also influence overall sexual health by improving sexual communication and the commitment to negotiate safer sexual practices and behaviors (Robinson et al., 2002).

Investigators have acknowledged that issues related to gender inequality have an intrinsic impact on risk behaviors that include condom use while engaging in physical heterosexual encounters in AAW (Paxton et al., 2013). Gender, in this context, suggests that certain behaviors or roles assigned by society are considered acceptable for men and women (World Health Organization, 2002). The intimacy and relationships spoke on the SHM wheel (Figure 1-1) is concerned with the dynamics between two individuals that drive their behavior and interactions. In this study, gender inequality, a relational factor recognized as power differences between men and women that cause inequities between 
the two, was investigated to determine its impact on risk behavior and overall sexual health (World Health Organization, 2002).

One major assumption essential to implementation of the SHM is that healthy sexual choices are made by individuals who are sexually healthy (Robinson et al., 2002). Decisions to participate in sexual risk behaviors are indicative of several underlying issues that may act collectively to inhibit an individual from reaching or maintaining a state of sexual health (Robinson et al., 2002). This study was guided by an approach to sexual health modeled by Robinson and colleagues. The approach is based on identified needs of AAW, focusing on factors pertinent to these women. By focusing only on spirituality and the presence of depressive symptoms as specific emotional factors, as well as gender inequality as a relational factor, this study will examine factors that have not been simultaneously explored from a sexual health perspective. While the entire model was not tested in this study, exploring key relationships within the SHM will provide insights that could successfully address issues that prevent AAW from meeting national prevention goals targeting improvement of their overall reproductive and sexual health. Clinical application of the SHM, using findings from this study, could encourage a level of sexual literacy and security that will reduce sexual risk behavior and facilitate healthy relationship choices.

\section{Definitions of Major Concepts and General Definitions}

\section{Major Concepts}

Variation can be found within existing literature for definitions of most variables explored in this study. Theoretical and operational definitions are provided for important concepts explored within the current study. The concepts sexual health, sexual risk behavior, spirituality, religiosity, depressive symptomology, and gender inequality are defined.

Sexual health. As the definitions of sexual health evolve, a consistent aspect is that it is not the absence of diseases affecting reproductive health (Douglas \& Fenton, 2013). For the purposes of this study, sexual health reflects the way in which individuals demonstrate their knowledge, understanding, and tolerance of sexuality through behavior, emotion, and personal ideals. This state of health is demonstrated through an individual's personality traits or individual characteristics and is congruent with diverse components common to an individual's sexual culture. Encompassing self-acceptance, self-esteem, and personal experience, Robinson and colleagues define sexual health as the absence of sexual dysfunction, disease, violence, and oppression (Robinson et al., 2002). Sexual health will be operationalized using scores on the Sexual Quality-of-Life - Female (SQOL-F) Questionnaire (Symonds, Boolell, \& Quirk, 2005). This multi-dimensional scale briefly assesses sexual quality of life by examining emotional and relational issues that may be affected by sexual dysfunction (Symonds et al., 2005). 
Sexual risk behavior. Sexual activities known to increase a person's risk of acquiring STIs, HIV/AIDS infection, and teen/unintended pregnancy are characterized as sexual risk behaviors. Such behaviors for the purposes of this study will include the following: having unprotected vaginal or anal sex, participating in unprotected oral sex, substance use, and sharing drug use equipment, trading sexual favors for money or other means of support, concurrent sexual partnerships, and being involved in actual or potential partner violence (Centers for Disease Control and Prevention, 2014f; Whyte, 2005). Sexual risk behavior will be operationalized in the current study using scores on the Sexual Activities and Attitudes Questionnaire (SAAQ). The SAAQ is a self-report instrument used to measure sexual activities and sexual attitudes with subgroupings that consist of risky sexual behavior (Noll, Trickett, \& Putnam, 2003). Sexual activities assessed using the questionnaire include the following: 1) age at first intercourse, 2) birth control efficacy, 3) intercourse partners, 4) HIV-risk behaviors, 5) STIs, 6) pregnancies, and 7) sexual behaviors of peers. Sexual attitudes assessed using the SAAQ include the following: 1) sexual preoccupation, 2) sexual permissiveness, 3) internal and external pressure to engage in sex, 4) negative attitudes toward sex, 5) sexual aversion, and 6) sexual ambivalence (Noll, 2003).

Spirituality. In this study, spirituality refers to an emotional factor reflecting the state in which one addresses their own ethical or moral concerns related to sexual health and behavior. From this perspective, spirituality may or may not be identified with formal religious typologies (Robinson et al., 2002). Spirituality will be measured in the current study using scores on the Brief Multidimensional Measure of Religiousness/Spirituality (BMMRS). This measure of wide-ranging aspects of both religiousness and spirituality contains 12 domains including: 1) daily spiritual experiences, 2) meaning, 3) values, 4) beliefs, 5) forgiveness, 6) private religious practices, 7) religious/spiritual coping, 8) religious support, 9) religious/spiritual history, 10) commitment, 11) organizational religiousness, and 12) religious preferences (Fetzer Institute \& National Institute on Aging Working Group, 1999).

Religiosity. Religiosity, like spirituality is concerned with one's personal beliefs and values related to sexual behavior. Religiosity is an emotional factor that refers to the manner in which religion or religious constructs affect the way an individual achieves balance between their ethical and sexual persona (Robinson et al., 2002). Religiosity will also be measured using the BMMRS by focusing on domains to include: 1) private religious practices, 2) religious/spiritual coping, 3) religious support, 4) religious/spiritual history, 5) organizational religiousness, and 6) religious preferences (Fetzer Institute \& National Institute on Aging Working Group, 1999).

Depressive symptomology. Depression is a commonly occurring condition with periods of illness that may interrupt thought processes, mood, and behavior (National Alliance on Mental Illness, 2015). Symptoms of depression, for the purposes of this study include changes in the following: sleep, appetite, ability to concentrate, level of energy, 
interest, self-esteem, or feelings of guilt (National Alliance on Mental Illness, 2015). Depression symptoms will be quantified using scores on the Center for Epidemiologic Studies Depression Scale Revised (CESD-R). The CESD-R is a twenty-item scale used to screen for symptoms of depression or depressive disorder (Eaton, Muntaner, Smith, Tien, \& Ybarra, 2004; Radloff, 1977). Depressive symptoms are measured in nine groups as defined by the American Psychiatric Association Diagnostic and Statistical Manual, fourth edition (DSM-IV) (Center for Innovative Public Health Research, 2014; Radloff, 1977). The nine groups used to assess depressive symptoms include the following: 1) sadness, 2) loss of interest, 3) appetite, 4) sleep, 5) thinking/concentration, 6) guilt, 7) tired (fatigue), 8) movement, and 9) suicidal ideation (Center for Innovative Public Health Research, 2014; Eaton et al., 2004).

Gender inequality. Definitions of gender suggest that certain behaviors or roles assigned by society are considered acceptable for men and women (World Health Organization, 2002). Gender inequality is a concept thought to moderate difficulty asserting or negotiating condom use and preferences not to use condoms in monogamous relationships (Paxton et al., 2013). In this study, gender inequality, was a relational factor identified as power differences between men and women that cause inequities between the two groups (World Health Organization, 2002). Scores on the Sexual Relationship Power Scale (SRPS) were used to quantify gender inequality in the study sample. The 23item SRPS is used as a measure of relationship power with subscales that include the following: 1) relationship control and 2) decision-making dominance (Pulerwitz, Gortmaker, \& DeJong, 2000).

\section{General Definitions}

African American women. African American is a term used to describe the racial or ethnic origin of individuals from a specific minority population. Though somewhat ambiguous in its description, the term is most often used to characterize people originating from any Black racial group in Africa (Centers for Disease Control and Prevention, 2014d). African American women included in this study were those who considered themselves to be Black, African American, or Negro when asked to classify their racial and/or ethnic origin. These women could be from, but not limited to African, American, Caribbean, or West Indian descent.

Reproductive health. Reproductive health focuses on the reproductive system, its processes, and function throughout the lifespan. Achieving this state of health assumes that an individual has the ability to have a responsible sex life, with the absence of disease and with the ability to reproduce. Having adequate access to reproductive healthcare and the right to affordable methods of birth control are also essential components of health in this area (World Health Organization, 2010). 


\section{Significance}

Sexual health has been identified as a diverse field of study with multiple dimensions, each with the potential to improve many components of public health (Douglas \& Fenton, 2013). With reproductive health historically serving as a public health priority, taking the more broad sexual health approach may serve to fill gaps that existing approaches fail to address, thus resulting in negative health outcomes. Using the SHM in this study to identify relationships among specific emotional and relational factors affecting AAW and sexual health and risk behavior can significantly inform nursing practice (e.g., how care is delivered), theory (e.g., how sexual risk behavior are understood), education (e.g., how future nurses are taught and trained), and future research (e.g. intervention development).

Recommendations made in existing literature highlight the need for innovative shifts in the theoretical approach taken toward women's health (J. Williams et al., 2010). These are suggestions supporting the use of the SHM to better understand women's health. This study is theoretically important for two reasons. First, the current study proposes a middle-range theory derived from the SHM to determine its utility and suitability. Second, the study tests selected relationships among important concepts within the SHM that, to date have not been adequately examined. Because the proposed relationships between variables in the model have not been confirmed, this study includes a unique and untested theoretical perspective that could provide important insights. Knowing better the factors that contribute to sexual health and reduce sexual risk among AAW addresses an important gap in knowledge because current models have not been sufficiently effective in addressing this gap. Thus, if the proposed relationships are determined important for this population, study findings could inform development of culturally-relevant evidence and theory-based programs that alleviate depression symptoms and empower women to make healthy choices that are consistent with their spiritual/religious beliefs. To date, existing programs do not simultaneously address these important issues. In addition, addressing these issues in this way potentially reduces the stigma associated with sexuality, and may also improve sexual function, awareness, and relationships for AAW. Figure 1-2 depicts adaptation of the SHM to exclusively include variables examined in this study.

\section{Assumptions}

The following assumptions informed development of the study's conceptual model, research design, and interpretation of its findings.

1. African American women are conscious of their sexual health history and have the ability to provide an accurate account their sexual health and risk behavior. Specific instruments will be used to measure components of each. 


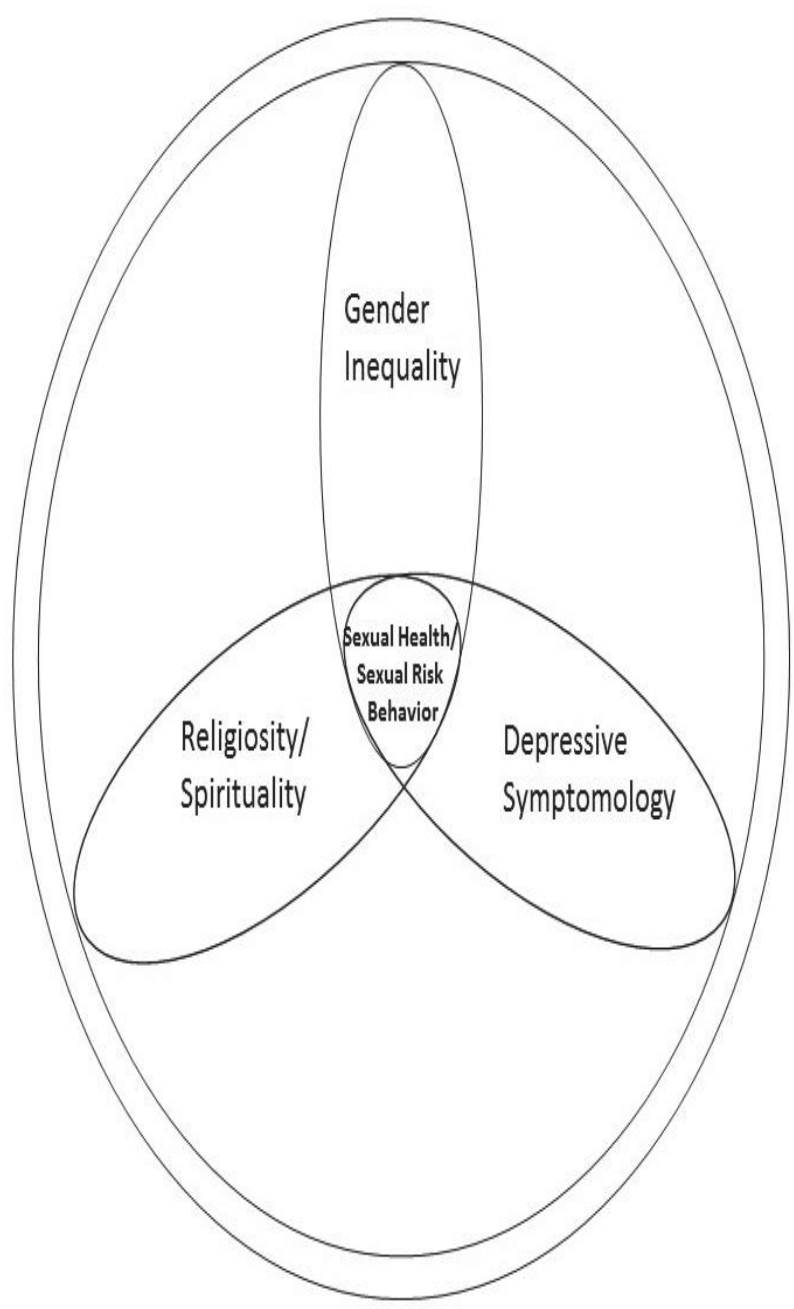

Figure 1-2. Middle Range Theory Derived from the Sexual Health Model.

Adapted with permission: Robinson, B. B., Bockting, W. O., Rosser, B. R., Miner, M., \& Coleman, E. (2002). The Sexual Health Model: application of a sexological approach to HIV prevention. Health Education Research, 17(1), 43-57. 
2. Sexual literacy, comfort, and competence allow AAW to reduce risk through sexual behaviors and relationships (Robinson et al., 2002).

3. Sexual health may be achieved through the removal of spokes present within the SHM as originally developed. Specific spokes identified as pertinent to AAW will be explored to determine their influence on sexual health and risk behavior.

4. Sexual health requires sexual pluralism, which involves accepting others' choices and beliefs in the way in which individuals achieve a moral life (Robinson et al., 2002).

5. Sexually healthy persons are more likely to make sexually healthy decisions (Robinson et al., 2002).

6. Concepts identified within this study's conceptual model are relevant to AAW.

7. Instruments used in this study have demonstrated reliability and validity in previous studies conducted in AAW and will likely be psychometrically sound in the study population.

\section{Limitations}

The following limitations for this study were identified.

1. The SHM, in its preliminary form, serves as a grand theory composed of broad concepts that has not been widely used in practice or research. However, grand theories may be narrowed and can be tested using middle-range theories derived from grand theories (Fawcett, 2005).

2. Using a convenience sample of AAW from a largely urban setting may not yield results that adequately reflect the entire population of AAW in the US (Hulley et al., 2007; Polit \& Beck, 2012).

3. AAW with severe depressive symptoms are less likely to participate in this study, possibly causing nonresponse bias (Polit \& Beck, 2012). Thus, the generalizability of the study findings will be limited to women who are similar to the study sample relative to depressive symptom levels.

4. AAW who participate in behaviors that significantly place them at risk may choose not to participate in this study, limiting the external validity of study findings.

5. During focus group interviews, participants may provide responses in a group setting that are more socially acceptable or politically correct, which may limit the dependability of data obtained using this method (Green \& Thorogood, 2009; Polit \& Beck, 2012). Study participants may be reluctant to fully divulge risky sexual behaviors for fear of appearing promiscuous or unethical. 


\section{CHAPTER 2. REVIEW OF LITERATURE}

\section{Introduction}

Current strategies employed to improve the health of women often give priority to conditions of the reproductive system. This approach to women's health has concentrated largely on contraception (birth control), infertility, menopause, gynecologic cancers, and sexually transmitted infections (STIs) (Centers for Disease Control and Prevention, 2014f). Reproductive health initiatives are designed to protect and maintain the reproductive system during women's childbearing years, an approach known as preconception health (Department of Health and Human Services (US), 2001). Strategies designed to meet women's preconception health needs most often involve the promotion of abstinence, consistent condom use, and regular screening to detect the presence of STIs. The success of these initiatives has traditionally been measured by examining risky behaviors and adverse reproductive health outcomes (Douglas \& Fenton, 2013).

Recently, sexual health, which has a broader health promotion focus, has begun to emerge as an alternative approach in both the science and practice of public health because of its potential to improve negative health outcomes related to sexual behavior (Douglas \& Fenton, 2013; Hensel \& Fortenberry, 2013). Sexual health is a multidimensional state of well-being that involves physical, social, emotional, and relational aspects that reflect an individual's knowledge, understanding, and acceptance of sexuality throughout their lifespan (American Sexual Health Association, 2015; Robinson et al., 2002; World Health Organization, 2002). Despite the efforts of some health care providers to focus holistically on sexual health rather than reproductive health, the burden of conditions such as STIs, human immunodeficiency virus (HIV), and unintended pregnancy remain focal-points of preventive health care due to their disproportionate effects on the health of African American women (AAW). Most evidence-based prevention strategies used to address disparities in AAW's health fail to incorporate the multiple dimensions known to influence sexual health. Persistent trends in reproductive health disparities that include increased rates of HIV infection, unintended pregnancy, and abortion rates reveal the need to shift away from approaches that focus narrowly on sexual risk and to increase the use of approaches anchored in the more comprehensive concept of sexual health (Douglas \& Fenton, 2013; Satcher, 2013).

Data from 2014 indicate that AAW were infected at higher rates with gonorrhea (16.2 times greater), chlamydia (7 times greater), and syphilis (21 times greater) than white women (Centers for Disease Control and Prevention, 2014f). Likewise, AAW continue to represent the highest proportion of new HIV infections (64\%) spread through heterosexual contact (Centers for Disease Control and Prevention, 2014b, 2014c). Consequently, AAW are 20 times more likely than their white counterparts and 5 times more likely than Hispanic/Latina women to become infected with the disease (Centers for Disease Control and Prevention, 2014c). Disparities experienced by AAW are not limited to STI rates. This group is also more likely to experience births that were unplanned or undesired and increasingly report being victims of sexual violence when compared to 
white women (Mosher, Jones, \& Abma, 2012). These and other reproductive health disparities stem from a variety of social, environmental, and emotional factors not specifically accounted for when using traditional approaches to prevent these conditions (Centers for Disease Control and Prevention, 2014e; Douglas, 2011). The World Health Organization $(2002,2010,2015)$ recommends refashioning current women's health prevention efforts to embrace sexuality, wellness, and positive relationships in an effort to improve rates of illegal abortion, infertility, and sexual violence experienced within this population.

Little evidence suggests that current evidence-based, risk-focused interventions lead to long-term sustainability of behaviors that reduce sexual risk and transmission of STIs for AAW (Alexander, Jemmott, Teitelman, \& D'Antonio, 2014; Centers for Disease Control and Prevention, 2014a). Sexual risk behaviors unique to AAW have been shown to increase the risk of acquiring STIs and contribute to the negative health outcomes affecting AAW (McLellan-Lemal et al., 2012; Neblett et al., 2011; Pfileger, Cook, Niccolai, \& Connell, 2013). In addition to behavioral risks, other factors identified as contributing to disparate rates of STI infection and unintended pregnancy in this group are largely emotional and relational in nature. Emotional factors recognized as having a role in achieving the state of well-being required to sustain healthy sexual relationships include depression, stress, anxiety, and self-esteem (American Society for Reproductive Medicine, 2016; Robinson et al., 2002). Additionally, research suggests that relational factors involving the composition of women's sexual relationships and social networks contribute significantly their outcomes. Associations have linked both emotional and relational factors to risky sexual behavior due to their joint effect on women's ability to negotiate within their sexual relationships (Noar et al., 2012; Robinson et al., 2002). Due to this fact, several studies highlight the need to address social and relational factors unique to $\mathrm{AAW}$ as an important aspect of sexual health promotion and risk reduction interventions.

The purpose of this literature review is to synthesize the current science on the sexual health and risk behaviors influencing the well-being of AAW. This review will use components of the Sexual Health Model (SHM) as an organizing framework to examine empirical and practical approaches taken by both researchers and health care providers to address AAW's sexual behavior (Robinson et al., 2002). By integrating a conceptual model that addresses multiple components essential to sexual health, this review will add to current literature by acknowledging factors that move beyond those that are more traditional or behavioral in nature. This review will also build upon current literature by identifying existing gaps and making recommendations for the future of sexual health research.

\section{Methods}

A search was conducted using the CINAHL, PubMed, and Google Scholar databases. Articles included in the review met the following criteria: a) peer-reviewed journal publication; b) primary quantitative, qualitative, or mixed-methods research 
study; c) published between January 2010 and December 2015; and d) examined a sexual, emotional, or relational dimension associated with sexual health as defined by the SHM. For this review, the focus was placed on sexual health promotion, sexual risk and protective behaviors distinct to AAW residing within the U.S. The search also excluded studies examining reproductive health matters such as infertility, Cesarean (C-section) rates, or lower genital tract cancers (uterine, cervical, vulvar, etc.) due to its pointed focus on reproductive health as opposed to the more comprehensive approach of sexual health.

Initially, a combination of keywords including: "sexual health," "sexual risk," "sexual behavior," "sexual practices," "Black women" and "African American women" were used to conduct the search. The combinations of these broad terms yielded 1,951 potential articles of interest. Search terms were then narrowed to also include: "sexual health promotion," "sexual risk behavior," "HIV risk behavior," and "sexual risk reduction," thus reducing the number of potential articles to 1,506. After screening article titles and abstracts to ensure eligibility based on the stated inclusion and exclusion criteria, the number of potential articles decreased in number to 42. An additional evaluation was conducted on remaining articles to exclude duplicates, those focusing exclusively on adolescent African American females, or otherwise not meeting review criteria. This evaluation excluded 28 articles, leaving 14 articles to be included in this review.

\section{Results}

A variety of approaches were employed in the fourteen articles included within this review. Quantitative methodology was primarily used for research studies with sample sizes ranging from 80 to 715 women. The remaining qualitative studies were explorative in nature and included samples of between 10 and 51 women. Cross-sectional designs were used for both quantitative and qualitative studies. Slightly over half of the studies $(\mathrm{n}=8)$ included in this review used a theoretical model or conceptual framework to guide research. Of the studies that employed use of a theoretical model or framework, only one had a conceptual foundation grounded in sexual health. Many studies $(\mathrm{n}=4)$ used the Theory of Gender and Power (Connell, 1987; Jacobs \& Kane, 2011; Wingood, Scd, \& DiClemente, 2000) to acknowledge relational factors that influence AAWs sexual health. Other theories used to guide the research or interventions used to address sexual health include the Theory of Planned Behavior (Ajzen, 1991), reference group theory (Merton \& Rossi, 1950), and the Assessment, Decision, Administration, Production, Topical Experts, Integration, Training, Testing (ADAPT-ITT) model (Wingood \& DiClemente, 2008). Studies included in this review were examined from a holistic perspective and will be discussed according to emotional and relational factors derived from the Sexual Health Model. Each of the studies included in this review recognized

either an emotional or relational factor known to contribute to sexual health or sexual risk behavior in AAW. 


\section{The Sexual Health Model}

The Sexual Health Model (SHM) provides a framework through which investigators and clinicians can successfully address the health behaviors and risks negatively affecting AAW (Robinson et al., 2002; Satcher, 2013). By focusing exclusively on sexuality and sexual well-being, the SHM acknowledges 10 fundamental components of human sexuality (talking about sex; culture and sexual identity; sexual anatomy and functioning; sexual health care and safer sex; challenges to sexual health; body image; masturbation and fantasy; positive sexuality; intimacy and relationships; positive sexuality; and spirituality). The model emphasizes the multiple dimensions involved in meeting the sexual health promotion needs of various groups (Robinson et al., 2002). To more broadly address sexual health and risk behavior, this model reflects the sexual, emotional, and relational circumstances involved in acquiring the ability to protect against STIs, unintended pregnancy, and sexual violence. The model emphasizes self-affirmation and sexual negotiation as important skills needed to support personal prevention efforts. This literature review examines the current literature through the SHM lens, which informs the search criteria used.

Sexual health. Since its initial acknowledgment and definition by the World Health Organization (WHO) in 1975, the role of sexual health has been refined and continues to progress in both the science and practice of public health (Douglas \& Fenton, 2013; World Health Organization, 1975). During its evolution, sexual health has maintained its fundamental feature distinguishing the concept from similar approaches by focusing on overall well-being. Through its concentration on holistic wellness, sexual health encompasses components that involve: accepting individual sexuality and sexual rights, experiencing sexual pleasure, educating individuals about sexual health, and reducing negative outcomes related to sexual behavior (Douglas \& Fenton, 2013; Robinson et al., 2002; World Health Organization, 2002). Recent approaches taken toward sexual health have begun to recognize both the individual and social determinants involved in achieving this state of well-being (Satcher, 2013; Sharpe et al., 2012; World Health Organization, 2002). In an effort to embrace individual awareness and the cultural or societal impact on sexuality, research and prevention programs have started to address the multiple domains that comprise the concept of sexual health (Douglas \& Fenton, 2013; Robinson et al., 2002).

Most recently, sexual health research and programs or initiatives designed toward the improvement of health outcomes have started to integrate emotional, relational, and sexual factors in an effort to align conceptually with the WHO definition of sexual health (Centers for Disease Control and Prevention, 2014e; Robinson et al., 2002). Similarly, the surveillance and measurement of sexual health is also undergoing a transition to identify indicators that adequately gauge each of the domains encompassed in the more holistic concept. Despite ongoing efforts to migrate away from using more traditional measures, in a review of national surveillance systems and health surveys, Ivankovich, Leichliter, and Douglas found that, most data systems and surveys used in the US tend to focus primarily on the behavioral aspects of sexual health including sexual risk or the 
surveillance of negative health outcomes (2013). Collaborative efforts between individual researchers and the Centers for Disease Control and Prevention have begun to expand the tracking and measurement of sexual health in the US to include pertinent domains involving an individual's level of sexual health education, communication abilities, and sexual approach or attitude (Douglas \& Fenton, 2013; Ivankovich et al., 2013). In addition, social- and community-level indicators have been integrated into national surveillance efforts to include sexual behaviors and relationships, health outcomes, and access or utilization of sexual health services (Douglas \& Fenton, 2013; Ivankovich et al., 2013).

Canada's Public Health Agency has successfully carried out WHO recommendations made to include emotional and relational domains in the tracking and measurement of sexual health (Douglas \& Fenton, 2013; Ivankovich et al., 2013; Smylie et al., 2013). Canadian researchers have created and validated a 75-item survey to measure the multiple domains of sexual health for Canadian young adults (Smylie et al., 2013). The sexual health domains measured in the survey included: physical, emotional, and social well-being related to sexuality; an individual's approach to sexuality; sexual relationships and experiences; and coercion and violence (Smylie et al., 2013). To date, no such survey has been created and/or endorsed by US public health agencies as an accurate measure of the nation's sexual health for various populations or groups.

The inclusion of specific emotional, relational, and sexual components involved in sexual health are known to vary for individuals in different groups (Robinson et al., 2002). The variety of factors influencing the sexual health of groups stems largely from the influence of culture, life experiences, and the social norms accepted by individuals (Robinson et al., 2002; Sharpe et al., 2012). Research indicates that for AAW, culturally congruent models of sexual health and interventions used to address its components should reflect cooperation between individuals, family and community, faith-based organizations, cultural inspiration to survive obstacles, and behaviors derived from accepted stereotypes (J. Williams et al., 2010; Wyatt et al., 2012). Despite these and similar acknowledgements, sexual health research has historically employed learning theories or conceptual frameworks that focus on the way in which information is processed and maintained, rather than integrating models that focus specifically on the multiple dimensions of sexual health. Thus, the use of such theories may neglect specific factors distinct to diverse populations (J. Williams et al., 2010). For AAW using theories not grounded in sexual health has contributed to the development of interventions that have centered on individual level characteristics and place emphasis on the promotion of abstinence, consistent condom use, and regular STI screening (Alexander et al., 2014; Centers for Disease Control and Prevention, 2013, 2014a). The promotion of these and similar interventions in AAW has not translated to sustainable changes in behavior that have led to significant declines in negative health outcomes including STI and unintended or teen pregnancy (Centers for Disease Control and Prevention, 2013; J. Williams et al., 2010).

Recent research efforts designed to improve the sexual health of AAW have started to shift their concentration to emphasize specific emotional and relational factors 
to promote behavior change and reduce sexual risk (Alexander et al., 2014). In a study conducted by Murray et al., taking a theoretical approach grounded in sexual health heightened researchers' understanding of the need to integrate STI and pregnancy prevention with the promotion of healthy sexual behavior within women's relationships and in their communities (2013). Findings from this qualitative study reveal that for young AAW several emotional and relational factors influenced the degree to which they participated in protective behaviors including condom and contraceptive use. Women in this study identified relational factors such as trust and the significance or duration of their relationships as contributing to their decisions to discontinue engaging in protective behaviors (Murray et al., 2013). An emotional factor, intimacy, was also cited as a central component that helped to determine women's participation in condom and/or contraceptive use. Findings from the Murray et al. study contradict those from a previous study conducted in mostly in AAW (70\%), which hypothesized that feelings of intimacy contributed to increased condom use. For these study participants, condom use was linked to the protection of those with whom they felt closest (Damani et al., 2009). Other emotional factors identified in research exploring the sexual health of AAW include: selfimage, self-esteem, depression, and mental health (Bancroft, Long, \& McCabe, 2011; Brown, Webb-Bradley, Cobb, Spaw, \& Aldridge, 2014; Mincey \& Norris, 2014).

In addition to recognizing the impact of emotional and relational factors, researchers have also begun to acknowledge the important impact of social factors on the sexual health of AAW (Douglas \& Fenton, 2013; Sharpe et al., 2012). Research has examined race or ethnic background, the communities in which individuals reside, access to healthcare, economic status, social networks, and communication patterns to determine their influence on AAW's sexual health (Bancroft et al., 2011; Biello, Ickovics, Niccolai, Lin, \& Kershaw, 2013; McLellan-Lemal et al., 2013; Murray et al., 2013; Sharpe et al., 2012). According to Biello and colleagues, black or African American race appeared to be a factor associated with lower ages of sexual initiation (2013). In the previously mentioned Murray et al. study, social factors involving parental and societal influence seemed to shape women's attitudes regarding their relationships and acceptance of the possibility of pregnancy (2013). Other empirical findings reveal that social determinants including financial adversity, relationship status, gender roles, and family or household structure have the potential to manipulate and distinguish the emotional and relational factors that underlie AAWs reproductive and sexual health (McLellan-Lemal et al., 2012; Sharpe et al., 2012).

As the science of sexual health continues to emerge, growing research in this area has provided great insight toward improvement of the practice of public health. The introduction of the holistic concept of sexual health has exposed the level of complexity involved in relationships developed from emotional, relational, social, and/or sexual factors distinct to AAW (World Health Organization, 2002). Additional work is required for sexual health researchers to continue the development of a survey or tool to accurately measure sexual health. The development of such a tool will work to improve the national surveillance and psychometric measurement of the multiple dimensions that influence sexual health (Satcher, 2013). Currently, sexual health research continues to provide opportunities for innovation in public health prevention strategies used to address 
disparities in sexual health. For each of the studies included in this review, a synthesis of sexual, emotional, and relational factors identified as pertinent to AAW will be appraised to determine their potential to improve the overall well-being of AAW.

Sexual risk behavior. As previously mentioned, measures used to gauge sexual health often tend to focus narrowly on behavioral aspects that increase individuals' sexual risk (Douglas \& Fenton, 2013). The identification of patterns of sexual risk behaviors and development of interventions to reduce or eliminate those behaviors has long since been the focus of research studies examining the sexual and reproductive health of AAW (McLellan-Lemal et al., 2012; Neblett et al., 2011; Reid et al., 2014). With heterosexual contact being attributed to most new HIV infections and other STIs in AAW, behavioral factors frequently identified in the examination of sexual risk for these women include: inconsistent condom use, having multiple or concurrent sexual partners, and the use of illicit drugs (Centers for Disease Control and Prevention, 2014b; McLellan-Lemal et al., 2013; Perkins, Stennis, Taylor Spriggs, Kwegyir-Afful, \& Prather, 2014). Research has shown that other behavioral factors associated with AAW's sexual risk include: age of sexual initiation (before 15 years of age) and range of sexual activity (anal, oral, or vaginal sex) (Liu et al., 2015; Neblett et al., 2011; Pfileger et al., 2013).

Many studies investigating the sexual behavior of AAW focus on specific activity that increase risk and contribute to existing disparities in sexual health. For AAW, sexual risk is often measured by the practice of multiple sexual behaviors associated with the transmission of STIs including HIV infection (McLellan-Lemal et al., 2012). In most research studies examining AAW's sexual behavior, consistency in condom use, while varied in its definition, is typically characterized as women's use of condoms during each vaginal or anal sexual encounter occurring over a duration of $60-90$ days (Crosby et al., 2013; DePadilla, Windle, Wingood, Cooper, \& DiClemente, 2011). The consistency of AAW's condom usage has often been described as low or sporadic (Perkins et al., 2014; Pfileger et al., 2013). Additionally, research studies examining AAW's sexual behavior often explore women's condom use in conjunction with having multiple or concurrent sexual partners (Grieb, Davey-Rothwell, \& Latkin, 2012a; McLellan-Lemal et al., 2012; Nunn et al., 2012). Concurrent sexual partnerships involve having sexual encounters with multiple partners that overlap or occur over the same span of time (Nunn et al., 2012). AAW have frequently been identified as being more likely than women of other groups to engage in concurrent partnerships (Adimora, Schoenbach, Taylor, Khan, \& Schwartz, 2011; Nunn et al., 2012). Both inconsistent condom use and concurrent sexual partnerships have been identified as high-risk behaviors engaged in more frequently by AAW and are commonly used to measure AAW's sexual risk (McLellan-Lemal et al., 2012; Neblett et al., 2011; Perkins et al., 2014).

Much like research focusing broadly on sexual health, recent investigations of AAW's sexual risk and behavior have begun to acknowledge that factors contributing to the increased incidence and prevalence of negative health outcomes may be determined by components that extend beyond individual behaviors (Liu et al., 2015; McLellanLemal et al., 2012; Neblett et al., 2011). The inclusion of factors in the examination of 
AAW's sexual risks, that are emotional and relational in nature, have served to expand upon the existing body of knowledge related to sexual behavior (McLellan-Lemal et al., 2013; Sharpe et al., 2012). AAW's sexual relationships have broadened the perspective traditionally taken to address the risk faced by these women (McLellan-Lemal et al., 2012; Sharpe et al., 2012). Nehl and colleagues (2015) reveal that the composition of AAW's sexual relationships and the determination of whether sexual partners were steady or casual moderated women's consistent condom use. Researchers suggest that AAW who have steady sexual partners have decreased odds of consistently using condoms (Nehl et al., 2015). Additionally, having a drug using sexual partner also decreased the odds of consistent condom use for AAW (Nehl et al., 2015). These results indicate that relational factors involving women's relationships or type of sexual partner may influence participation in risky sexual behavior and increase AAW's risk of acquiring STIs or experiencing unintended pregnancy.

Additional relational factors associated with condom use and other risky behaviors in AAW are related to women's ability to communicate and negotiate within their relationships (Crosby et al., 2013). Investigations into the balance of power existing within sexual relationships reveal that some women perceive negotiations of power as a challenge within their relationships (McLellan-Lemal et al., 2013). In a qualitative study conducted by McLellan-Lemal et al. (2013), challenges created by relationship power caused discord within relationships that could lead to sexual violence. Other studies indicate that imbalances in power increased women's sexual risk from being coerced into participation in behaviors including drug use and concurrent sexual partnerships (McLellan-Lemal et al., 2012; Nehl et al., 2015).

Various social factors have also been identified as having a significant influence on AAW's participation in high-risk sexual behaviors. Similar to comparisons made with sexual health, social determinants including AAW's neighborhoods or communities and their economic status are determined to be central to participation in concurrent sexual partnerships (Fichtenberg, Jennings, Glass, \& Ellen, 2010; Neblett et al., 2011). Neblett et al. (2011) conducted a study examining characteristics of AAW's social networks to determine their influence on participation in risky sexual behavior. Researchers found that AAW who had larger social networks, or groups of people with whom they interacted with socially, were more likely to have a sexual partner who engaged in sexual risk behaviors. Thus, this association was considered as increasing women's sexual risk (Neblett et al., 2011). Another study, examining AAW's sexual networks, or groups of individuals with whom women were connected to sexually (e.g., sexual partners), found that financial adversity was associated with having sexual networks that included greater than two people (Fichtenberg et al., 2010). Additionally, the sexual networks of women of lower socioeconomic status included individuals who were more likely to acquire STIs (Fichtenberg et al., 2010).

While the use of behavioral measures fail to address each of the components encompassed in the multidimensional approach of sexual health, studies examining specific behaviors contributing to this state of well-being will also be included this review. Research studies examining AAW's sexual risk or specific risk behaviors will be 
synthesized to determine the impact sexual, emotional, and relational factors have on the sexual health and risk behavior of AAW. Each study included in this review recognized at least one emotional (spirituality/religiosity or depression/depressive symptomology) or relational (gender inequality) factor as a variable contributing to the sexual health and/or risk behavior of AAW.

\section{Emotional Factors}

Spirituality/religiosity. Emotional factors often recognized by researchers as having an influence on the sexual health and risk behavior of AAW include religiosity and/or spirituality. Research has revealed that varying degrees of religiosity or spirituality may improve individuals' perception of their overall health or state of well-being (Fetzer Institute \& National Institute on Aging Working Group, 1999). More specifically, when looking at sexual health, spirituality, which may not include doctrine from formal religions, allows an individual to address their sexuality from a moral or ethical perspective (Robinson et al., 2002). AAW are reported as the racial or ethnic group with the highest level of religious commitment, by being most likely to report a religious affiliation and regularly engaging in formal religious practices (e.g., going to church and praying) (Pew Research Center, 2015; Wingood et al., 2013). For this reason, churches have been sought as an optimal setting to implement sexual health promotion and risk reduction interventions (Stewart, Sommers, \& Brawner, 2013; T. Williams, Pichon, Davey-Rothwell, \& Latkin, 2016; Wingood et al., 2013).

Five studies included in this review examined components of religiosity/spirituality as a factor influencing the sexual health or risk behavior of AAW. In a longitudinal study conducted by Aalsma et al (2013), researchers examined 328 young adult AAW's sexual behavior, sexual conservatism, and religiosity over time. Sexual conservatism was defined in this study as the validation or demonstration of conventional sexual principles such as sexual intercourse before marriage, a variable that was found to be associated with both religiosity and sexual risk behavior (Aalsma et al., 2013). Results from this study reveal decreases in younger women's sexual conservatism and religiosity over a 4-year time period. However, women's religiosity returned back to baseline by the study's 4-year end-point, while sexual risk behavior appeared to progressively increase over time (Aalsma et al., 2013). These results indicate that changes in religiosity predict sexual conservatism, a variable in which decreases will be likely to result in increased participation in sexual risk behaviors (Aalsma et al., 2013). This study provides a unique perspective due to it longitudinal design and reveals the ongoing interaction between young women's religiosity and sexual behavior. This study unveils the long-standing potential that religiosity as an emotional factor may have on the sexual health of AAW.

Other facets of religiosity were addressed in two studies as being culturally congruent variables contributing to the improvement of AAW's sexual health and risk behavior (T. Williams et al., 2016; T. Williams, Pichon, Latkin, \& Davey-Rothwell, 
2014). One research study conducted by Williams, Pichon, Davey-Rothwell, and Latkin (2014) assessed 434 high risk AAW to determine the relationship between having the emotional support of women's church congregation and their participation in behaviors designed to reduce sexual risk and prevent HIV. Study results suggest that AAW who felt loved or listened to by their church congregation or belonged to congregations with ministries or groups that they felt were helpful in addressing their problems experienced greater comfort discussing HIV prevention in church and were more likely to follow-up on HIV testing to obtain results (T. Williams et al., 2014). In a similar study, these researchers investigated the relationship between church attendance and women's discussion of topics related to sexual health. Results from this study reveal that for highrisk AAW, regular church attendance significantly predicted the number of sexual health topics women discussed with their friends and sexual partners (T. Williams et al., 2016). Both studies findings indicate that facets of religiosity may improve AAW's sexual health and reduce sexual risk.

The remaining two studies, both of which recognized religiosity as a pertinent emotional factor associated with AAW's sexual health and risk behavior, evaluated HIV prevention interventions carried out in a church or faith-based setting. With previous research demonstrating the connection between African Americans' commitment to religion and religious practices, many researchers have sought the community influence of faith-based organizations to promote sexual health (Pew Research Center, 2015; Stewart et al., 2013). One study compared the effectiveness of an evidence-based HIV prevention intervention with $\mathrm{P} 4$ for Women, a faith-based adaptation of the same intervention, both designed for AAW (Wingood et al., 2013). Researchers found that both interventions significantly increased women's consistency of condom use (Wingood et al., 2013). Furthermore, P4 for Women, the faith-based alternative, demonstrated additional significant effects in women's behavior that included maintaining longer periods of abstinence, thus indicating success in its implementation (Wingood et al., 2013). In another study, Stewart (2014) examined the barriers and facilitators associated with adapting an HIV prevention intervention to be carried out in churches. This mixedmethods study revealed that both church leaders' and members' perception of the necessity of church involvement in HIV prevention facilitated implementation of the study's intervention (Stewart, 2014). However, the thought that discussion of sexuality should be private within the church served as a barrier. Findings from both studies demonstrate utility in the implementation of faith-based interventions directed toward the reduction of AAW's sexual risk and sexual health promotion (Stewart, 2014).

Depression/depressive symptomology. Most definitions of sexual health include mental wellness as a component essential to achieving a state of well-being related to sexuality (Robinson et al., 2002; World Health Organization, 2002). Another emotional factor frequently recognized in sexual health literature as having the potential to influence AAWs sexual health and behavior is depressive symptomology. Depressive symptomology or the presence of symptoms stemming from depression and other depressive disorders has been noted to cause psychological and emotional distress that may influence women's sexual risk and behavior (Lennon et al., 2012; National Institute 
of Mental Health, 2016). Like STIs and unintended pregnancy, depression and psychological distress among AAW have been recognized as significant public health concerns due to the impact of social determinants including poverty and lack of access to mental health care (Department of Health and Human Services (US), 2001; Lennon et al., 2012).

Five studies included in this review examined the influence of depressive symptomology on the sexual health and risk behavior of AAW. In a longitudinal study conducted by Seth et al. (2011), depressive symptomology was examined as a predictor of sexual risk behavior, sexual communication, and STIs in young adult AAW.

Depressive symptomology was measured in this study using the short (8-item) version of the Center for Epidemiological Studies Depression Scale (CESD), a tool that assesses the presence of symptoms occurring over the last seven days (Melchior, Huba, Brown, \& Reback, 1993). The initial examination of 715 young adults, a sample that was reduced to 605 after 12 months, revealed that after six months' time, the presence of higher levels of depressive symptoms predicted having multiple sexual partners and not using condoms during women's last sexual encounter (Seth et al., 2011). Additionally, at both six and 12-month time points, depressive symptoms predicted engaging in concurrent sexual partnerships, experiencing fear in communicating about condoms with sexual partners, and having sex while under the influence of illicit drugs or alcohol (Seth et al., 2011). For women in this study, the presence of depressive symptoms was not a predictor of STIs (Seth et al., 2011). The results of this study reveal that depressive symptomology, as an emotional factor significantly influenced women's sexual behavior, thus negatively contributing to the multiple dimensions of AAW's sexual health.

A sequential exploratory mixed methods study conducted by Brawner et al. (2012) examined the relationship between clinical depression and sexual behavior in a sample of 128 young adult AAW. Both non-depressed and depressed participants (women reporting a diagnosed depressive disorder or participants screened with high levels of depressive symptoms) completed the study's quantitative phase, while a subsample also completed individual or focus group interviews. Most of the sexual behaviors assessed in this study, vaginal, anal, and oral sexual activity, condom use, and STI testing, were influenced by depression and/or feelings of depression (Brawner et al., 2012). Depressed study participants reported an increased frequency of sexual activity, having multiple sexual partners, and engaging in sexual encounters while under the influence of illicit drugs or alcohol (Brawner et al., 2012). The study's qualitative results corroborated these findings by illustrating young AAW's perception that their feelings of loneliness, which were thought to likely be derived by depression, affected many of their sexual decisions (Brawner et al., 2012). Much like the previous study, this study's findings also demonstrate the impact of depression and depressive symptoms as an emotional factor influencing the sexual health of young AAW.

Two additional studies included in this review both investigate the presence of depressive symptoms as a variable influencing the sexual health or risk behavior of AAW. One study conducted by Mincey and Norris (2014) examined the relationship between emerging adult AAW's sexual health and the presence of depressive symptoms. 
Sexual health was measured in this study by evaluating components of women's behavior including: age of sexual initiation (before age 17), having unprotected sex, and having been diagnosed with a STI (Mincey \& Norris, 2014). Study results indicate that for younger AAW depressive symptoms, measured using the CESD, directly affected participation in behaviors used to evaluate sexual health. In this study, young women (ages 18 -27) with higher levels of depressive symptoms reported increased participation in sexual risk behaviors (Mincey \& Norris, 2014). However, for women between the ages of 25 and 34, a significant relationship was not found between sexual health (participation in sexual risk behaviors) and the presence of depressive symptoms (Mincey \& Norris, 2014). Another study conducted by Grieb, Davey-Rothwell, and Latkin (2012b) investigated depressive symptomology as a correlate of concurrent sexual partnerships in AAW. Study results reveal that while a large proportion of the study's entire sample $(\mathrm{N}=337)$ reported experiencing depressive symptoms, women in concurrent partnerships reported having higher levels of depressive symptoms (indicated by a CESD score $\geq 16$ ) (Grieb et al., 2012b). Results from both studies demonstrate a significant influence of depressive symptoms on AAW's sexual health and risk behavior.

The remaining study included in this review evaluated the effectiveness of a cognitive/behavioral intervention designed to address sexual risk behavior and reinfection of STIs in both Hispanic and AAW (Holden et al., 2008). Sexual risk behaviors were characterized in this study as: "non-mutual monogamy" (having a sexual partner who is not monogamous or one that has multiple sexual partners), unprotected sex, douching after sexual intercourse, and rapid assumption of new sexual partners (less than 3 months) (Holden et al., 2008). In a sample of 477 women, that included both depressed and nondepressed women, use of an evidence-based risk reduction intervention demonstrated lower levels of sexual risk and reinfection rates for STIs at 1-year follow-up (Holden et al., 2008). Despite initially having greater levels of sexual risk, both moderately and severely depressed women who received the intervention, demonstrated a sustained reduction in risk and reinfection of STIs when compared to controls (Holden et al., 2008). Results from this study highlight the potential of integrating emotional factors in interventions used to improve sexual health by reducing risk.

\section{Relational Factors and Gender Inequality}

Factors surrounding intimate or sexual relationships have provided insight into women's sexual risk and behavior. For AAW, issues related to assumed gender roles or inequities related to gender are thought to negatively impact women's ability to communicate and negotiate within their intimate relationships. Recently, the influence of power and gender within women's relationships has been recognized as a factor influencing negative health outcomes and sexual health disparities in AAW (Blanc, 2001). This review includes four research studies investigating the influence of gender inequality on AAW's sexual risk and behavior (McLellan-Lemal et al., 2013; Nehl et al., 2015). 
One study examined how specific constructs from the Theory of Gender and Power predicted condom use among a sample of 701 young AAW (DePadilla et al., 2011). Researchers tested a model generated from the Theory of Gender and Power by applying its three major social structures to examine HIV risk behaviors in a sample of AAW: sexual division of labor, structure of affective attachments and social norms, and sexual division of power (DePadilla et al., 2011). The model was developed using structural equations. A secondary data analysis was conducted to validate the model. Study results identified self-efficacy in partner communication as the strongest predictor of condom use in young AAW (DePadilla et al., 2011). This study's findings reveal that relational factors are both theoretically and clinically important factors involved in AAW's sexual health and risk behavior.

A study conducted by Raiford, Seth, and DiClemente (2013) assessed the associations among relationship imperative, sexual risk behavior, and associated psychosocial risk factors. In this study, relationship imperative was viewed as young AAW's perception of an intimate relationship as imperative to assume or maintain a positive self-concept (Raiford et al., 2013). Researchers measured relationship power, sexual risk behavior, and the presence of sexually transmitted infections in a sample of 715 young adult AAW (Raiford et al., 2013). This study's results indicate that women who felt a stronger sense of identity within their relationships or "endorsed a relationship imperative" were more likely to 1) engage in unprotected sex, 2) experience decreased relationship power, and 3) not feel confident in their ability to decline sex, range of sexual activity, sex while a partner was under the influence of illicit drugs or alcohol, or abuse from their sexual partners (Raiford et al., 2013). Additionally, young AAW who experienced less power within their relationships, recent abuse by partners, and felt an inability to deny sex were more likely to be infected with chlamydia, gonorrhea, or trichomonas (Raiford et al., 2013).

The two remaining studies included in this review explore gender inequalities related to the negotiation of power within relationships and their influence on women's sexual behavior (McLellan-Lemal et al., 2013; Nehl et al., 2015). In a qualitative study conducted by McLellan-Lemal et al (2013), researchers explored AAW's perceptions regarding relationships, relationship power, and their ability to make decisions. Two themes emerged from women's discussion of their relationships. One theme focused on the contradiction between women's relationship desires and their real-life relationships, while the other theme concentrated on women's perception of challenges within their relationship. One relationship challenge was identified as the negotiation of relationship power. Results from this study illustrate the fact that women's relationship ideals embodied an acceptance of infidelity and use of sex as a mode of relationship formation and sustainability. Women did not however appear to have standards regarding their sexual risk (McLellan-Lemal et al., 2013). An additional study conducted by ManfrinLedet, Porche, and Westbrook (2015) examined the relationship between intimate partner violence, HIV risk behavior, and decreased relationship power in AAW. Study researchers also used the Theory of Gender and Power to investigate130 AAW of childbearing age. For women who experienced intimate partner violence, study results reveal significant relationships between intimate partner violence and decreased power 
within their relationships (Manfrin-Ledet et al., 2015). Study participants who reported fearing their current or previous partners also reported decreased relationship power (Manfrin-Ledet et al., 2015). Each of these studies reveals the importance of addressing inequities in power as a relational factor in the examination and improvement of AAW's sexual health and risk behavior.

\section{Conclusion}

This review of literature appraised the sexual health and risk behavior of AAW from a perspective grounded in the WHO definition of sexual health. Use of the SHM fills an existing gap in both sexual health research and clinical practice by revealing patterns in AAW's behavior that contribute to the multidimensional state of well-being related to sexuality. This appraisal of current literature collectively addresses emotional and relational factors known to influence AAW's sexual health that, to my knowledge, have not been concurrently reviewed in existing literature. While each of the studies included in this review do not holistically address sexual health, all studies address important facets of women's behavior that have been recognized as increasing AAW's risk of acquiring STIs or experiencing unintended pregnancy. Findings from this review identify specific factors pertinent to addressing the sexual health of AAW which reflect African American culture, life experiences, and accepted social norms. 


\section{CHAPTER 3. METHODOLOGY}

This chapter includes a description of the methodology and research design used to conduct this study. An explicit explanation of the study's sample, setting, instrumentation, and procedures used during data collection, analyses, and in the protection of human subjects is provided.

\section{Study Design}

This study employed a cross-sectional, descriptive, correlational design to identify factors that influence sexual health and sexual behaviors hypothesized to increase risks for negative health outcomes such as STIs and unintended pregnancy, among other conditions in African American women (AAW). A parallel or concurrent mixed methods design was employed, whereby qualitative components were integrated with quantitative methods and analyses to enhance and support study findings. Quantitative data were obtained to examine relationships among selected emotional factors (i.e., spirituality, religiosity, and depressive symptomatology) and a relational factor (i.e., gender inequality) and both sexual health and risk behaviors in an adult population of AAW recruited from community settings. A qualitative (QUAL) strand, which included focus group interviews, was conducted concurrently with the study's quantitative (QUAN) component, for a mixed methods approach. The qualitative component was used to explore women's perceptions of sexual health in a subgroup of study participants to enrich data derived from a quantitative perspective. Interpretive integration was completed following the analysis of both qualitative and quantitative data to merge study findings and draw inferences from the combination of approaches used.

\section{Sample and Setting}

This study targeted AAW residing within Memphis metropolitan area communities to identify factors influencing their sexual health and risk behaviors. Flyers, posters, email listservs, and participant referrals were used to recruit the convenience sample from various sources within the community. Flyers and posters were distributed throughout the University of Tennessee Health Science Center (UTHSC) campus and among members of various community organizations. Organizations including African American community service sororities, social clubs, and other formal groups were contacted by the principal investigator to petition members for study participation and to disseminate informational flyers. UTHSC faculty and student listservs were used to deliver email communication seeking participants from eligible subscribers. Snowball sampling was also used as enrolled participants identified and referred individuals for potential study participation. Recruitment goals included obtaining an adequate sample of AAW varying in age and demographic background to ensure generalizability of study findings. Study enrollment, which involved completing the study's QUAN strand, was 
extended to all interested individuals who met inclusion/exclusion criteria as indicated below.

Purposive sampling was used to recruit a subsample of enrolled study participants to complete the study's QUAL component. The study's QUAL sample was conveniently selected based on specific criteria to provide variety in women's age, relationship status, and reproductive health status.

\section{Inclusion Criteria}

The criteria for study inclusion consisted of the following:

1. $\quad$ self-identified AA female

2. reported sexual activity within the past 12 months (1 year)

3. Memphis metropolitan area resident to include the following counties: Shelby (TN), Crittenden (AR), St. Francis (AR), Desoto (MS), Tunica (MS), Marshall (MS), Tipton (TN), and Fayette (TN)

4. at least 18 years of age or older at the time of enrollment

5. ability to read, speak, and understand English.

\section{Exclusion Criteria}

The criteria for study exclusion consisted of the following:

1. no reported sexual activity within the past 12 months (1 year);

2. actively seeking/attempting to become pregnant;

3. residing outside of the Memphis metropolitan area;

4. younger than 18 years of age at the time of enrollment;

5. unable to read, speak, or understand English.

\section{Sample Description}

The entire study sample was comprised of 50 conveniently selected, selfidentified African American female residents of the Memphis metropolitan area. Snowball sampling was used to generate most of the community-based sample by having women who were previously enrolled in the study seek interest from additional women with whom they were familiar. Recruitment flyers and emails distributed to students, employees, and patrons of the University of Tennessee Health Science Center were used to obtain the remainder of the study sample.

The study's QUAN sample, which reflects the full study sample, was largely heterosexual (98\%) and reported mostly (54\%) being single (44\%) or divorced (10\%). QUAN study participants had a mean age of 38.7 with a range of 21 to 64 years. 
Similarly, the study's QUAL sample $(\mathrm{n}=19)$, a subset of the full study sample, was primarily heterosexual (94.7\%) with a comparable makeup regarding their age and relationship status. QUAL study participants ranged in age from 25 to 64 years with a mean age of 41.1 years. The QUAL sample was predominately married (42\%) or living with their partner (11\%). Much like the study's QUAN sample, nearly half (47\%) of these women reported being unmarried with slightly over $10 \%$ reported being divorced, with $36.9 \%$ being single.

\section{Instrumentation}

Six paper and pencil instruments were used to quantify emotional and relational factors being examined in this study. Instruments used to collect demographic data from study participants and measure sexual health, sexual risk behavior, religiosity, spirituality, depressive symptomology, and gender inequality included the following: Participant Demographic Data Form, Sexual Quality of Life - Female (SQOL-F) Questionnaire (Symonds et al., 2005), Sexual Activities and Attitudes Questionnaire (SAAQ) (Noll et al., 2003), Brief Multidimensional Measure of Religiousness/Spirituality (BMMRS) (Fetzer Institute \& National Institute on Aging Working Group, 1999), Center for Epidemiologic Studies Depression Scale Revised (CESD-R) (Eaton et al., 2004), and the Sexual Relationship Power Scale (SRPS) (Pulerwitz et al., 2000). Focus group interviews with structured interview items were also used to gather data from participants' perspective on factors influencing their sexual health.

\section{Participant Demographic Data Form}

An investigator-developed questionnaire was used to gather pertinent demographic information from study participants related to sexual health and risk behavior. The self-report questionnaire contained distinguishing details including the following: age (in years), relationship status (married/in a stable relationship; not married), sexual orientation (heterosexual; gay/lesbian; bisexual), educational level (years of education), employment status (employed; not employed), health insurance coverage (private/employer provided; government provided [i.e., Obamacare]; Medicare/Tricare; unknown or not reported; uninsured/no insurance), last visit with reproductive health care provider (in years), reproductive health status (premenopausal; menopausal; postmenopausal), oral contraceptive use (yes; no), and substance use (alcohol - number of drinks per week; tobacco/cigarettes - number of packs per day; illicit drugs, i.e., marijuana, cocaine, heroin, prescription medications (not taken as directed by a health care provider)- times per week). Participants were also questioned about their general health status, by asking them to list current diagnosed medical conditions or diseases, along with family history of specific mental health conditions (i.e., depression, emotional, substance-related, addiction disorders,). Lastly, participants were asked to list their religious preference (i.e., Buddhism, Christianity, Islam, or Judaism). 


\section{The Sexual Quality of Life-Female (SQOL-F) Questionnaire}

Sexual health, in this study, is defined as the way in which individuals demonstrate their knowledge, understanding, and tolerance of sexuality through behavior, emotion, and personal ideals. Sexual health was operationalized using total scores on the Sexual Quality of Life - Female (SQOL-F) questionnaire (Symonds et al., 2005). Quality of life (QOL), a construct developed by Walter Spitzer (1987), is one that is multidimensional and incorporates emotional, social, psychological, and physical domains as an appraisal of overall health and/or general well-being (Symonds et al., 2005). The SQOL-F was developed as a questionnaire to determine the influence of sexual dysfunction on a woman's life satisfaction or QOL (Maasoumi et al., 2013; Symonds et al., 2005). Sexual dysfunction, a concept often used in literature to identify sexual ill-health, has been used in the SQOL-F to recognize aspects of function that impact specific dimensions of QOL (Symonds et al., 2005). Originating from widely accepted approaches to sexual health, assessing sexual dysfunction maintains the perspective that sexuality and sexual relationships are affected by several factors including: psychological, cognitive, cultural, socio-economic, religious and political (World Health Organization, 2010).

The SQOL-F questionnaire briefly measures sexual quality of life by assessing, emotional and relationship issues along with self-esteem as three areas that may be impacted by sexual dysfunction (Symonds et al., 2005). The self-report instrument contains 18 items that are scored on a six-point Likert-like scale. Instrument responses are arranged in either ascending or descending order with options that include: 1) "completely agree", 2)"moderately agree", 3) slightly agree", 4) "slightly disagree", 5) "moderately disagree", and 6) "completely disagree" (Maasoumi et al., 2013; Symonds et al., 2005). The items, which specifically evaluate sexual confidence, emotional wellbeing, and relationship concerns are totaled, then normalized to a 1 to 100 point scale, not measuring separate domains (Pfizer Incorporation, 2014; Symonds et al., 2005). Higher total scores on the SQOL-F are indicative of a better sexual QOL (Symonds et al., 2005).

Both qualitative and quantitative studies have been carried out to determine the instrument's various psychometric properties. The first, a qualitative study was conducted to establish items to be included on the initial version of the instrument (Symonds et al., 2005). Semi-structured interviews were conducted using a sample of 82 women ranging in age from 19 to 65 years and residing in 7 different countries including the United States and United Kingdom (Symonds et al., 2005). Responses from the 60 to 90 minute interviews were analyzed to identify recurring themes, which were compiled and assembled to compose a 19-item measure quantifying the impact of sexual dysfunction on women's QOL. A literature review was also completed to verify clinical and conceptual relevance to sexual function and QOL (Symonds et al., 2005).

Three quantitative studies were also conducted on a total sample of 730 women to examine the questionnaire's validity and reliability (Symonds et al., 2005). Several psychometric tests including factor analysis, convergent and known-groups validity, internal consistency and test-retest reliability were completed to determine suitability for 
use in various populations (Pakpour, Zeidi, Saffari, \& Burri, 2013; Symonds et al., 2005). Factor analysis was completed to identify domains and validate each item that remained on the questionnaire. Empirical evidence was used to establish criteria to include those items with Eigen values greater than 1, factor loadings greater than 0.4, and Pearson's correlation (r) greater than 0.3 (Symonds et al., 2005). Based upon these criteria, one item, Question 18 -"When I think about my sexual life, I worry that my partner is looking for someone else" was eliminated (Symonds et al., 2005). Tests for internal consistency followed the factor analysis with an estimated Cronbach's Alpha of 0.95 (Symonds et al., 2005).

Convergent validity was verified by assessing correlations between SQOL-F total scores, specific items on the Life Satisfaction Checklist ("Satisfaction with partner relationship"; "Satisfaction with sex life") (Fugl-Meyer, Lodnert, Branholm, \& FuglMeyer, 1997) and the Sexual Function Questionnaire (SFQ) (Quirk et al., 2002). Correlations among SQOL-F and the previously mentioned measures were found to be significant for three groups of women, including a sample with spinal cord injury, one with female sexual dysfunction, and a group with normal sexual function (Symonds et al., 2005). Significant relationships were demonstrated between sexual QOL and sexual function with correlations noted between 0.5 and 0.7 for women in all groups (Symonds et al., 2005). Known-groups validity was also confirmed by comparing SQOL-F total scores between all three groups of women (Symonds et al., 2005). Results indicated that women with normal sexual function had an improved sexual QOL (SQOL-F score 90.1) when compared to women with either spinal cord injury (SQOL-F score - 63.3) or female sexual dysfunction (SQOL-F score - 59) (Symonds et al., 2005).

The final study was conducted to examine the SQOL-F's test-retest reliability. This study, with an identified power of $80 \%$ was used to recognize differences between SQOL-F scores obtained at two different time points (Symonds et al., 2005). With the time between test and retest averaging slightly over 13 days, the mean difference between SQOL-F scores was 0.52 with an intraclass correlation coefficient of $0.85(p<0.0001)$ (Symonds et al., 2005). Both results indicate that obtained SQOL-F scores were highly and significantly correlated $(r=0.6)$ (Rosner, 2011; Symonds et al., 2005). These and previously mentioned tests render substantial evidence to support use of the SQOL-F as an effective measure of sexual health in this study.

\section{The Sexual Activities and Attitudes Questionnaire (SAAQ)}

Sexual risk behavior, an additional outcome variable examined in this study was quantified using the Sexual Activities and Attitudes Questionnaire (SAAQ) (Noll et al., 2003). For the purposes of this study, sexual risk behavior included participation in any activity that may involve the following: having unprotected vaginal or anal sex, participating in unprotected oral sex, substance use, and/or sharing drug use equipment, trading sexual favors for money or other means of support, engaging in concurrent sexual partnerships, and being involved in actual or potential partner violence (Centers for Disease Control and Prevention, 2014a; Whyte, 2005). The SAAQ is a self-report 
measure containing 44-items that assess both sexual activities and sexual attitudes (Noll et al., 2003). Due to the instrument's sensitive nature, developers assessed sexual attitudes along with behaviors in an effort to improve the measure's ability to obtain accurate information (Noll et al., 2003). Sexual attitudes were examined by asking participants to respond to instrument items by reflecting upon only those sexual activities that were engaged in willingly, rather than those that were involuntary or forced (Noll \& Shenk, 2013; Noll et al., 2003). Initial instrument items are scored on a five-point Likert scale arranged using divergent questioning, which instructs participants to respond to items based upon previous responses.

The SAAQ includes seven variables to measure sexual activity. Activities assessed include the following: 1) age at first voluntary sexual intercourse, 2) birth control efficacy (current), 3) sexual intercourse partners (number within the last year), 4) HIV-risk behaviors (risks include having sexual intercourse: unprotected, while under the influence of drugs or alcohol, with IV drug users, during a "one-night stand), 5) STIs (including gonorrhea, chlamydia, syphilis, genital herpes, genital warts, hepatitis B and C), 6) pregnancies (history, birth control efficacy - previous), and 7) sexual behaviors of peers (Noll et al., 2003).

The SAAQ also includes items that measure sexual attitudes. Sexual attitude factors found to be reliable from factor analysis conducted to assess risky sexual behaviors included the following: 1) sexual preoccupation $(\propto=.91)$ - positive attitudes toward/frequency of participating in activities including: masturbation, use of pornographic media, and having sexual thoughts; 2$)$ sexual permissiveness $(\propto=.96)$ tolerance toward a regulating or normalizing set of needs/behaviors including: intimate feeling, touching, and voluntary sexual intercourse; 3 ) internal and external pressure to engage in $\operatorname{sex}(\propto=.70)$ - thoughts that sexual activity is expected, that one will be accepted, or will gain respect/love for self and from others if sexual activity is engaged; and 4) negative attitude toward sex $(\propto=.85)$ - beliefs that sexual activity is "dirty" or will result in losing respect for self and from others (Noll, 2003; Noll et al., 2003). Low factor correlations as compared to reliability as indicated from Cronbach's alpha reveal that factors are in fact independent, with constructs being slightly related (Noll et al., 2003; Rosner, 2011). Two additional factors, sexual aversion and sexual ambivalence may also be assessed using prediction equations as follows: 1) sexual aversion $=-1 \mathrm{x}$ (sexual permissiveness) + (negative attitude toward sex), and 2) sexual ambivalence $=$ (sexual preoccupation) + (sexual aversion) (Noll et al., 2003).

Predictive and discriminant validity were confirmed while assessing sexual attitudes and activities using responses on the SAAQ for 77 sexually abused women and a comparison group of 89 women (Bouchard, Godbout, \& Sabourin, 2009; Noll et al., 2003). Results revealed childhood sexual abuse and anxiety as significant predictors of sexual preoccupation $(\beta=.30, p<.05)(\beta=.23, p<.05)$ (Noll et al., 2003). Additionally, childhood sexual abuse was a significant predictor of pathological dissociation $(\beta=.36, p<.01)$ (Noll, 2003). A comparison of responses for women in both groups demonstrated differences for abused women with greater sexual preoccupation $(F=3.98, p=.04)$, younger age at first voluntary sexual intercourse $(F=$ 
$15.18, p=.0002)$, and lower birth control efficacy $(F=11.66, p=.0008)$ (Noll et al., 2003).

Instrument developers found that cohabitation or marital status could potentially affect responses to instrument items, specifically those related to number of sexual partners or frequency of sexual activity (Noll et al., 2003). Another issue having the potential to affect participant responses included the instrument construct, sexual permissiveness. This factor, was considered "age-sensitive" by developers, which could also affect responses related to this factor (Noll et al., 2003). For both of these reasons, cohabitation/marital status and age were statistically controlled by using multivariate analysis of variance (MANOVA) in conducted analyses (Noll et al., 2003). After controlling for these variables, the SAAQ was found to be a consistent and valid instrument to assess sexual activities and attitudes in participants of varying ages (Bouchard et al., 2009; Noll et al., 2003).

\section{The Brief Multidimensional Measure of Religiousness/Spirituality (BMMRS)}

In this study, both spirituality and religiosity were recognized as emotional factors having some influence on the sexual health and behavior of AAW. Spirituality, in this study is recognized as an emotional factor and defined as the state in which individuals address their own ethical or moral concerns. While closely related to spirituality, religiosity was defined as the manner in which religion or religious constructs affect the way an individual achieves balance between their ethical and sexual selves (Robinson et al., 2002). Both concepts were operationalized in this study using the Brief Multidimensional Measure of Religiousness/Spirituality (BMMRS).

The BMMRS is a 38-item, self-report measure designed to distinguish between the concepts of spirituality and religiosity while measuring their connection to health (Fetzer Institute \& National Institute on Aging Working Group, 1999; Johnstone, McCormack, Yoon, \& Smith, 2012; Johnstone, Yoon, Franklin, Schopp, \& Hinkebein, 2009). The instrument was developed under the assumption that religiousness and spirituality are linked to health through behavioral, social, psychological, and physiological factors (Fetzer Institute \& National Institute on Aging Working Group, 1999). The instrument includes 12 domains each selected by developers based upon their theoretical or practical influence on health outcomes (Fetzer Institute \& National Institute on Aging Working Group, 1999; Johnstone et al., 2009; J. Williams et al., 2012). Instrument items vary from being scored on a four- to eight-point Likert-type scale, and also include dichotomous (yes/no) and open ended, short answer questions (Harris et al., 2008).

Domains included on the BMMRS to assess several facets of religiosity and spirituality include the following: 1) daily spiritual experiences; 2) meaning; 3) values; 4) beliefs; 5) forgiveness; 6) private religious practices; 7) religious/spiritual coping; 8) religious support; 9) religious/spiritual history; 10) commitment; 11) organizational religiousness; and 12) religious preferences (Fetzer Institute \& National Institute on 
Aging Working Group, 1999; Johnstone et al., 2012; Johnstone et al., 2009). A detailed description of BMMRS domains is listed in Table 3-1. Developers acknowledge that this brief multi-dimensional measure may be used in its entirety or its subscales used as a measure of selected individual domains (Fetzer Institute \& National Institute on Aging Working Group, 1999; Shim et al., 2013).

Preliminary reliability and validity of the BMMRS was established from embedding the instrument into a National Data Program for the Social Sciences random survey (Fetzer Institute \& National Institute on Aging Working Group, 1999; Shim et al., 2013). Findings from the survey demonstrate the BMMRS's various psychometric properties and support its multidimensional approach (Fetzer Institute \& National Institute on Aging Working Group, 1999). Additional factor analytic studies have been carried out to confirm the instrument's ability to distinguish between its spiritual and religious constructs (Johnstone et al., 2012). Of the number of factors found to differentiate spirituality from religion, those most frequently identified include the following: spirituality, spiritual distress, religious practices/religiosity, congregational support, and meaning (Johnstone et al., 2009; Piedmont, Mapa, \& Williams, 2007). Internal reliability for each of the instrument's domains has also been confirmed in previous studies with results ranging from an estimated Cronbach's alpha of .71 to .91 (Fetzer Institute \& National Institute on Aging Working Group, 1999; Johnstone et al., 2009). Convergent validity of the instrument's spiritual domains was found to be significantly correlated with the Temperament and Character Inventory (TCI), a tool developed to measure temperament and character (Johnstone et al., 2012; Van Dam \& Earleywine, 2011). Likewise, divergent validity of religious domains (religious practices) were not found to be significantly correlated with specific scales on the TCI, indicating that the BMMRS's religious and spiritual constructs are in fact distinct (Johnstone et al., 2012).

The completed analyses confirm reliability and validity for each of the instrument's domains and support its use in both young and older adults (Fetzer Institute \& National Institute on Aging Working Group, 1999; Johnstone et al., 2012; Johnstone et al., 2009; Radloff, 1977). Recent psychometric testing has also been conducted on a Portuguese version of the BMMRS and additionally with a sample of 180 Irish undergraduate students (Shim et al., 2013; J. Williams et al., 2012). Previous and recent analyses suggest that the BMMRS is a useful tool to assess various aspects of religiosity and spirituality in both clinical and non-clinical samples (Shim et al., 2013; J. Williams et al., 2012).

\section{The Center for Epidemiologic Studies Depression Scale Revised}

Depressive symptomology is also being examined in this study as an emotional factor with potential influence on sexual health and risk behavior. This concept has been defined as the presence of symptoms that includes changes in: sleep, appetite, ability to concentrate, level of energy, interest, self-esteem, or feelings of guilt. Depression 
Table 3-1. Description of Brief Multidimensional Measure of Religiousness / Spirituality Domains.

\begin{tabular}{|c|c|}
\hline BMMRS Domains & Domain Description \\
\hline Daily spiritual experiences & $\begin{array}{l}\text { An individual's perception of/interaction with a } \\
\text { transcendent in daily life. }\end{array}$ \\
\hline Meaning & $\begin{array}{l}\text { An individual's search for and their success and } \\
\text { failure in that search for understanding of life. }\end{array}$ \\
\hline Values & $\begin{array}{l}\text { The degree to which an individual's behavior } \\
\text { reflects their faith or religion. }\end{array}$ \\
\hline Beliefs & $\begin{array}{l}\text { An individual's acceptance of religious/spiritual } \\
\text { convictions. }\end{array}$ \\
\hline Forgiveness & $\begin{array}{l}\text { An individual's perception of being able to forgive } \\
\text { oneself and others, being forgiven by others and by } \\
\text { God, and confession. }\end{array}$ \\
\hline Private religious practices & $\begin{array}{l}\text { The degree of an individual's religious involvement } \\
\text { through habits or participation in rituals. }\end{array}$ \\
\hline Religious/spiritual coping & $\begin{array}{l}\text { Positive and negative coping mechanisms based on } \\
\text { religious/spiritual ways of managing life stressors. }\end{array}$ \\
\hline Religious support & $\begin{array}{l}\text { An individual's personal relationships established } \\
\text { based upon shared religious/spiritual beliefs. }\end{array}$ \\
\hline Religious/spiritual history & $\begin{array}{l}\text { An individual's past experience with participation } \\
\text { in religious/spiritual activity over the course of } \\
\text { one's life. }\end{array}$ \\
\hline Commitment & $\begin{array}{l}\text { The significance and dedication to one's } \\
\text { religious/spiritual beliefs. }\end{array}$ \\
\hline Organizational religiousness & $\begin{array}{l}\text { An individual's involvement with formal religious } \\
\text { establishments or associations. }\end{array}$ \\
\hline Religious preferences & $\begin{array}{l}\text { An individual's inclination toward their religious } \\
\text { denomination. }\end{array}$ \\
\hline
\end{tabular}

Note. BMMRS...... Brief Multidimensional Measure of Religiousness/Spirituality 
symptoms will be quantified in this study using scores on the Center for Epidemiologic Studies Depression Scale Revised (CESD-R) (Eaton et al., 2004).

The CESD-R is 20-item, self-report measure designed to identify symptoms of depression and depressive disorder (Center for Innovative Public Health Research, 2014; Shim et al., 2013; Van Dam \& Earleywine, 2011). The instrument in its original form, the Center for Epidemiologic Studies Depression Scale (CESD), is a heavily used measure of depressive symptomology that over time has become obsolete (Eaton et al., 2004; Radloff, 1977; Van Dam \& Earleywine, 2011). Despite exhibiting good psychometric properties for use in the general population, a significant limitation of the CESD includes being based on the American Psychiatric Association Diagnostic and Statistical Manual, second edition (DSM-II) (Eaton et al., 2004; Radloff, 1977).

Revised to accommodate modern diagnostic criteria, the CESD-R uses nine groups as defined by the American Psychiatric Association Diagnostic and Statistical Manual, fourth edition (DSM-IV) to distinguish symptoms of depression or depressive disorder (Center for Innovative Public Health Research, 2014; Eaton et al., 2004; Van Dam \& Earleywine, 2011). The nine domains used to assess depressive symptoms include the following: 1) sadness, 2) loss of interest, 3) appetite, 4) sleep, 5) thinking/concentration, 6) guilt, 7) tired, 8) movement, and 9) suicidal ideation (Center for Innovative Public Health Research, 2014; Eaton et al., 2004; Van Dam \& Earleywine, 2011). Instrument items assessing the frequency of symptoms in these areas are scored on a 5-point Likert scale. Item responses reflecting upon the occurrence of symptoms experienced within "the past week", include the following: 1) "less than one day/not at all”, 2) "1-2 days", 3) "3-4 days", 4) "5-7 days", and 5) "nearly every day for 2 weeks (Eaton et al., 2004). Scores for each response are totaled to determine categories of possible depressive symptoms. Categories for depressive symptoms are based largely on the frequency of specific symptom groups and include the following: 1) "no clinical significance", 2) "subthreshold depression symptoms", 3) "possible major depressive episode", 4)"probable major depressive episode", and 5) "meets criteria for major depressive episode" (Center for Innovative Public Health Research, 2014; Eaton et al., 2004).

The revised version of the CESD-R demonstrates sound reliability and validity through statistical measures that include: exploratory and confirmatory factor analyses, convergent and divergent validity, and established classification schemes or algorithms (Van Dam \& Earleywine, 2011). High internal consistency (Cronbach's $\alpha=$ $0.923 ; 0.928)$ was demonstrated when the instrument was tested on two separate samples $(n=10,304 ; n=245)$ of men and women with an average age of 30 and 19 years, respectively (Van Dam \& Earleywine, 2011). Exploratory factor analysis was conducted using two-factor solution based on Maximum Likelihood Estimation. This analysis revealed psychometric consistency with one factor related to functional impairment and a second factor related to negative mood (Van Dam \& Earleywine, 2011). Confirmatory factor analysis indicated a good model fit for both samples with inter-factor correlations of 0.941 and 0.975, respectively (Van Dam \& Earleywine, 2011). 
Convergent and divergent validity of the CESD-R was confirmed by comparing the instrument with other questionnaires used to identify conditions that commonly occur with depression to include: anxiety, positive affect, and negative affect (Van Dam \& Earleywine, 2011). Positive correlations between the CESD-R and the State-Trait Inventory for Cognitive and Somatic Anxiety (STICSA), an instrument used to distinguish cognitive and somatic factors associated with anxiety for both samples $(r=$ $0.737, p<.001 ; r=0.653, p<.001$, respectively), in addition to the Positive and Negative Affect Schedule - Negative Affect (PANAS-NA), an instrument used to measure negative affect $(r=0.576, p<.001)$, reveal the instrument's convergent validity (Ree, French, MacLeod, \& Locke, 2008; Van Dam \& Earleywine, 2011). Conversely, divergent validity was confirmed by comparing the CESD-R with the Positive and Negative Affect Schedule - Positive Affect (PANAS-PA), with a negative correlation ( $r$ $=-0.263, p<.001)$ (Van Dam \& Earleywine, 2011). Lastly, the algorithm used to classify depressive symptom categories demonstrates improved specificity in the identification of depressive symptoms from that of the CESD (Van Dam \& Earleywine, 2011).

The above stated findings support use of the CESD-R as a precise measure of depressive symptoms within the general population (Van Dam \& Earleywine, 2011). While both samples used for psychometric testing include small subsets of self-identified African Americans $(1.3 \% ; 8.2 \%)$, the CESD-R has been used with AAW in varied settings including primary care and community-based (Shim et al., 2013; Van Dam \& Earleywine, 2011; J. Williams et al., 2012). For the purposes of this study, total scores on the CESD-R will be used as a measure of depressive symptomology.

\section{The Sexual Relationship Power Scale}

In this study, gender inequality was examined as a relational factor acknowledged to moderate difficulty asserting, negotiating, or making decisions regarding sexual health and risk behaviors (Robinson et al., 2002). Gender inequality, an important concept related to women's sexual health, has been defined as differences in power that cause inequities between men and women (McMahon, Volpe, Klostermann, Trabold, \& Xue, 2014; Pulerwitz et al., 2000). Gender inequality was operationalized in this study using scores on the Sexual Relationship Power Scale (SRPS) (Pulerwitz et al., 2000).

The SRPS, developed by Pulerwitz, Gortmaker, and DeJong, is a 23-item measure of issues occurring within relationships (relationship dynamics) that contain two domains or subscales of relationship power including: 1) relationship control, and 2) decisionmaking dominance (Matsuda, McGrath, \& Jallo, 2012; Pulerwitz et al., 2000). The relationship control subscale (15 items) focuses on questions related to participants' perception of their partner's control, while the decision making dominance subscale (8 items) asks questions about the partner having greater influence on decisions made in the participant's every life (Matsuda et al., 2012; Pulerwitz et al., 2000). Instrument items included on the relationship control subscale are scored on a 4-point Likert-like scale with responses including the following: 1) "strongly disagree", 2) "disagree", 3) "agree", and 4) "strongly agree" (Matsuda et al., 2012; Pulerwitz et al., 2000). The decisionmaking dominance subscale is scored on a 3-point Likert-like scale with responses as 
follows: 1) "you”, 2) "both of you equally", and 3) "your partner" (Pulerwitz et al., 2000). Scores for each response are totaled to determine categories that represent low, medium, and high levels of sexual relationship power (Pulerwitz et al., 2000).

Psychometric testing including content and face validity were carried out to establish factors deemed relevant by community members, HIV/AIDS educators, and students (Pulerwitz et al., 2000). Literature reviews, focus groups, and factor analysis generated support for items included on the final instrument, including its two distinct domains (Pulerwitz et al., 2000). Empirical evidence used for item inclusion was a factor loading of less than 0.30 (Pulerwitz et al., 2000).

Both domains included on the SRPS, demonstrate good reliability and validity with conducted analyses based on factor analysis and construct validity (Matsuda et al., 2012; McMahon et al., 2014; Pulerwitz et al., 2000). Internal consistency was confirmed for SRPS subscales with an estimated Cronbach's $\alpha$ used as a measure of analysis (relationship control: $\alpha=0.86$; decision-making dominance: $\alpha=0.63$ ) (Matsuda et al., 2012; Pulerwitz et al., 2000). Instrument developers acknowledge flexibility of the instrument to be administered to measure separate domains/subscales or together to generate an overall score (Matsuda et al., 2012; Pulerwitz et al., 2000). Good internal consistency for the overall scale has also been demonstrated (estimated Cronbach's $\alpha=$ .84 (Pulerwitz et al., 2000). Predictive and construct validity was also demonstrated by testing the relationship between the SRPS and specific factors assumed to be related to relationship power (Pulerwitz et al., 2000).

Critical analysis conducted on the SRPS reveal the instrument to be an acceptable measure of relationship power for both men and women (Matsuda et al., 2012; McMahon et al., 2014). In addition to being developed in part for AAW, psychometric testing has been carried out on an adult sample of AAW demonstrating reliability of the overall instrument (Cronbach's $\alpha=0.89$ ) (Matsuda et al., 2012; McMahon et al., 2014). The fact that the instrument reflects current sexual behaviors and attitudes has established the SRPS as a dependable measure of power in sexual relationships (Matsuda et al., 2012; McMahon et al., 2014). Both domains, relationship control and decision-making dominance will be combined to determine a total score. For the purposes of this study, total scores on the SRPS will be used as a measure of gender inequality.

Table 3-2 displays the reliability coefficients and time required for participants to complete each of the instruments used to quantify emotional, relational, and outcome variables being examined in this study.

\section{Procedure}

The University of Tennessee Health Science Center's Institutional Review Board (UTHSC IRB) granted permission to conduct the proposed research study. All study procedures including data collection and respondent validation were carried out between July and December 2015. The principal investigator initiated study procedures with the 
Table 3-2. Instrument Reliability and Completion Times.

\begin{tabular}{|c|c|c|c|}
\hline Instrument & Number of Items & $\begin{array}{l}\text { Reliability } \\
\text { Coefficients }\end{array}$ & $\begin{array}{l}\text { Minutes to } \\
\text { Complete }\end{array}$ \\
\hline $\begin{array}{l}\text { Sexual Quality of Life } \\
\text { (SQOL-F) Questionnaire } \\
\text { (Symonds et al., 2005) }\end{array}$ & 18 & .85 & $7-10$ \\
\hline $\begin{array}{l}\text { Sexual Activities and } \\
\text { Attitudes Questionnaire } \\
\text { (SAAQ) } \\
\text { (Noll et al., 2003) }\end{array}$ & 44 & .86 & $20-25$ \\
\hline $\begin{array}{l}\text { Brief Multidimensional } \\
\text { Measure of } \\
\text { Religiousness/Spirituality } \\
\text { (BMMRS) } \\
\text { (Fetzer Institute \& } \\
\text { National Institute on } \\
\text { Aging Working Group, } \\
\text { 1999) }\end{array}$ & 38 & $.71-.91$ & $15-17$ \\
\hline $\begin{array}{l}\text { Center for Epidemiologic } \\
\text { Studies Depression Scale } \\
\text { Revised (CESD-R) } \\
\text { (Eaton et al., 2004) }\end{array}$ & 20 & $.923-.928$ & $7-10$ \\
\hline $\begin{array}{l}\text { Sexual Relationship } \\
\text { Power Scale (SRPS) } \\
\text { (Pulerwitz et al., 2000) }\end{array}$ & 23 & .89 & $10-15$ \\
\hline Total time & & & $59-75$ \\
\hline
\end{tabular}


screening of potential study subjects recruited through the use of flyers, posters, email listservs, or participant referrals to confirm eligibility and willingness to participate. Screening interviews included explanations of the study and study procedures, inclusion and exclusion criteria, and confidentiality and participant rights. After confirming decisions regarding participation, informed consent was obtained during face-to-face, private meetings with study subjects. Participants were then asked to complete instruments in their entirety and offered assistance or clarification with specific instrument items, if needed. Potential participants who were unavailable for face-to-face meetings were consented over the telephone and had informed consent forms mailed with addressed envelopes and provided postage for forms to be returned to the principal investigator. Once instruments were completed, study participants received a \$20.00 gift card to their choice of one of two retail stores. After receiving compensation for participation, study subjects were asked to participate in focus group interviews occurring at a later date.

The study investigator contacted selected individuals who previously expressed potential interest in completing the study's QUAL component. Prior to focus group interviews, study subjects confirmed informed consent and received an additional explanation of QUAL study procedures. During 60 to 90 minute long focus group interviews, participants were asked structured, open-ended questions within groups of 9 to 10 individuals. Participants were given the opportunity to respond freely to topics concerning their sexual health and factors influencing their sexual behavior. Study investigators conducted interviews using a pre-written script and interview guide. Digital recording devices were used to record focus group interview sessions, which were later transcribed and entered into NVivo 10 software (QSR International [Americas], Burlington, MA) for analysis. Once QUAL study procedures were completed, participants received a $\$ 20.00$ gift card to their choice of one of two retail stores.

To ensure validity of the study's qualitative study findings, respondent validation procedures were carried out for selected QUAL study participants. Additional informed consent was obtained from solicited participants to undergo procedures to validate the completed analyses of data collected during focus group interviews. For respondent validation, participants completed individual dissemination sessions with the study investigator. During dissemination sessions, participants were provided with a 1-page written summary report of investigators' interpretation of QUAL study findings. After reviewing the summary report, participants were given the opportunity to approve or disapprove the accuracy of investigators' interpretation of women's thoughts and feelings. After completing validation procedures, participants received a $\$ 20.00$ gift card to their choice of one of two retail stores.

Data collected during study visits were labeled with study identification numbers assigned to each participant. Identifying information included on data obtained from participants was kept confidential and securely stored by the principal investigator. 


\section{Data Analysis}

Quantitative data collected from study participants were entered into Microsoft ${ }^{\circledR}$ Excel ${ }^{\circledR}$ and imported into Statistical Analysis Software (SAS) version 9.4 (SAS Institute Inc., Cary, NC) for analyses. Initially, descriptive statistics (measures of central tendency, counts, frequency, mean and standard deviation distributions) were estimated to provide descriptive statistics, which were used to describe the study's sample. The relationships among specified emotional and relational factors and both outcome variables — sexual health and sexual risk behavior-were estimated using Pearson's product-moment correlation coefficients (r) and subsequently multiple linear regression models were fitted.

Specific aim one is to determine the associations between spirituality/religiosity and (a) sexual health in AAW and (b) sexual risk behavior in AAW. Scores from the SQOL-F and subscales from the BMMRS and SAAQ were analyzed using descriptive statistics. For all variables assumption regarding normality was met. Estimated measures of central tendency were determined (means and medians). Correlational analyses using Pearson's product moment correlation coefficients estimated associations between spirituality/religiosity and each of the outcome variables sexual health and sexual risk behavior. Subsequently, associations among age, relationship status, and educational level were used to estimate by Pearson's product moment correlation coefficients. Because of the exploratory nature of this study, an alpha of 0.20 was chosen for statistical significance.

Specific aim two is to determine the associations between depressive symptomology and (a) sexual health in AAW and (b) sexual risk behavior in AAW. Scores from the CESD-R and SQOL-F and subscales from SAAQ were analyzed using descriptive statistics. As previously mentioned, estimated measures of central tendency were determined (means and medians). Correlational analyses using Pearson's product moment correlation coefficients estimated associations between depressive symptomology and each of the outcome variables, sexual health and sexual risk behavior.

Specific aim three is to determine the associations between gender inequality and (a) sexual health in AAW and (b) sexual risk behavior in AAW. Scores from the SRPS and SQOL-F and subscales from SAAQ were analyzed using descriptive statistics. Since data were determined to be generated from a normal underlying distribution, correlational analyses using Pearson's product moment correlation coefficients were calculated to determine the presence of relationships between gender inequality and each of the outcome variables, sexual health and sexual risk behavior.

Specific aim four is to determine the extent to which spirituality/religiosity, depressive symptomology, and gender inequality influence (a) sexual health in AAW and (b) sexual risk behavior in AAW. Simple and multiple regression analyses were used to analyze scores from the BMMRS, CESD-R, SRPS, SQOL-F, and SAAQ. Based on previously conducted analyses to estimate the linear relationships between various independent variables and sexual health and sexual risk behavior, selected independent 
variables were entered into multiple linear regression analyses as potential predictors. Both emotional and relational factors were additionally analyzed using simultaneous multiple regression to determine their influence on sexual health and participation in sexual risk behavior. Tests for multicollinearity were also conducted to determine the adequacy of variables included in multiple regression models.

Qualitative data gathered during focus group interviews were used to address questions posed within specific aim five. QUAL data were transcribed and analyzed using an inductive approach where codes were developed into categories, which were used to generate over-arching themes. Analysis involved thematic content analysis.

Specific aim five is to examine a variety of AAW's perceptions of sexual health. Focus group interviews were transcribed and checked for accuracy by recording and listening to taped interviews. Data were and analyzed using thematic analysis to identify themes emerging from obtained data. Interview transcripts were entered into NVivo 10 software (QSR International [Americas], Burlington, MA). QUAL data were reduced by coding conducted by the study investigator. Following the process of coding, data were clustered into categories, and categories were merged into themes. QUAL themes were validated with study participants, as well as by members of the research team. Final themes were generated from the completion of multiple comparisons of codes were used to describe participants' perception of sexual health and influences on their sexual behavior.

\section{Consideration of Human Subjects}

Consideration was given to ensure the safety and protection of participants involved in all aspects of this study. Procedures were carried out to address principles of justice, respect for human dignity, and beneficence for women who were recruited and agreed to study participation. Study procedures were reviewed and approved by the Institutional Review Board at the University of Tennessee Health Science Center (Appendix A).

Upholding justice for study participants involved the provision of fair treatment and preservation of privacy. The beliefs and decisions of participants were respected, while each was treated in a non-judgmental manner. Due to the sensitive nature of topics being examined in this study, efforts were be made to design questions in such a way that the level of intrusion did not surpass that necessary to sufficiently address the study's aims. A certificate of confidentiality was obtained through the National Institutes of Child Health and Human Development. Obtaining this certificate is suitable for any research involving the collection of sensitive, identifiable data, and can serve to protect participants from forced disclosure should study records be subject to legal subpoena (Polit \& Beck, 2012).

Additional measures to ensure participants' confidentiality were implemented in the security of data obtained from study participation. Collected data were labeled with 
study identification numbers, which were assigned to each participant. Materials linking identification numbers to participants were securely stored and password protected. Identifiable data were only accessible to the study's principal investigator.

In this study, respecting the human dignity of study participants involved protecting their rights to self-determination and full disclosure. Informed consent was used to address these components. Consent forms were developed to provide a detailed description of study procedures, explain risks and benefits associated with participation, and give a statement of agreement to the terms of participation. The consent forms were approved by the UTHSC IRB to carry out procedures used to collect both quantitative and qualitative data to include completing questionnaires and focus group interviews. Informed consent was given prior to participants initiating any aspect of the study (Appendix B). Additional explanation was given to each study participant regarding her ability to make decisions to participate and/or withdraw from the study at any time. Participants were also informed of responsibilities of the principal investigator.

Lastly, procedures carried out to address beneficence involved protecting participants' right to freedom from harm and protection from exploitation. An evaluation of risks and implementation of measures to protect study participants from identified risks was used to address these components. Both psychological and legal risks may be ensued from participation in this study. Minimal psychological risk may be posed to participants due to stigma associated with the study's subject matter. Study participants were asked questions of a highly personal nature regarding fairly sensitive topics (sexual behavior, STIs, intimate partner violence) either through interviewing or completion of questionnaires. Legal risks for study subjects could have been encountered from the completion of questionnaires that involved disclosure of illegal activity. The admission of such information could pose legal risk if researchers/investigators were required by law (search warrant or subpoena) to disclose information.

Actions to minimize psychological and legal risk included informing subjects' of their right to refuse participation in any aspect of this research study. Study subjects were informed of their ability to omit answering questions or completing questionnaires that might cause emotional distress. In the case that study subjects experienced emotional distress related to completion of study instruments, referrals would have been made to a local metal health care provider to obtain mental health services. Subjects were informed of their right during the informed consent process and prior to initiating any interview or completing questionnaires. During the informed consent process, subjects were made aware of public law as it relates to disclosure of information obtained from participation in a research study. Every effort was made to obtain minimal amounts of identifying information and any information regarding potentially illegal activity, as necessary to the conduct of this research study. 


\section{CHAPTER 4. RESULTS}

\section{Introduction}

When looking at the health status of our nation from a public health perspective, an examination of sexual and reproductive health reveals that there are multiple conditions posing a burden on the health of African American women (AAW). Conditions identified as potential threats to AAW's health include: sexually transmitted infections (STIs), viral hepatitis, teen/unintended pregnancy, and sexual violence (Centers for Disease Control and Prevention, 2014a). Current health promotion efforts have been unsuccessful in curtailing disparities in sexual and reproductive health for AAW.

At some point in their lifetimes, an estimated 1 in 32 African American women will be diagnosed with HIV infection. Despite recent decreases in infection rates within this group, in 2011 AAW accounted for $64 \%$ of new HIV infections in women (Centers for Disease Control and Prevention, 2014c). Similarly, disparities can also be seen in recent teen birth rates, as well as, gonorrhea and chlamydia infection rates (Centers for Disease Control and Prevention, 2014f; Martin et al., 2013). Teen birth rates for African Americans in 2011 were twice the rate of non-Hispanic white teens (Ventura et al., 2014; Ventura et al., 2011). STI surveillance data for the same year reveal gonorrhea infection rates among AAW over 15 times that of white women and chlamydia rates that were over six times higher than the rate among non-Hispanic white women (Centers for Disease Control and Prevention, 2014a).

High risk behaviors related to sexual activity, often referred to as sexual risk behaviors, are those that increase an individual's potential of acquiring STIs and experiencing unintended pregnancy (Centers for Disease Control and Prevention, 2014e; Lutfi, Trepka, Fennie, Ibanez, \& Gladwin, 2015). Patterns of sexual behavior distinct to racial or ethnic groups are hypothesized to differentiate risks of experiencing negative health outcomes (Adimora et al., 2013; D’Souza, Cullen, Bowie, Thorpe, \& Fakhry, 2014; Pfileger et al., 2013). For instance, groups identified as having increased risk of becoming infected with HIV are those recognized as participating in specific behaviors including the following: having multiple, high risk, male partners (males with unknown STI status, injection drug users, or males who have sex with other males); having concurrent partnerships; infrequently using condoms or other methods of protection; and engaging in a variety of sexual activities (Centers for Disease Control and Prevention, 2014b, 2014d).

Studies have shown that sexual risk behaviors unique to AAW include: inconsistent condom use or unprotected sex, early age of sexual initiation (before 15 years of age), range of sexual activity (anal, oral, or vaginal sex), and having concurrent sexual partners (McLellan-Lemal et al., 2012; Neblett et al., 2011). Factors contributing to the disparity in STIs and unintended pregnancy affecting AAW extend beyond participation in specific behaviors to include social and/or cultural components that 
characterize the life experiences of these women (Hunter \& Tilley, 2015; Sharpe et al., 2012). Exploring the composition of social networks, issues related to gender, and the promotion of sexual health may provide insight into specific factors continuing to compromise the health status of AAW (Adimora et al., 2013; Hall \& Pichon, 2014; Mincey \& Norris, 2014; Neblett et al., 2011; Pfileger et al., 2013).

Sexual health is a holistic approach that places emphasis on achieving a state of well-being and acknowledges the influence of sexual, social, emotional, and physical factors (Robinson et al., 2002; World Health Organization, 2002). Taking the broad approach toward sexual health, rather than the more traditional reproductive health approaches often used to develop existing health promotion strategies may be the key to addressing factors unique to AAW. For example, the mode of transmission attributable to most new HIV infections for AAW (87\%) is heterosexual sexual contact, a mode distinct from other groups heavily affected by HIV (Centers for Disease Control and Prevention, 2014c). Failure to recognize factors specific to AAW may allow disparate rates of infection and other negative health outcomes to persist for this group posing a threat to their immediate and long-term health. Having an increased risk for STIs may result in reproductive complications including pelvic inflammatory disease and infertility.

The purpose of this study is to better understand AAW's sexual health and factors contributing to the health behaviors placing this group at increased risk for acquiring STIs and unintended pregnancy. This mixed methods study used quantitative methods and analysis to describe the associations among selected emotional (i.e., religiosity/spirituality, depressive symptomology) and relational (i.e., gender inequality) factors and sexual health and sexual risk behavior in a population of AAW recruited from community settings. Additionally, qualitative data were collected to explore AAW's perception of sexual health. Both qualitative and quantitative data were analyzed, compared and contrasted to make inferences that may improve understanding of AAW's life experiences relative to sexual health and risk behavior.

\section{Methods}

In this mixed methods study, a convergent parallel design was employed to provide a comprehensive description of the multifaceted concept of sexual health and the complex factors involved in AAWs sexual behavior. The Sexual Health Model provides a conceptual foundation for this study. This foundation is one that is grounded in a holistic approach to sexual health that encompasses emotional, relational, and sexual factors thought to impact behavior and overall well-being (Robinson et al., 2002). Quantitative (QUAN) and qualitative (QUAL) data were collected concurrently and analyzed separately. QUAN and QUAL findings were merged, allowing for corroboration of findings through the comparison and contrast of results from both methods (Creswell \& Plano Clark, 2011). 


\section{Study Procedures}

Review and approval of study procedures were completed by the Institutional Review Board at the University of Tennessee Health Science Center. An urban community sample was recruited through the use of flyers, social media, e-mail listservs, and participant referrals. Screening interviews were conducted and included an explanation of the study and study procedures, confirmation of study eligibility, and explanations of confidentiality and participant rights. Criteria for study eligibility included the following: a) self-identified African American female, b) reported sexual activity within the past 12 months; c) Memphis metropolitan area resident; d) at least 18 years of age or older at the time of enrollment; and e) ability to read, speak, and understand English. After confirming decisions regarding study participation and eligibility, informed consent was obtained during face-to-face, private meetings with study subjects. All participants $(\mathrm{N}=50)$ completed the study's QUAN strand, which included an initial study visit involving the completion of questionnaires used to quantify variables hypothesized to increase risk for negative health outcomes associated with sexual health in AAW. Participants received their choice of one of two retail store gift cards for completion of the initial study visit.

Purposive sampling was used to obtain the study's QUAL sample $(\mathrm{N}=19)$. Focus group participants were conveniently selected from the sample of subjects who completed the study's QUAN component. Women who expressed interest in focus group participation during the enrollment interview were contacted by the study investigator to complete a focus group interview that was scheduled within six weeks of study enrollment and the initial study visit. Women selected for the focus group were recruited to reflect the age, relationship status, and reproductive health status of the sample. Focus group participants received an additional explanation of the study, study procedures, criteria for selection of focus group participants, confidentiality, and focus group interview guidelines. During the 60 to 90 minute focus group interviews, participants were asked open-ended questions within groups of 9 to 10 individuals. Semi-structured interviews were conducted using a pre-written script and interview guide. Interviews topics included participants' sexual health, and emotional, relational, and behavioral factors that may influence achieving this state of health (Figure 4-1). Participants were encouraged to respond freely to questions inquiring about their perception of sexual health. Focus group interviews were recorded using both a handheld computing device and digital voice recorder, transcribed, and imported into NVivo 10. Once focus group interviews were completed, participants received a retail store gift card.

Respondent validation procedures were carried out to ensure the validity of research findings obtained from the study's QUAL component. After study investigators analyzed and interpreted data from focus group interviews, study subjects participating in respondent validation $(n=4)$ were conveniently selected from the QUAL sample of subjects who completed focus group interviews. Selection of participants was made to provide variety in completed focus group sessions, age, and relationship status. Solicited participants received an explanation of the additional study procedures, criteria for selection of respondent validation participants, confidentiality, and validation guidelines 


\section{Focus Group Interview Guide}

\section{Focus Group Interview Questions}

1. How would you define sexual health?

2. What practices or behaviors do you engage in to be sexually healthy?

3. What behavior(s) do you participate in that prevents you from achieving or maintaining your sexual health?

4. Who contributes to your achieving or maintaining a state of sexual health?

5. What concerns you about your sexual health?

6. How do your concerns help or prevent you from being sexually healthy?

7. Who contributes to the concerns you have about your sexual health? How does this individual(s) contribute to your sexual health?

8. How do you think emotional factors (or issues) contribute to your sexual health?

9. How do you think emotional factors (or issues), such as spirituality or religiosity, depression, depressive symptoms, or mental health, contribute to your sexual health?

10. What other kinds of emotional factors or issues that deal with your feelings contribute to your sexual health?

11. How do you think your intimate relationship(s) contribute to your sexual health?

12. How do you think power or dominance within your intimate relationship(s) contribute to your sexual health?

13. How do other relationships (with friends or family) contribute to your sexual health?

14. How does maintaining your sexual health influence the sexual behaviors that you participate in?

15. What do you feel is the most important issue we've discussed today?

Figure 4-1. Focus Group Interview Guide. 
or procedures. Additional informed consent was obtained during face-to-face private meetings with study participants. Participants completed an individual dissemination session, during which time the study investigator provided participants with a 1-page written report summarizing the investigators' interpretation of qualitative study findings. Participants were then offered the opportunity to comment on the summary of findings. Once dissemination sessions were completed, participants received a retail store gift card.

\section{Quantitative Measures}

Quantitative data were obtained to examine relationships between selected emotional factors (i.e., spirituality, religiosity, and depressive symptomatology) and a relational factor (i.e., gender inequality) derived from the Sexual Health Model and sexual health and risk behaviors in the study sample (Robinson et al., 2002). Six paper and pencil questionnaires were used to quantify each of the factors examined in this study. Instruments used to collect demographic data from study participants and measure sexual health, sexual risk behavior, religiosity, spirituality, depressive symptomology and gender inequality included: Participant Demographic Data Form, Sexual Quality of Life (SQOL-F) Questionnaire (Symonds et al., 2005), Sexual Activities and Attitudes Questionnaire (SAAQ) (Noll et al., 2003), Brief Multidimensional Measure of Religiousness/Spirituality (BMMRS) (Fetzer Institute \& National Institute on Aging Working Group, 1999), Center for Epidemiologic Studies Depression Scale Revised (CESD-R) (Eaton et al., 2004), and the Sexual Relationship Power Scale (SRPS) (Pulerwitz et al., 2000).

Participant demographic data form. An investigator-developed questionnaire (Appendix C) was used to gather pertinent demographic information from study participants related to sexual health and risk behavior. The self-report questionnaire contained distinguishing characteristics. Participants were also questioned about their general health status, by asking them to list current diagnosed medical conditions or diseases, along with family history of specific mental health conditions (i.e., depression, emotional, substance-related, addiction disorders). Lastly, participants were asked to list their religious preference (i.e. Buddhism, Christianity, Islam, Judaism, no religion).

The sexual quality of life -female (SQOL-F) questionnaire. The SQOL-F was developed as a questionnaire to determine the influence of sexual dysfunction on a woman's life satisfaction or quality of life (QOL) (Aalsma et al., 2013; Maasoumi et al., 2013; Symonds et al., 2005). Sexual dysfunction, a concept often used in literature to identify sexual ill-health, has been used in the SQOL-F to recognize aspects of function that impact specific dimensions of QOL (Symonds et al., 2005). The self-report instrument contains 18 items that are scored on a six-point rating scale. The items, which specifically evaluate sexual confidence, emotional well-being, and relationship concerns are totaled, then normalized to a 1 to 100 point scale, and are not used for measuring separate domains (Pfizer Incorporation, 2014; Symonds et al., 2005). Higher total scores 
on the SQOL-F are indicative of a better sexual QOL (Symonds et al., 2005). Factor analysis, tests for internal consistency, convergent validity, and test retest reliability provide substantial evidence to support use of the SQOL-F as an effective measure of sexual health in this study.

The sexual activities and attitudes questionnaire (SAAQ). The SAAQ is a selfreport measure containing 44-items that assess both sexual activities and sexual attitudes (Noll, 2003). Sexual attitudes were examined by asking participants to reflect only upon sexual activities that were engaged in willingly, rather than those that were involuntary or forced (Noll \& Shenk, 2013; Noll et al., 2003). The SAAQ includes seven variables to measure sexual activity. Activities assessed include: 1) age at first voluntary sexual intercourse; 2) birth control efficacy (current); 3) sexual intercourse partners (number within the last year); 4) HIV-risk behaviors (risks include having sexual intercourse: unprotected, while under the influence of drugs or alcohol, with IV drug users, during a "one-night stand); 5) STIs (including gonorrhea, chlamydia, syphilis, genital herpes, genital warts, hepatitis B and C); 6) pregnancies (history, previous birth control efficacy); and 7) sexual behaviors of peers (Noll, 2003). For the purposes of this study, subscale scores for sexual activity and HIV risk behavior were used to determine sexual risk behavior. This subscale score is obtained by having respondents indicate the number of intimate partners with which they have engaged in various behaviors ranging from oral sex to having sexual intercourse while under the influence of drugs or alcohol during their lifetime (Noll, 2003). The possible range of subscale scores is from 0 to 25 , with higher subscale sores indicating participation in riskier sexual behavior throughout one's lifetime. Higher subscale scores for sexual activities and HIV risk behavior are indicative of higher risk related to sexual behavior. Psychometric tests including predictive and discriminant validity confirm the instrument's ability to assess sexual activities in AAW (Noll \& Shenk, 2013; Noll et al., 2003).

The brief multidimensional measure of religiousness / spirituality (BMMRS). The BMMRS is a 38-item, self-report measure designed to distinguish between the concepts of spirituality and religiosity while measuring their connection to health (Fetzer Institute \& National Institute on Aging Working Group, 1999; Johnstone et al., 2012; Johnstone et al., 2009). The instrument was developed under the assumption that religiousness and spirituality are linked to health through behavioral, social, psychological, and physiological factors (Fetzer Institute \& National Institute on Aging Working Group, 1999). The instrument includes 12 domains each selected by developers based upon their theoretical or practical influence on health outcomes (Fetzer Institute \& National Institute on Aging Working Group, 1999; Johnstone et al., 2009; J. Williams et al., 2012). Instrument items vary from being scored on a four- to eight-point rating scale, and also include dichotomous (yes/no) and open ended, short answer questions (Harris et al., 2008).

For the purposes of this study, the Values/Beliefs subscale of the BMMRS was used as a measure of religiosity/spirituality. The Values/Beliefs subscale assesses the 
degree to which an individual's behavior reflects conviction or religion, along with acceptance of religious and/or spiritual views (Fetzer Institute \& National Institute on Aging Working Group, 1999). Lower Values/Beliefs subscale scores (lower than 4) indicate an individual's ability to use religion and/or spirituality to devalue selfstimulation, indulgence, or gratification (Fetzer Institute \& National Institute on Aging Working Group, 1999). The possible range of subscale scores is from 2 to 8. Completed analyses confirm reliability and validity for each of the instrument's domains and support its use in both young and older adults (Fetzer Institute \& National Institute on Aging Working Group, 1999; Johnstone et al., 2012; Johnstone et al., 2009; Radloff, 1977).

The Center for Epidemiologic Studies depression scale revised (CESD-R). The CESD-R is 20-item, self-report measure designed to identify symptoms of depression and depressive disorder (Center for Innovative Public Health Research, 2014; Shim et al., 2013; Van Dam \& Earleywine, 2011). Revised to accommodate modern diagnostic criteria, the CESD-R uses nine groups as defined by the American Psychiatric Association Diagnostic and Statistical Manual, fourth edition (DSM-IV) to distinguish symptoms of depression or depressive disorder (Center for Innovative Public Health Research, 2014; Eaton et al., 2004; Van Dam \& Earleywine, 2011). The nine domains used to assess depressive symptoms include: 1) sadness, 2) loss of interest, 3) appetite, 4) sleep, 5) thinking/concentration, 6) guilt, 7) tired, 8) movement, and 9) suicidal ideation (Center for Innovative Public Health Research, 2014; Eaton et al., 2004). Instrument items assessing the frequency of symptoms in these areas are scored on a 5-point rating scale with possible total scores ranging from 0 to 60 (Eaton et al., 2004). Having a CESD-R total score of less than 16 is determined as not having significant depressive symptoms or a major depressive episode and categorized as not having any clinical significance (Eaton et al., 2004). Tests of internal consistency and convergent and divergent validity in previous studies support use of the CESD-R as an accurate measure of depressive symptomology in this study. For study purposes, total scores on the CESD$\mathrm{R}$ were used as a measure of depressive symptomology.

The sexual relationship power scale (SRPS). The SRPS, developed by Pulerwitz, Gortmaker, and DeJong, is a 23-item measure of issues occurring within relationships (relationship dynamics) that contain two domains or subscales of relationship power including 1) relationship control and 2) decision-making dominance (Matsuda et al., 2012; Pulerwitz et al., 2000). The relationship control subscale (15 items) focuses on questions related to a participant's perception of her partner's control, while the decision-making dominance subscale ( 8 items) asks questions about the partner having greater influence on decisions made in the participant's every life (Matsuda et al., 2012; Pulerwitz et al., 2000). By using a 4-point (relationship control subscale) and 3point (decision-making dominance) rating scale, both subscale and total scores are used to determine categories that represent low, medium, and high levels of relationship power. The possible range of subscale scores for Relationship Control is from 15 to 60 and 8 to 24 for Decision-making Dominance. The possible range of total scores is from 23 to 84 , with higher total sores indicating participants' perception of having greater 
power within their sexual relationships (Matsuda et al., 2012; Pulerwitz et al., 2000). Psychometric testing including content, face, and construct validity support use of this instrument as a valid and reliable measure of relationship power for these women (Pulerwitz et al., 2000). For the purposes of this study, both subscale and total scores on the SRPS were used as measures of gender inequality.

\section{Quantitative Analysis and Results}

Quantitative data collected from study participants were analyzed using Statistical Analysis Software (SAS) version.9.4 (SAS Institute Inc., Cary NC). Statistical significance was set at an alpha of .05. Initially, descriptive statistics were used to characterize the study's sample using counts and frequencies for categorical variables and means and standard deviations for continuous variables. Instrument scores were also summarized using mean and standard deviation distributions. Reliability of instruments was estimated using Cronbach's $\alpha$ based on the current study sample and compared to previously reported results of reliability. Correlational analysis using Pearson product moment correlation coefficients was completed to estimate the direction and magnitude of relationships among emotional and relational variables and outcome variables (sexual risk behavior). Both simple and multiple linear regression analyses were then used to assess whether selected emotional and relational factors predict either study outcomes. Potential predictors with associated p-values less than .20 were identified as candidate variables for inclusion in multiple regression models.

Standard multiple linear regression analysis was used to determine whether specified emotional and relational factors influenced women's sexual health and sexual risk behavior. Regression models were fitted using significant variables identified during correlation analysis. Additionally, demographic variables were tested for inclusion in regression models, by adding age, relationship status, and education level.

\section{Participants}

Study participants were 50 self-identified, adult, AAW residing within a large, urban community. Additional criteria for study inclusion restricted the participants to women who reported engaging in sexual activity within the past 12 months. Sexual activity in this study was defined as oral (oral-to-genital contact), vaginal (penetration of the vagina), or anal (penetration of the anus) intercourse. Women who were actively attempting to become pregnant or who reported being pregnant during study enrollment were excluded. Most of these women reported their sexual orientation as heterosexual $(98 \%)$ or bisexual $(2 \%)$. For this study, bisexual orientation was defined as having a sexual attraction to and/or sexual experiences with both men and women. Ages of study participants ranged from 21 to 64 years $(\mathrm{M}=38.5, \mathrm{SD} \pm 11.35)$. Over half of study participants were single $(44 \%)$ or divorced $(10 \%)$. Further demographic information is summarized in Table 4-1. 
Table 4-1. Demographic Characteristics as Reported by a Sample of African American Women.

\begin{tabular}{lcc}
\multicolumn{1}{c}{ Characteristics } & $\begin{array}{c}\text { QUAN Sample }(\mathrm{N}=50) \\
\mathrm{n}(\%)\end{array}$ & $\begin{array}{c}\text { QUAL Sample }(\mathrm{n}=19) \\
\mathrm{n}(\%)\end{array}$ \\
\hline Relationship Status & $22(44)$ & $7(36.84)$ \\
Single & $19(38)$ & $8(42.11)$ \\
Married & $5(10)$ & $210.53)$ \\
Divorced & $4(8)$ & $2(10.53)$ \\
Living with Partner & & \\
Highest Education Level & $2(4)$ & $1(5.26)$ \\
Grade 9 - 12 & $11(22)$ & $6(31.58)$ \\
High School or GED & $22(44)$ & $5(26.32)$ \\
2 or 4 Year College & $15(30)$ & $7(36.84)$ \\
Post-Graduate College & & \\
Employment Status & $44(88)$ & $17(89.47)$ \\
Formally Employed & $2(4)$ & $1(5.26)$ \\
Self-Employed & $4(8)$ & $1(5.26)$ \\
Unemployed & & \\
Health Insurance Coverage & $40(80)$ & $17(89.47)$ \\
Private/Employer & $4(8)$ & \\
Provided & & $0(10.53)$ \\
Government Provided - & $1(2)$ & $0(0)$ \\
Obamacare & $2(4)$ & $0(0)$ \\
Unknown & $3(6)$ & \\
Medicare/Tricare & Uninsured &
\end{tabular}

Note. QUAN sample $(\mathrm{N}=50)$ reflects the full study sample completing the study's quantitative component. QUAL sample $(n=19)$ reflects the subsample of QUAN participants completing the study's qualitative component. Education level reported reflects the highest level completed. 
Characteristics describing the sexual health (Table 4-2) of study participants indicate that most of these women were pre-menopausal (68\%). Pre-menopausal was defined in this study as having regular monthly menstrual cycles. Most participants sought reproductive health care within the past year (72\%) with slightly over half using a pregnancy prevention method or device (52\%).

Although very few participants reported tobacco and illicit drug use (6\%), most (70\%) indicated consumption of alcohol (Table 4-3). Alcohol intake ranged from not having any alcoholic beverages to consuming two alcoholic beverages per week $(\mathrm{M}=$ $1.68 ; \mathrm{SD}=2.8$ ). Tobacco use among study participants varied from not smoking to smoking 1 pack of tobacco products per day $(\mathrm{M}=.05 ; \mathrm{SD} \pm .2$ i.e., 1 cigarette/day \pm 4 cigarettes). All illicit drug use reported by participants involved the use of marijuana.

General health problems (Table 4-4) most commonly reported by participants included hypertension $(20 \%)$ and asthma (14\%). The most commonly reported mental health conditions were depression (12\%) and anxiety (10\%). Participants most frequently reported having a history of both conditions in their families with $18 \%$ reporting having a family history of depression and 12\% reporting a family history of anxiety (Table 4-4).

\section{Sexual Health, Sexual Risk Behavior, Depressive Symptomology, Spiritualty / Religiosity, and Gender Inequality Scores}

Self-assessment scores for sexual health, sexual risk behavior, spirituality/religiosity, depressive symptomology, and gender inequality are summarized in Table 4-5. Each scale and subscale used to measure variables examined in this study are based on the possible range of scores generated from rating participants' responses and/or norm-referenced by comparing scores to a sample of healthy women.

Sexual health. The SQOL-F demonstrated good internal consistency in the current study as a reliable measure of sexual health with Cronbach's alpha of .82. Study participants had a mean score of slightly above $90(\mathrm{M}=90.28, \mathrm{SD}= \pm 12.4)$ indicating a good overall sexual quality of life. Average total scores for a sample of healthy women is 90.1 (Symonds et al., 2005).

Sexual risk behavior. The sexual risk behavior score is obtained from a subscale of the Sexual Activities and Attitudes Questionnaire (SAAQ) (Noll, 2003). In the current study the risky sexual behavior subscale demonstrated good internal consistency as a reliable measure of sexual risk behavior with Cronbach's alpha of .79. Study participants had a mean sexual risk behavior score of $10.12(\mathrm{SD}= \pm 5.2)$, indicating participation in relatively lower risk behavior (Bouchard et al., 2009). 
Table 4-2. Sexual Health Characteristics as Reported by a Sample of African American Women.

\begin{tabular}{|c|c|c|}
\hline Characteristics & $\begin{array}{c}\text { QUAN Sample }(\mathrm{N}=50) \\
\mathrm{n}(\%)\end{array}$ & $\begin{array}{c}\text { QUAL Sample }(\mathrm{n}=19) \\
\mathrm{n}(\%)\end{array}$ \\
\hline \multicolumn{3}{|l|}{ Reproductive Health Status } \\
\hline Pre-menopausal & $34(68)$ & $12(63.16)$ \\
\hline Perimenopausal & $3(6)$ & $2(10.52)$ \\
\hline Post-menopausal & $9(18)$ & $3(15.79)$ \\
\hline Unknown & $4(8)$ & $2(10.53)$ \\
\hline \multicolumn{3}{|l|}{ Last Reproductive Health } \\
\hline \multicolumn{3}{|l|}{ Care Visit } \\
\hline Less than 1 year & $36(72)$ & $16(84.21)$ \\
\hline $2-3$ years & $10(20)$ & $3(15.79)$ \\
\hline $4-5$ years & $1(2)$ & $0(0)$ \\
\hline Over 5 years & $3(6)$ & $0(0)$ \\
\hline \multicolumn{3}{|l|}{ Contraceptive Use } \\
\hline Birth Control Pill & $9(18)$ & $3(15.79)$ \\
\hline Birth Control Implant & $2(4)$ & $1(5.26)$ \\
\hline Vaginal Ring & $1(2)$ & $0(0)$ \\
\hline Intrauterine Device (IUD) & $1(2)$ & $0(0)$ \\
\hline Male Condom & $10(20)$ & $5(26.32)$ \\
\hline Withdrawal & $4(8)$ & $1(5.26)$ \\
\hline None & $22(44)$ & $9(47.37)$ \\
\hline Pill \& Male Condom & $1(2)$ & $0(0)$ \\
\hline
\end{tabular}

Note. QUAN sample $(\mathrm{N}=50)$ reflects the full study sample completing the study's quantitative component. QUAL sample $(\mathrm{n}=19)$ reflects the subsample of QUAN participants completing the study's qualitative component. In this study, pre-menopausal was defined as having regular menstrual cycles, perimenopausal was defined as having irregular menstrual cycles, and post-menopausal was defined as 12 months without a menstrual cycle. 
Table 4-3. Substance Use as Reported by a Sample of African American Women.

\begin{tabular}{lcc}
\hline \multicolumn{1}{c}{ Characteristics } & $\begin{array}{c}\text { QUAN Sample }(\mathrm{N}=50) \\
\mathrm{n}(\%)\end{array}$ & $\begin{array}{c}\text { QUAL Sample }(\mathrm{n}=19) \\
\mathrm{n}(\%)\end{array}$ \\
\hline $\begin{array}{l}\text { Alcohol Use } \\
\text { None }\end{array}$ & $15(30)$ & $4(21.06)$ \\
Yes & $35(70)$ & $15(78.95)$ \\
Tobacco Use & $47(94)$ & $19(100)$ \\
None & $3(6)$ & $0(0)$ \\
Yes & $47(94)$ & $18(94.74)$ \\
Illicit/Illegal Drug Use & $3(6)$ & $1(5.26)$ \\
$\quad$ None & $\begin{array}{l}\text { Yes } \\
\text { Note. QUAN sample }(\mathrm{N}=50) \text { reflects the full study sample completing the study's } \\
\text { quantitative component. QUAL sample (n=19) reflects the subsample of QUAN } \\
\text { participants completing the study's qualitative component. }\end{array}$
\end{tabular}


Table 4-4. General and Mental Health Characteristics as Reported by a Sample of African American Women.

\begin{tabular}{lcc}
\hline \multicolumn{1}{c}{ Characteristics } & $\begin{array}{c}\text { QUAN Sample }(\mathrm{N}=50) \\
\mathrm{n}(\%)\end{array}$ & $\begin{array}{c}\text { QUAL Sample }(\mathrm{n}=19) \\
\mathrm{n}(\%)\end{array}$ \\
\hline General Health Conditions & & \\
Hypertension & $10(20)$ & $4(21.05)$ \\
Diabetes & $1(2)$ & $1(5.26)$ \\
Asthma & $7(14)$ & $1(5.26)$ \\
Vision/Hearing Disorders & $4(8)$ & $1(5.26)$ \\
Musculoskeletal Disorders & $4(8)$ & $1(5.26)$ \\
Mental Illness/Mental Health & & \\
Conditions & $6(12)$ & $4(21.05)$ \\
Depression & $1(2)$ & $1(5.26)$ \\
Bipolar Disorder & $5(10)$ & $2(10.53)$ \\
Anxiety Disorder & & \\
Family History Mental & & \\
Illness/Mental Health & & \\
Conditions & $9(18)$ & $2(21.05)$ \\
Depression & $4(8)$ & $3(10.53)$ \\
Bipolar Disorder & $6(12)$ & $2(10.53)$ \\
Anxiety Disorder & $3(6)$ & \\
Schizophrenia & & \\
\hline
\end{tabular}

Note. QUAN sample $(\mathrm{N}=50)$ reflects the full study sample completing the study's quantitative component. QUAL sample $(\mathrm{n}=19)$ reflects the subsample of QUAN participants completing the study's qualitative component. 
Table 4-5. Self-Reported Sexual Health, Risk Behavior, Emotional and Relational Factors as Reported by a Sample of 50 African American Women.

\begin{tabular}{|c|c|c|c|}
\hline Instruments & Variable Measured & $\mathrm{M} \pm \mathrm{SD}$ & $\begin{array}{l}\text { Possible Range of } \\
\text { Scores }\end{array}$ \\
\hline Sexual Quality of Life Questionnaire Total Score & Sexual Health & $90.28 \pm 12.4$ & $18-108$ \\
\hline $\begin{array}{l}\text { Sexual Activities and Attitudes Questionnaire Sexual } \\
\text { Risk Behavior Subscale Score }\end{array}$ & Sexual Risk Behavior & $10.12 \pm 5.2$ & $0-25$ \\
\hline $\begin{array}{l}\text { Centers for Epidemiological Studies Depression } \\
\text { Scale - Revised Total Score }\end{array}$ & Depressive Symptomology & $8.38 \pm 9.8$ & $0-60$ \\
\hline $\begin{array}{l}\text { Brief Multidimensional Scale of Religiousness and } \\
\text { Spirituality Values/Beliefs Subscale Score }\end{array}$ & Religiosity/Spirituality & $2.9 \pm 1$ & $2-8$ \\
\hline Self-Ranking: Religiousness Subscale Score & & $2.04 \pm 0.8$ & $1-4$ \\
\hline Self-Ranking: Spiritual Subscale Score & & $1.6 \pm 0.7$ & $1-4$ \\
\hline Sexual Relationship Power Scale Total Score & Gender Inequality & $70.54 \pm 7.2$ & $23-84$ \\
\hline Relationship Control Subscale Score & & $53.54 \pm 5.7$ & $15-60$ \\
\hline Decision Making Dominance Subscale Score & & $17 \pm 2.6$ & $8-24$ \\
\hline
\end{tabular}


Depressive symptomology. In the current study, the CESD-R demonstrated excellent internal consistency as a reliable measure of depressive symptomology with Cronbach's alpha of .92. Study participants had a mean score of slightly above $8(\mathrm{M}=$ $8.38, \mathrm{SD}=9.8$ ) indicating the presence of few depressive symptoms. While participants had a low mean CESD-R score, the range of reported scores was from 0 to 43. These results reflect only three participants with scores above 35 . Instrument scores at or above this level may be categorized as probable or meeting the criteria for experiencing a major depressive episode (Eaton et al., 2004).

Spirituality / religiosity. The spirituality and religiosity scores are obtained from a subscale of the BMMRS (Fetzer Institute \& National Institute on Aging Working Group, 1999). Study participants had a mean score of $2.9(\mathrm{SD}= \pm 1)$, indicating an orientation to engage in behaviors that are encouraged by one's faith. Average Values/Beliefs subscale scores for a healthy sample of both men and women is 3.08 (Fetzer Institute \& National Institute on Aging Working Group, 1999).

Gender inequality. In the current study, the SRPS demonstrated good internal consistency as a reliable measure of gender inequality with Cronbach's alpha of .85. Study participants had mean Relationship Control and Decision-making Dominance subscale scores of $53.54(\mathrm{SD}= \pm 5.7)$ and $17(\mathrm{SD}= \pm 2.6)$, respectively, both revealing high levels of control and dominance while making decisions within their relationships. The mean total score for study participants was $70.54(\mathrm{SD}= \pm 7.2)$ indicating a high level of overall relationship power.

\section{Relationships among Sexual Health, Sexual Risk Behavior, Depressive Symptomology, and Gender Inequality}

Table 4-6 displays estimated Pearson product moment correlation coefficients among emotional and relational factors and both outcome variables, sexual health and sexual risk behavior; those with associated p-values less than .20 are indicated as candidate variables for inclusion in multiple regression models. Variation in these women's depressive symptoms and gender inequality were correlated with sexual health or participation in sexual risk behaviors $(p=0.20 ; p=0.08 ; p=0.18 ; p=0.19 ; p=0.11$; $\mathrm{p}=0.08 ; \mathrm{p}=0.17$ ).

Both depressive symptomology and decision-making dominance were negatively associated with sexual health $(r=-.19 ; p<.20 ; r=-0.25 ; p<.10$, respectively). Decision-making dominance was positively associated with sexual risk behavior $(r=$ $0.19 ; p<.20)$. The association between religiosity/spirituality and both outcome variables were not statistically significant. 
Table 4-6. Relationships among Sexual Health, Sexual Risk Behavior, Depressive Symptomology, and Gender Inequality as Reported by a Sample of 50 African American Women.

\begin{tabular}{lcccc}
\hline \multicolumn{1}{c}{ Variable } & SQOL-F & SAAQ & CESD-R & SRPS \\
\hline SQOL-F & - & $-.28^{*}$ & $-.19^{*}$ & $-.25^{*}$ \\
SAAQ & $-.28^{*}$ & - & .09 & $.19^{*}$ \\
CESD-R & $-.19^{*}$ & .09 & - & $-.22^{*}$ \\
SRPS & $-.25^{*}$ & $.19^{*}$ & $-.22^{*}$ & -
\end{tabular}

Note. Estimated Pearson product-moment correlation coefficients. Significance for identification of candidate predictors was set a priori at $\mathrm{p} \leq .20$. SQOL-F $=$ Sexual Quality of Life Questionnaire - Female total score; SAAQ = Sexual Activities and Attitudes Questionnaire sexual activities subscale score; CESD-R = Center for Epidemiologic Studies Depression Scale - Revised total score; SRPS = Sexual Relationship Power Scale Decision-Making Dominance subscale score.

$* \mathrm{p}<.20$ 
Standard linear regression was conducted to determine the influence of decisionmaking dominance did not influence women's sexual risk behavior $\left(\mathrm{R}^{2}=0.0358, \mathrm{p}=\right.$ 0.1884 ). Multiple linear regression analysis was used to determine the influence of decision-making dominance and depressive symptomology on sexual health (Table 4-7). The overall model explained $12 \%$ of the variance in women's sexual health $(\mathrm{R} 2=$ $0.1211, \mathrm{p}>0.05$ ); however depressive symptomology was not significant, indicating that the presence of depressive symptoms did not independently influence participants' sexual health after adjusting for decision-making dominance.

With reported scores for depressive symptomology varying from scores indicating the experience of little to no symptoms to those suggesting significant depressive symptoms, consideration was given to the connection of depressive symptomology with other variables examined in this study. In this study, relationships among sexual health, sexual risk behavior, depressive symptomology, and gender inequality may have been influenced by those participants who experienced significantly higher levels of depressive symptoms rather than most study participants. Examination of these women's depressive symptoms revealed three outliers, though their presence did not affect assumption for normality. In an effort to ensure that impressions generated based upon participants' depressive symptoms scores were not driven by these outliers, participant data was analyzed after removing outliers or participants with CESD-R scores greater than 35 from the full study sample.

Self-assessment scores for sexual health, sexual risk behavior, spirituality/religiosity, depressive symptomology, and gender inequality are summarized for the study's subsample $(n=47)$ in Table 4-8. After removing the identified outliers, mean CESD-R scores reported for the subsample of women were nearly two points lower $($ CESD-R mean score $=6.5)$ than that of the entire study sample (CESD-R mean score $=$ 8.4). Table 4-9 displays estimated Pearson product moment correlation coefficients among emotional and relational factors and both outcome variables, sexual health and sexual risk behavior $(\mathrm{p}<.20)$ for the subsample of study participants with CESD-R scores lower than 35 . Similar to the full study sample, after eliminating the women who experienced increased symptoms of depression, variation in depressive symptoms and decision-making dominance were negatively correlated with sexual health $(r=-.24 ; p<$ $.20 ; r=-.26 ; p<.10$, respectively). Additionally, with the removal of outliers, depressive symptomology and decision-making dominance were both correlated with sexual risk behavior $(\mathrm{r}=.20 ; \mathrm{p}<.20 ; \mathrm{r}=.20 ; \mathrm{p}<.20$, respectively). Based on this subsample of participants, the multiple linear regression model fit including decision-making dominance and depressive symptomology did not significantly predict women's sexual health or risk behavior.

\section{Qualitative Analysis}

Qualitative data were collected and analyzed to enhance and/or refine quantitative findings. Qualitative data were analyzed using an inductive approach whereby patterns 
Table 4-7. Multiple Regression Model Predicting Sexual Health for a Sample of 50 African American Women.

\begin{tabular}{lccc}
\hline \multicolumn{1}{c}{ Variable } & $b$ & $S E$ & $p$ \\
\hline 1. Decision-making Dominance & -1.43 & 0.67 & 0.04 \\
2. Depressive Symptomology & -0.32 & 0.18 & 0.08 \\
\hline
\end{tabular}

Note. $\mathrm{R}^{2}=0.12 ; p<.05$ 
Table 4-8. $\quad$ Self-Reported Sexual Health, Risk Behavior, Emotional and Relational Factors as Reported by a Sample of 47 African American Women.

\begin{tabular}{|c|c|c|c|}
\hline Instruments & Variable Measured & $\mathrm{M} \pm \mathrm{SD}$ & $\begin{array}{l}\text { Possible Range of } \\
\text { Scores }\end{array}$ \\
\hline Sexual Quality of Life Questionnaire Total Scale & Sexual Health & $90.49 \pm 12.6$ & $18-108$ \\
\hline $\begin{array}{l}\text { Sexual Activities and Attitudes Questionnaire Sexual } \\
\text { Risk Behavior Subscale Score }\end{array}$ & Sexual Risk Behavior & $10.19 \pm 5.2$ & $0-25$ \\
\hline $\begin{array}{l}\text { Centers for Epidemiological Studies Depression } \\
\text { Scale - Revised Total Scale }\end{array}$ & Depressive Symptomology & $6.47 \pm 6.3$ & $0-60$ \\
\hline $\begin{array}{l}\text { Brief Multidimensional Scale of Religiousness and } \\
\text { Spirituality Values/Beliefs Subscale Score }\end{array}$ & Religiosity/Spirituality & $2.91 \pm 1$ & $2-8$ \\
\hline Self-Ranking: Religiousness Subscale Score & & $2.09 \pm 0.8$ & $1-4$ \\
\hline Self-Ranking: Spiritual Subscale Score & & $1.62 \pm 0.7$ & $1-4$ \\
\hline Sexual Relationship Power Scale Total Score & Gender Inequality & $71.40 \pm 6.5$ & $23-84$ \\
\hline Relationship Control Subscale Score & & $54.2 \pm 5.2$ & $15-60$ \\
\hline Decision Making Dominance Subscale Score & & $17.21 \pm 2.5$ & $8-24$ \\
\hline
\end{tabular}


Table 4-9. Relationships among Sexual Health, Sexual Risk Behavior, Depressive Symptomology, and Gender Inequality as Reported by a Sample of 47 African American Women.

\begin{tabular}{lcccc}
\hline \multicolumn{1}{c}{ Variable } & SQOL-F & SAAQ & CESD-R & SRPS \\
\hline SQOL-F & - & $-.29^{*}$ & $-.24^{*}$ & $-.26^{*}$ \\
SAAQ & $-.29^{*}$ & - & $.20^{*}$ & $.20^{*}$ \\
CESD-R & $-.24^{*}$ & $.20^{*}$ & - & .09 \\
SRPS & $-.26^{*}$ & $.20^{*}$ & .09 & - \\
\hline
\end{tabular}

Note. Estimated Pearson product-moment correlation coefficients without three outliers based on Centers for Epidemiological Studies Depression Scale - Revised scores (greater than 35). Significance was set a priori at $\mathrm{p} \leq .20$. SQOL-F $=$ Sexual Quality of Life Questionnaire - Female total score; SAAQ $=$ Sexual Activities and Attitudes Questionnaire sexual activities subscale score; CESD-R = Center for Epidemiologic Studies Depression Scale - Revised total score; SRPS = Sexual Relationship Power Scale Decision-Making Dominance subscale score.

$* \mathrm{p}<.20$ 
were identified and theory derived from the Sexual Health Model validated directly from data collected during focus group interviews. Initially, portions or segments of focus group transcripts were examined to allow for summarization of participants' responses. Interview transcripts were then entered into NVivo 10 software (QSR International [Americas], Burlington, MA) for thematic content analysis. Data were reduced by coding, a process involving the break down and comparison of concepts presented from participants' experiences related to sexual health. Codes were then clustered into categories by themes emerging from the categories. Coding completed by the study investigator was discussed and validated with members of the research team. Final themes generated from the completion of multiple comparisons of identified codes were used to describe participants' perception of sexual health.

To ensure the credibility of findings deduced from QUAL analysis, individual dissemination sessions were carried out with selected focus group participants $(n=4)$ for respondent validation (Green \& Thorogood, 2009). Respondent validation, or member checking, was conducted with the intent of seeking participants' corroboration or disapproval of whether investigators' interpretation accurately reflected the thoughts and feelings expressed during focus group interviews (Silverman, 2011). After collaborative, refining discussion, participants confirmed the accuracy of final QUAL themes, associated definitions, and illuminating quotes. Participants' validation also generated discussion regarding further exploration of sexual health and risk behavior for AAW. One participant suggested an additional study to only examine "the new normal" by looking at specific factors. One factor suggested was having sexual or intimate relationships with "men on the down low", or men who do not openly disclose their preference to have sex with other men while maintaining relationships with women.

\section{Qualitative Results}

\section{Participants}

Qualitative study participants included 19 women each of whom met the study's inclusion criteria and previously completed the study's quantitative component. The QUAL subsample reported their sexual orientation as heterosexual (94.7\%) or bisexual (5.3\%). Age ranges for QUAL participants were from 25 to 64 years $(\mathrm{M}=41.1, \mathrm{SD} \pm$ $10.25)$. Nearly half of study participants were single $(36.9 \%)$ or divorced $(10.5 \%)$. Further demographic information is summarized in Table 4-1.

Characteristics describing the sexual health (Table 4-2) of the study's QUAL sample indicate that most of these women were pre-menopausal (63.2\%). Much like the QUAN sample, most participants sought reproductive health care within the past year $(84.2 \%)$ with nearly half using a pregnancy prevention method or device $(47 \%)$. 
Reports of substance use among the study's QUAL sample indicate no tobacco use with very few reporting illicit drug use (5.2\%). Illicit drug use reported was the use of marijuana. Most of these women (79\%) indicated consumption of alcohol

(Table 4-3). Alcohol intake ranged from not having any alcoholic beverages to consuming 14 alcoholic beverages per week $(\mathrm{M}=2.7 ; \mathrm{SD}=4.1)$.

General health problems (Table 4-4) most commonly reported by QUAL participants included hypertension (21.1\%). The most commonly reported mental health conditions were depression $(21.1 \%)$ and anxiety (10.5\%). Participants most frequently reported having a history of both conditions in their families with $21.05 \%$ reporting having a family history of depression and $15.8 \%$ reporting a family history of anxiety (Table 4-4).

\section{Perceptions of Sexual Health}

Participants' perception of sexual health was explored using thematic content analysis. Recurring patterns from both focus group interviews were interpreted and organized by category into over-arching themes. Each identified code and category generated during QUAL data analysis was defined specifically for the purposes of this study (Appendix D). Organizing themes that emerged from QUAL data included: 1) "The new normal"; 2) having to compromise; 3) taking responsibility; 4) securing an attachment; and 5) resolving ambiguity. Table 4-10 depicts thematic content analyses conducted which revealed final themes, some of which emerged from the same categories.

\section{The New Normal}

Participants described experiences of their current sexual climate, acknowledging the influence of their peers and society in shaping "the new normal". Both peers and society were acknowledged as having an important influence on acquiring or maintaining a desired state of sexual health. The theme, "the new normal" emerged from three recurring categories identified throughout focus group interviews. The first, assimilation, was defined in this study as women's familiarization or adjustment to their sexual culture, well-being, or level of sexual confidence. Secondly, expression, is a category symbolizing participation in various behaviors that express an acknowledgment of sexual health. And, influence was defined in this study as a determinant factor that may serve to guide or alter an individual's sexual decision-making or behavior. Together these categories comprise the "the new normal" for study participants. During interview sessions, these women often referred to the conditions surrounding their sexuality, sexual behaviors, and what may be deemed acceptable as, "the new normal".

Assimilation was conveyed in participants' familiarization or adjustment to "the new normal". This adjustment was also recognized as vital to achieving an optimal level of sexual well-being. Adequately addressing sexual health for study participants often 
Table 4-10. Thematic Structure of Perceptions of Sexual Health for a Sample of African American Women $(n=19)$.

\begin{tabular}{|c|c|c|}
\hline Themes & Categories & Codes \\
\hline "The New Normal" & $\begin{array}{l}\text { Assimilation; } \\
\text { Expression; } \\
\text { Influence }\end{array}$ & $\begin{array}{l}\text { Essential, Developing } \\
\text { Awareness; Generational } \\
\text { Approach; Family-Work } \\
\text { Demands }\end{array}$ \\
\hline Having to Compromise & $\begin{array}{l}\text { Multi-dimensional; } \\
\text { Self-sufficiency; } \\
\text { Relational; } \\
\text { Sexual }\end{array}$ & $\begin{array}{l}\text { Holistic, Mental vs. } \\
\text { Physical; } \\
\text { Decision-making; } \\
\text { Personal Standards, Self- } \\
\text { concept; Comfort }\end{array}$ \\
\hline Taking Responsibility & $\begin{array}{l}\text { Assimilation } \\
\text { Relational } \\
\text { Self-sufficiency }\end{array}$ & $\begin{array}{l}\text { Developing, Awareness; } \\
\text { Relationship Dynamics, } \\
\text { Fidelity } \\
\text { Decision-making, } \\
\text { Personal Standards, Self- } \\
\text { concept, Comfort }\end{array}$ \\
\hline Securing an Attachment & $\begin{array}{l}\text { Religiosity-Spirituality } \\
\text { Relational } \\
\text { Emotional } \\
\text { Sexual }\end{array}$ & $\begin{array}{l}\text { Relationship Dynamics, } \\
\text { Fidelity; } \\
\text { Depression/depressive } \\
\text { symptoms, Feelings } \\
\text { Partner's Feelings; } \\
\text { Behaviors/Intercourse, } \\
\text { Emotional Barriers, } \\
\text { Physical Barriers }\end{array}$ \\
\hline Resolving Ambiguity & $\begin{array}{l}\text { Assimilation } \\
\text { Relational } \\
\text { Self-sufficiency }\end{array}$ & $\begin{array}{l}\text { Essential, Developing, } \\
\text { Awareness; } \\
\text { Relationship Dynamics, } \\
\text { Fidelity; } \\
\text { Decision-making, } \\
\text { Personal Standards/Ideals, } \\
\text { Self-Concept, Comfort }\end{array}$ \\
\hline
\end{tabular}


required making transitions that involved embracing alternative sexual lifestyles, modes of communication (i.e., text messaging, video telephony), and various behaviors. One

participant described the experience as: “.... and I'm thinking to myself, you're way out of the loop of what we have going on (laughter) and I'm speaking about even the texting thing. So, we can't just talk anymore?" Another stated: "It's like we have to get used to what is out there in front of us now, whether we accept it or not. It's the new normal. So, either you live with it or you don't. You either accept it or let them live their lives... The different sexuality, it's the new normal...those who don't accept it, don't throw stones because you may be living in a glasshouse, too. You don't want stones thrown at you."

During discussion of "the new normal" focus was often placed on the influences contributing to these women's sexual health and potential risk. This participant noted that there are many factors including friends and society that influence sexual health, by stating: "... but it may be friends, talking 'I would or wouldn't do' or 'you should or shouldn't do'. You have TV that's constantly, you know, parading different things that are acceptable... and, I use that loosely. I think there are a lot of internal and external factors that contribute to sexual health."

Differences in age group and/or life stage also appeared to dictate "the new normal" for study participants. Participants linked generational acceptance of "the new normal" for younger women, to having casual standards or relaxed morals. One participant stated, "It don't take much for the younger generation and I hate that for our young black females". For older participants, family and work demands were cited as key contributors in accepting "the new normal". However, a commonality for women of all ages was recognition of "the new normal" as a developing and evolving component of "just overall well-being".

These findings reveal that these women's sexual health and behavior may be influenced by generational and/or societal factors. The values and beliefs regarding sex that are embraced by one's culture may dictate how these women accept and express their own sexuality. Participants' perception of the current sexual climate and the factors involved in how they adjust to and behave within their environment emerged as their "new normal".

\section{Having to Compromise}

Having to compromise is a theme represented by the participants' obligation to negotiate between several aspects of their sexual health. This theme arises from four categories including: multidimensional, self-sufficiency, relational, and sexual. Multidimensional, a category perceived as significantly contributing to this theme, is recognized in this study as a complex concept composed of or influenced by multiple factors including: physical, emotional, relational, and/or sexual components. Selfsufficiency is a category recognized in this study as factors contributing to or characterizing one's independence and reliance on self for personal sustainability. Additionally, relational is defined as instinctive factors developed by or contributing to 
the connection between an individual and another person. And, sexual is defined in this study as instinctive factors developed by or contributing to one's sexuality and/or the physical connection between an individual and their intimate partner. During focus group discussion, participants illustrated the contribution each of these categories made to the compromises they were faced with.

Participants frequently described the multidimensional aspects of sexual health. For example, sexual health was depicted by participants as merely one dimension of their overall well-being. In one participant's explanation, sexual health was referred to as, “... not just a physical thing, it's mental, and it's emotional'. Achieving a state of balance between the physical, emotional, and sexual components of participants' health was identified as a consistent source of compromise. Examples of participants' compromise to achieve balance toward sexual health include: "Am I trying to maintain my mental health or more of the mental aspect of sex or more of the physical aspect? ... I'm trying to please myself and my partner. So, I throw the physical aspect out the door." "And you shouldn't have to compromise either: --- do you compromise one aspect of your sexual health for the other or is there a way to integrate everything so that you're happy and everyone else is happy?"

In other instances, these women illustrated experiences of feeling compelled to accept less than desirable standards when addressing their independence and intimate or sexual relationships. Compromise was noted to have a significant role in these women's ability to rely on themselves for personal sustainability. Instability in these women's independence was also found to play an equally important part in sustaining or achieving balance in their personal relationships. One participant described the compromise between self-sufficiency, her relationship, and engaging in sexual activity by stating: "I felt like I gave too much of myself and not really communicating to him, to where I'm saying 'look this is where I'm at right now. This is not what I want to do. I can't just really engage with you right now."'

Participants described other negotiation in meeting personal sexual needs. For some of these women, concessions had to be made sexually to maintain other aspects of their reproductive health. "I wanted to be out there sexually but thinking about STIs and HIV, and pregnancy, I'm like 'ugh-ugh! I'm not going to do that!'”

These findings reveal that agreement between multiple factors must be considered to attain an optimal state of sexual well-being for some women. Participants' negotiation in addressing the physical, emotional, relational, and sexual factors contributing to their sexual health served as a source of constant struggle. Having to decide between meeting their mental versus their physical needs and maintaining independence within their relationships often require these women to compromise various aspects of their sexual health. 


\section{Taking Responsibility}

An accountability to both self and sexual or intimate partners was identified by study participants as essential in their accounts of sexual health and emerged as the organizing theme of "taking responsibility". This theme arises from three categories including: assimilation, relational, and self-sufficiency. From this perspective, assimilation focused on these women's familiarization or adjustment to their well-being and level of sexual self-confidence. Relational maintained its focus on those instinctive factors developed by or contributing to the connection between an individual and another person. And, self-sufficiency continued to recognize factors contributing to or characterizing one's independence and reliance on self for personal sustainability. The combination of these categories shaped these women's assumption of responsibility in achieving or maintaining a desired level of sexual health.

Assimilation was depicted in these women's ability to make decisions regarding their sexual health and/or overall well-being. Taking responsibility, for many participants involved making decisions that allowed them to achieve a desired level of well-being and sustain sexual self-confidence within their environment. The ability to make decisions was found to facilitate assumption of responsibility in making necessary adjustments toward maintaining or even recognizing sexual health as an important lifestyle factor. Participants illustrated the gravity of making the right decisions in taking responsibility for their sexual health: "... just being mindful in making healthy decisions, just making wise health decisions... Sometimes, we think we're all intellectual on some level, then you find yourself like "okay, so now why am I doing this?" "Making bad choices, you know --- that's the bottom line as it relates to sexual health."

Self-sufficiency or having an obligation to one's self was described by focus group participants as a primary motivator for actions taken to acknowledge sexual health and behavior. Personal responsibility involved being self-reliant, self-aware, and satisfied with themselves both physically and sexually. Assuming personal responsibility for many participants was described as a necessary provision in both their sexual and intimate relationships: "It has to start with yourself. Nobody is going to take care of you like you. Period." “...being accountable to oneself just like I would be accountable for my mental, my physical. I would go to the doctor, you know, keep uh, appointments, and make sure I'm doing okay in other areas. So, that would be an accountability to me to you know, take care of myself in that aspect as well." "... it's about knowing your body. What makes your body tick, knowing what makes your body ache, what makes your body ail, uh, knowing with your body when something is not right with it." "...it's like are you comfortable with yourself? Like, do you even feel comfortable about the subject? Do you feel comfortable talking with someone (about it) because you can't have a healthy sexual relationship with somebody if you ... don't feel good about --- (you)."

The category relational was represented by participants' acceptance of responsibility within their personal relationships. The responsibility felt to fulfill relationship roles served to also be a driver for sexual behavior and acknowledgement of sexual health. The presumed role taken in participants' intimate or sexual relationships, 
in some cases, dictated responsibility to engage in sexual activity or participate in specific behaviors. Participants who were married, described often feeling compelled to engage in sexual activity with their mates as a "duty." "I can say that, in some instances --- and this was when I was married ...--- it was just to do my part as I felt like as, a wife and just go on and give it to him. I don't want it but just go ahead and do it. That was a stressful thing on me, but in a sense, it was like, it was my duty."

Additionally, some of these women's sense of responsibility caused them to engage in sexual activity to prevent infidelity in their intimate relationships. One participant described this by stating: "If it's been months --- if you don't give it to him, he's going out there and get it, so... (If you don't she will - in background) yeah, you'll do it."

These findings indicate that these women's ability to make decisions may have an impact on their view of sexual health and its importance to their overall well-being. Moreover, these findings also reveal that these women's adjustment to achieve overall well-being, their independence, and dedication to intimate relationships and relationship roles have some influence on sexual health and behaviors.

\section{Securing an Attachment}

Securing an attachment is a theme represented by feeling connected to or working to preserve a connection with an individual, group, or religion. Securing an attachment was often proposed by focus group participants as justification for their acknowledgement of sexual health or engagement in various sexual behaviors. The four categories contributing to this theme include: religiosity/spirituality, relational, emotional, and sexual. Religiosity/spirituality was defined for QUAL study purposes as factors relating to or demonstrating one's belief or devotion to God, a higher being, or one's spirit or soul as an entity separate from their physical body. Relational from this perspective continues to focus on those instinctive factors developed by or contributing to the connection between an individual and another person. Emotional has been defined in this study as intuitive factors developed by and/or displayed through a person's feelings. Lastly, sexual focuses on instinctive factors developed by or contributing to one's sexuality and/or the physical connection between an individual and their intimate partner. The compilation of these categories each contributed to the attachment obtained between participants and their intimate or sexual partners.

Both categories relational and sexual were depicted in participants' description of the connection felt between themselves and their partner(s). The connection between these women and their partner(s) was found to be an important part of sustaining a relationship, which was often found to be strengthened through sexual activity. One participant described behaviors extending beyond sexual intercourse that helped to secure an attachment to her mate, by stating: "...more than just the actual sexual act but, you know, feeling connected to one another. You know all the good things that come from feeling close to somebody, like just simple hugs, like eye contact or good conversation, 
and things like that can be perceived as something sexual more than just actually like laying in the bed having sex with somebody."

The category emotional was expressed in participants' description of their own or their partner's feelings. Participants' personal feelings or their partner's feelings were found to foster both the physical and emotional connections felt with their sexual or intimate mate(s). Many of these women described having to secure an emotional or "intimate" connection in their relationships prior to engaging in any sexual activity. Whereas others described engaging in sexual activity in an effort to procure an emotional or "intimate" attachment to another. "...being in a sexual relationship, a healthy sexual relationship. It makes a difference and it brings something to --- well, it makes you appreciate that person and you appreciate yourself for sharing yourself with this person, because they're special to you and you want to share your body with them, and this is something, a closeness that you feel with them...." "I do want to be able to communicate with you. I do want to be able to connect with you on another level other than us having sex, like talking, having a glass of wine and... going to the movies, interacting with family. To me, all of that is intimacy." "To me, intimacy is not sex. [clears throat]... intimacy --- that's what we like ... but we think if we have sex, that's going to keep that coming, but not necessarily. So, what happens is, you kinda get into that one moment, but then if you're with a mate and you're not in a relationship and the person leaves, then you're calling the next Saturday like, 'where are you?'”

On the contrary, failing to secure an attachment was described by focus group participants as a barrier or obstacle that may hinder the physical connection between an individual and their sexual or intimate partner(s). Feelings ensued from engaging in sexual activity without having an attachment to a sexual partner were described by one participant as, "... it feels like ugh, and you get this disgust and this weird feeling about your body."

Ongoing feelings of sadness or symptoms of depression, which was additionally categorized as emotional, also served to prevent securement of an attachment within sexual or intimate relationships. These women's descriptions of the influence of depressive symptoms on sexuality include: "Like if you don't feel like getting out of the bed at all. Why would I want someone else to get into bed?" "I have no desire. I have no desire to even want to have it.... My desire for all of that, is just gone. I just really don't know how to pick it back up. I really do, I want somebody. “

Religiosity/spirituality was depicted by these women's attachment or devotion to God. Participants' religious connections frequently appeared to shape or validate their sexual behavior. Focus group participants discussed how living in the "Bible belt" influenced their awareness of sexual health and expression of their sexuality. One participant described this by saying: “... since we are in the Bible belt...you're not really supposed to be taught about certain things, you're not supposed to know certain things. So, when you become old enough to engage in those sexual practices and you don't know because maybe you know, your family skirted around the issue... you don't really know how to be safe. Or, sexually healthy." 
Additionally, these women's attachment to God could also serve as a source of contention between their religious convictions and sexual behavior. However, this attachment did not appear to have a significant influence on participation in sexual activity or acknowledgement of sexual health. "So, I feel convicted sometimes when I do engage in sex because of that particular saying in the Bible of "not having sex, you know, before marriage." Although I think about it and I'm like 'Oh, Lord forgive me', but then still do it anyway. "“

These findings propose that these women perceive some emotional factors as fundamental to the dynamic of their personal relationships. This suggests a possible relationship between specific emotional, relational, and sexual factors and sexual health or health behavior.

\section{Resolving Ambiguity}

The final theme revealed from the study's QUAL data was resolving ambiguity. Resolving ambiguity expressed participants' uncertainty surrounding many of the aspects in which they assumed responsibility in acquiring a desired level of sexual health. Like the theme "taking responsibility", this theme arises from the same categories which include: assimilation, relational, and self-sufficiency. From the perspective of eliminating uncertainty, this combination of categories each represented areas in which these women identified not having adequate information to permit achieving or maintain their desired level of sexual health. Focus group participants described ambiguity in their familiarization with the current sexual culture, their relationship statuses, and ability to sustain their independence.

The category relational was often depicted in these women's determination of their relationship status. One participant described feelings of confusion in her relationship by stating: "I think I'm in a, I guess, it's a relationship, because, as a- for me sometimes if I'm engaging, I think it's a relationship...."

Assimilation was expressed by many of these women in their description of clarifying measures taken toward resolution or progression toward competence in the achievement of an optimal level of sexual health by finding some resolve. "But I had to get to that point, I had to re-evaluate what was going on, re-evaluate myself, re-evaluate my thinking to realize...." "And, you're thinking, I'm too scared to ask, for a lack of a better word 'Do we go together?' And I discovered very quickly, we didn't go together. Now, going through that ... and learning myself ... that helped me to realize that was not a healthy relationship."

Self-sufficiency was depicted by these women in their efforts to maintain their independence. Resolving ambiguity for some of these women helped them to gain a sense of independence. During focus group interviews, these women discussed the ease of "losing yourself" in sexual or intimate relationships. Many described differences in their views on sexual health after resolving personal issues of uncertainty. One 
participant illustrated this by stating: "I can relate to it and I can feel it and I can hear things and I can see things. I can see things clearer now because I was out of that --- that fog, that negativity, that stress."

As a result of resolving ambiguity, these women illustrated an ability to gain independence and take responsibility for their sexual health and behavior. These women determined that decisions must be made in order to establish personal ideals that were necessary to achieve an optimal state of sexual health. One participant stated, "You have to have standards, you have to put rules in place..." Another indicated: "It all comes down to what you require before you become sexually — engaged with someone."

Similar to those findings obtained from the theme "taking responsibility", these findings suggest that these women's ability to make decisions regarding areas of ambiguity related to their sexuality may be related to their acknowledgement of sexual health and participation in various sexual behaviors. The women in this study may need to resolve issues regarding sexual culture or climate, relationships, and the ability to maintain their independence to achieve desired levels of sexual health.

\section{Integration of Qualitative and Quantitative Data}

Integrating both sets of data involved carrying out an independent interpretation of QUAL and QUAN findings. After completing both analyses, results were defined, summarized, and illustrated using tables to display individual conclusions. Following thematic and regression analyses, comparisons and contrasts between findings were made to identify common topics or subject matter. Findings identified through comparison of QUAL and QUAN analyses were aligned by association across paradigms and displayed in a cross-tabulation table to complete data merging. (Creswell \& Plano Clark, 2011; Lorvick et al., 2012). Initially, QUAN variables examined in this study were compared and merged with categories that comprised the study's final QUAL themes (Table 4-11). This integration reveals that all QUAN variables are not reflected in each QUAL theme generated from these women's perception of sexual health. Additional integration occurred through relationships found to be significant from QUAN analysis and were merged with related themes and categories that emerged from QUAL analysis

(Table 4-12).

\section{The New Normal}

These women's perception of "the new normal" as having an important influence on acquiring or maintaining a desired level of sexual health was recognized thematically as a fundamental component of identified relationships among depressive symptomology and gender inequality. A category materialized from the coding process during QUAL analysis and found to be an important factor contributing to sexual health was 
Table 4-11. Integration of Quantitative Variables with Qualitative Themes.

\begin{tabular}{|c|c|c|c|c|c|}
\hline \multirow[b]{2}{*}{ QUAN Variables } & \multicolumn{5}{|c|}{ QUAL Themes } \\
\hline & "The New Normal" & $\begin{array}{l}\text { Having to } \\
\text { Compromise }\end{array}$ & $\begin{array}{c}\text { Taking } \\
\text { Responsibility }\end{array}$ & $\begin{array}{l}\text { Securing an } \\
\text { Attachment }\end{array}$ & $\begin{array}{l}\text { Resolving } \\
\text { Ambiguity }\end{array}$ \\
\hline Sexual health & - Assimilation & $\begin{array}{ll}\text { - } & \text { Multidimensional } \\
\text { - } & \text { Self-sufficiency } \\
\text { - } & \text { Sexual }\end{array}$ & $\begin{array}{ll}\text { - } & \text { Assimilation } \\
\text { - } & \text { Self- } \\
\text { sufficiency }\end{array}$ & - Sexual & $\begin{array}{ll}\text { - } & \text { Assimilation } \\
\text { - } & \text { Self- } \\
& \text { sufficiency }\end{array}$ \\
\hline $\begin{array}{c}\text { Sexual risk } \\
\text { behavior }\end{array}$ & - Expression & - Sexual & & - Sexual & \\
\hline $\begin{array}{l}\text { Depressive } \\
\text { symptomology }\end{array}$ & & - Self-sufficiency & $\begin{array}{l}\text { - Self- } \\
\text { sufficiency }\end{array}$ & - Emotional & $\begin{array}{ll}\text { - } & \text { Self- } \\
\text { sufficiency }\end{array}$ \\
\hline $\begin{array}{l}\text { Religiosity/ } \\
\text { spirituality }\end{array}$ & - Influence & & - Relational & $\begin{array}{l}\text { - Religiosity/ } \\
\text { spirituality }\end{array}$ & \\
\hline Gender inequality & - Influence & - Relational & - Relational & $\begin{array}{ll}\text { - } & \text { Emotional } \\
\text { - } & \text { Relational }\end{array}$ & - Relational \\
\hline
\end{tabular}

Note. Quantitative (QUAN) variables measured by instruments: Sexual health, sexual risk behavior, depressive symptomology, gender inequality. Sexual health = SQOL-F (Sexual Quality of Life Questionnaire - Female total score); Sexual risk behavior $=$ SAAQ (Sexual Activities and Attitudes Questionnaire sexual activities subscale score); Depressive symptomology $=\mathrm{CESD}-\mathrm{R}(\mathrm{Center}$ for Epidemiologic Studies Depression Scale - Revised total score); Gender inequality = SRPS (Sexual Relationship Power Scale Decision-Making Dominance subscale score). Qualitative (QUAL) themes were derived from a process of coding where data were clustered into categories, and categories were merged into final themes. 
Table 4-12. Integration of Quantitative Inferences with Qualitative Themes.

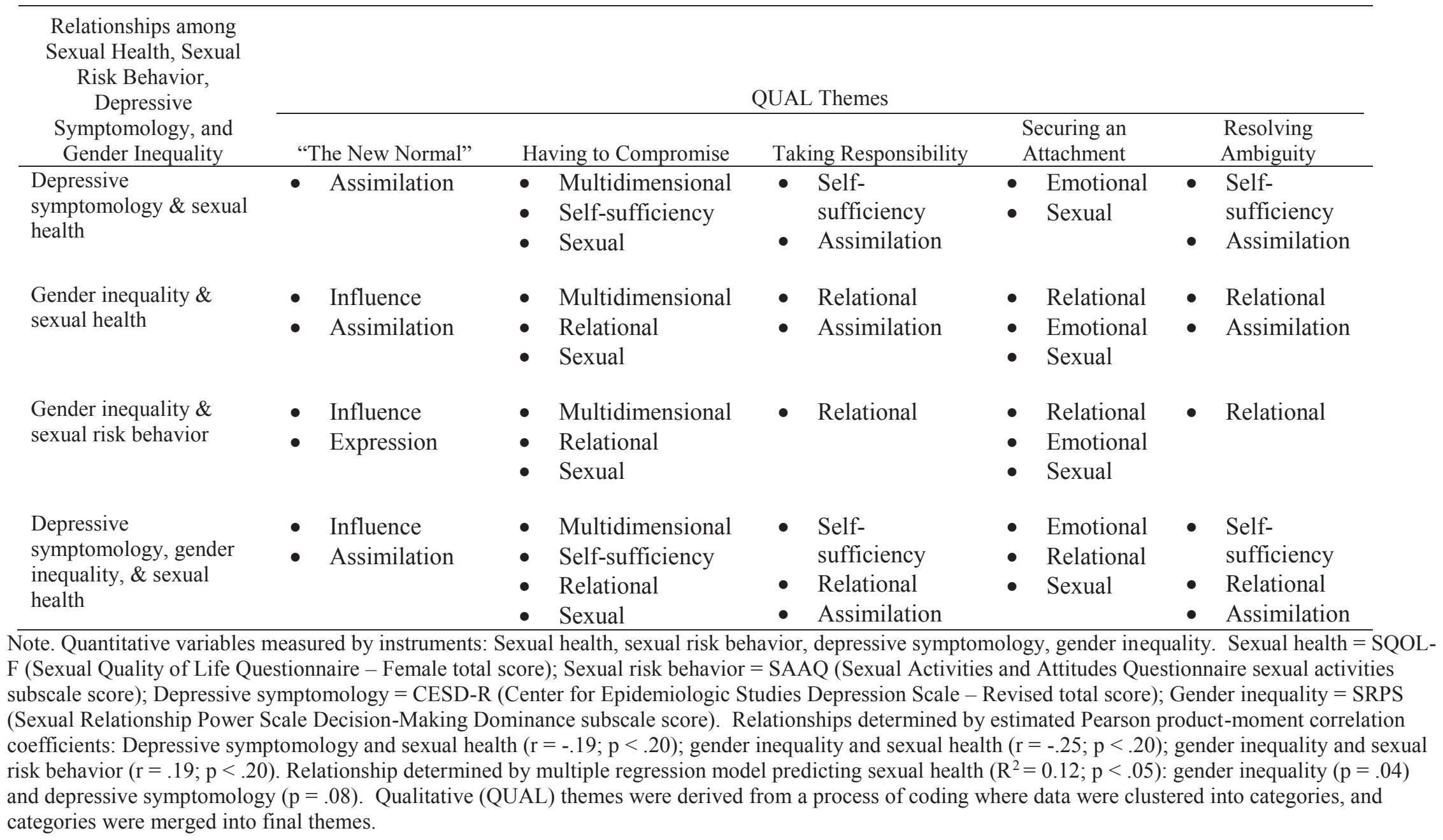


assimilation. Assimilation was a category found to have a consistent presence in QUAN relationships that included sexual health as an outcome variable.

Other categories affiliated with "the new normal" and found to correspond with QUAN findings include: influence and expression. These women discussed the influence of power within their sexual or intimate relationships as a factor involved in their sexual health and behavior. Influence was a category present in QUAN relationships that examined gender inequality. Additionally, "expression", corresponded thematically with the QUAN variable sexual risk behavior.

These women's discussion of sexual health identified an adaptation to the current sexual culture that was influenced by multiple factors. Factors acknowledged by these women as contributors to the adjustment toward sexual well-being included having depressive symptoms and the ability to make decisions within their sexual or intimate relationships. The merging of categories corresponding to "the new normal" with relationships revealed through QUAN analysis serve to augment findings generated from only one perspective. QUAN findings that identified statistical significance among sexual health, depressive symptomology and gender inequality were supported by the thematic affiliation of both assimilation and influence. Additionally, the confirmed relationship between gender inequality and sexual risk behavior was also illuminated through the thematic affiliation of influence and expression.

\section{Having to Compromise}

During focus group interviews, these women acknowledged having to compromise or achieve balance between physical, emotional, and sexual components to maintain a desired level of overall well-being. The negotiation between various aspects of sexual health was recognized as an essential theme represented in all inferred relationships among sexual health, sexual risk behavior, depressive symptomology, and gender inequality. Through the process of QUAL analysis, this negotiation was categorized as "multidimensional". This category was determined to have a clear presence in QUAN relationships that included both sexual health and sexual risk behavior as outcome variables.

Another category identified as having a prominent presence in the over-arching theme of having to compromise is "sexual". In these women's discussion of compromises toward achieving sexual well-being, many described concessions made that were incongruent with their sexual desires. As a result, this category also has a consistent presence in QUAN relationships that included both sexual health and sexual risk behavior as outcome variables.

Other categories affiliated with "having to compromise" and found to correspond with QUAN findings include: self-sufficiency and relational. In these women's discussions, compromise was described when addressing their independence and intimate or sexual relationships. Additionally, these women recognized the presence of depressive 
symptoms as a factor that precluded the ability to maintain their independence and reliance on self for personal sustainability, thus making self-sufficiency a category present in QUAN relationships that examined depressive symptomology. Relational factors corresponded thematically with gender inequality. Compromise related to the category "relational" for focus group participants was directed toward negotiating the "balance" of power within relationships based upon their assumed roles.

The merging of QUAL categories associated with the theme "having to compromise" delves further into relationships revealed through QUAN deduction. The thematic affiliations of multidimensional, self-sufficiency, relational and sexual to identified relationships among sexual health, depressive symptomology and gender inequality were supported QUAN findings determined by statistical significance. Also, the identified connection between the categories: multidimensional, relational, and sexual to the relationship between gender inequality and sexual risk behavior also served to support significant QUAN findings.

\section{Taking Responsibility}

The merging of the QUAL categories assimilation, self-sufficiency, and relational served to explicate relationships corroborated through QUAN analysis. These women perceived the ability to make decisions as imperative to their assumption of responsibility for sexual health. This responsibility was also acknowledged thematically as a factor contributing to their overall level of sexual health. These women's description of responsibility reflected upon the adjustment deemed necessary to assimilate to their sexual culture and/or well-being. Responsibility in adjustment to sexual culture involved educating themselves and engaging in protective behaviors such as using condoms or other methods of contraception. In this case, assimilation is a category derived from QUAL analysis that also signifies "taking responsibility" and maintains a consistent presence in QUAN relationships that include sexual health as an outcome variable.

Other categories affiliated with "taking responsibility" stem from these women's acknowledgement of obligation in their accountability for sexual well-being. These women described being accountable to themselves in the maintenance of their sexual health. Similar to integration related to the theme "having to compromise", depressive symptomology was acknowledged by these women as a factor that complicated the personal sustainability or self-sufficiency required to take adequate responsibility for their sexual health. Accordingly, from this perspective, self-sufficiency corresponds with QUAN relationships that examine depressive symptomology.

In addition, these women recognized a responsibility to their intimate or sexual partner(s) to maintain their sexual health. Some described participation in various behaviors such as, regular STI testing or engaging in sexual activity, as their "duty" to fulfill the role of a wife and/or partner or in effort to maintain an amenable dynamic within their relationships. Thematically, the responsibility these women felt to their 
mates was categorized as "relational". Relative to "taking responsibility" the association of "relational" corresponds to QUAN relationships that examine gender inequality.

While examining the theme "taking responsibility", interpretation of QUAL and QUAN analysis confirm that the affiliation of categories including: assimilation, selfsufficiency, and relational are consistent with QUAN results and validate relationships among sexual health, depressive symptomology and gender inequality. Additionally, this evaluation reveals that thematic affiliation of the category "relational" solely corresponds to the relationship between gender inequality and sexual risk behavior.

\section{Securing an Attachment}

Another concept heavily discussed by these women in the acknowledgment of their sexual health was the desire to "feel close to someone". These women often described the development of personal or intimate connections from either an emotional or sexual standpoint. The integration of QUAL and QUAN data serve to corroborate these findings through thematic associations of the categories: emotional, sexual, and relational.

Similar to interpretation for the theme, "having to compromise", the category "sexual" appears to have a prominent presence in the evaluation of "securing an attachment". For many of these women, feeling connected or working to maintain a connection with their intimate or sexual partners involved physically engaging in sexual activity. This connection also contributed to their own sexuality and the way in which they expressed it. For these reasons, "sexual" was identified as having a consistent presence in QUAN relationships that included both sexual health and sexual risk behavior as outcome variables while acknowledging connections made to help these women achieve a desired level of sexual health.

Other categories associated with "securing an attachment" are related to these women's feelings and the impact of those feelings on the connection between sexual or intimate partners. These women often discussed the importance of establishing an emotional or "mental" connection with their mates which could be influenced by the power present within their relationships. For example, participants who considered themselves to be the dominant figure within their relationships described their mates' difficulty accepting inferiority which often resulted in infrequent sexual activity. Additionally, these women's emotional state, which for some included the presence of depressive symptoms, was also recognized as having an impact on their sexual desire or relationship status. Defined in this study as intuitive factors developed by and/or displayed through a person's feelings, the category "emotional" was recognized as having a meaningful presence in QUAN relationships that examined both depressive symptomology and gender inequality.

Lastly, in the examination of securing an attachment, the category "relational" addressed factors associated with the connection between two individuals. For these 
women, the dynamics present within their sexual or intimate relationships contributed substantially to their sexual well-being. From this perspective, this category generated through QUAL analysis also corresponds to QUAN relationships that examine gender inequality. Subsequently, the integration of the categories: emotional, sexual, and relational validate the significance found within relationships among sexual health, sexual risk behavior, depressive symptomology, and gender inequality.

\section{Resolving Ambiguity}

Integration of QUAL and QUAN analyses also demonstrated a corroboration of findings revealed while investigating the study's final QUAL theme, "resolving ambiguity". During focus group interviews, these women described uncertainty in their adjustment to the existing sexual culture, with their ability to maintain independence, and within their relationships. These factors were often found to interfere with acquiring desired levels of sexual health or well-being for these women. Thus, the merging of data from this perspective revealed thematic affiliation with the categories: assimilation, selfsufficiency, and relational.

Similar to the integration of data conducted for previously mentioned QUAL themes, each QUAN variable examined in relation to the theme "resolving ambiguity" was found to correspond to a relationship determined from QUAL analysis. These women's acknowledgement of doubt or ambiguity in adjusting to the current sexual climate corresponded thematically and demonstrated presence in QUAN relationships that examined the outcome variable, sexual health. In addition, these women's accounts of uncertainty in their ability to maintain independence, a concern which was acknowledged to be complicated by the presence of depressive symptoms, was categorized as "self-sufficiency". This category was found to correspond thematically to QUAN relationships that examined depressive symptomology. Finally, these women's recognition of uncertainty in their sexual or intimate relationships was categorized as "relational". From the standpoint of "resolving ambiguity" the thematic affiliation of "relational" was determined also to have a consistent presence in QUAN relationships that examined gender inequality.

Integration of both analyses support merging the QUAL categories: assimilation, self-sufficiency, and relational to support significant QUAN relationships among depressive symptomology, gender inequality, and sexual health. Further, the identified relationship between gender inequality and sexual risk behavior may be explained exclusively through the thematic association of the QUAL category "relational".

\section{Discussion}

Generally, studies centered on the sexual health of AAW tend to limit their focus on STI and pregnancy prevention. Strategies often associated with improving or maintaining the sexual health of these women concentrate on the promotion of 
abstinence, condom use, and STI screening (Alexander et al., 2014; Centers for Disease Control and Prevention, 2014f). The promotion of such behaviors in AAW has been addressed through several evidence-based, public health initiatives (Centers for Disease Control and Prevention, 2014a; Jemmott, Jemmott, \& O'Leary, 2007; Wingood et al., 2004). While recent studies have begun to expand beyond clear-cut influences on increased sexual risk for AAW, most have not broadly addressed the multiple factors known to contribute to their overall sexual health. Current studies have identified the importance of depressive symptomology or mental illness (Lennon et al., 2012; Mincey \& Norris, 2014; Seth et al., 2011), gender inequality or other issues related to gender roles (Hall \& Pichon, 2014; Nehl et al., 2015), and affiliation with religion or presence in the African American church (Stewart, 2014; T. Williams et al., 2016) in sexual risk reduction for African Americans.

This study collectively addresses factors significant in achieving an optimal state of emotional and relational well-being related to sexuality (e.g., sexual health) for this sample of primarily healthy, educated, middle-aged adult women. This sample, which largely includes recent recipients of reproductive healthcare, acknowledged participating in behaviors that may increase their risk of acquiring STIs or experiencing unintended pregnancy. For example, nearly half of these women reported not using a pregnancy prevention method or contraceptive device. Consistent with research conducted by Biggs, Karasek, and Foster (2012), many women who desired avoiding pregnancy reported engaging in unprotected sex for a variety of reasons. More specifically in this study, women's personal accounts acknowledged participation in risky sexual behavior that was motivated by their intuitive feelings or the existing dynamic within their intimate relationships. The intrinsic factors involved with women's experience of risky sexual behavior are often not considered in the assessment or interventions carried out to target sexual health promotion or risk reduction. By using a theoretical model that focuses exclusively on health and sexual behavior, insights have been gained regarding how emotional and relational factors may influence sexual risks for these women. In doing so, this study proposes using a middle range theory derived from the Sexual Health Model to begin holistically meeting the health needs of AAW (Robinson et al., 2002). This middle range theory suggests that both emotional and relational factors impact the overall wellbeing health of AAW. More specifically, the presence of depressive symptoms and decision-making dominance collectively influence the sexual health of AAW (i.e., the power to make decisions).

Obtained from a perspective grounded in well-being related to sexuality, the purpose of this study was to better understand AAW's sexual health and risk behavior. Women in this study readily recognized the multi-faceted approach required to adequately address their sexual health. However, this approach was often identified as a source of discord between meeting their physical, "mental", relational, and sexual needs. Despite ongoing resolution, these women reported having good overall sexual health. This outcome is one not typically reported in the literature, as focus has often been placed narrowly on outcomes (i.e. STIs, unintended pregnancy) or specific risk behaviors (i.e. condom use, drug use, concurrent partnerships or having multiple sexual partners). However, similar to those in this study, AAW have been found to evaluate their 
sexuality, including their sexual attractiveness, and self-esteem positively (Bancroft et al., 2011). The women in this study report having good overall sexual health with SQOL-F scores averaging slightly above 90 . The use of mixed methods in this study allowed researchers to delve further into these women's characterization of their sexual health and exposed certain factors contributing to their risk. This study's findings reveal that while these women were generally satisfied with their sexual health, everyday struggles with maintaining work-life balance, preserving healthy relationships, exercising selfdetermination, and adjustment to the current sexual culture made maintenance of sexual health difficult.

Existing literature reveals that depressive symptoms are in fact related to participation in sexual risk behavior (Lennon et al., 2012). The women in the current study reported participating in less risky behavior, with average SAAQ scores slightly above 10, perhaps because most had few depressive symptoms. While study results may be inconsistent with previous research focusing on risk behavior and depressive symptomology, the current study's investigation of the broader concept of sexual health is aligned with results found in existing literature. For young AAW, higher levels of depressive symptoms were found to be associated with apprehension in discussing condom use with sexual partners (Seth et al., 2011). Similar associations are reflected in the current study's findings where these women reported having lower levels of depressive symptoms (CESD-R mean score $=8.4$ ), which were significantly correlated with better sexual health. Additionally, the model predicting sexual health for AAW included lower depressive symptoms and decision-making dominance (SPRS mean score $=17$ ). These results are likely to stem from these women suppressing their ability to express sexual confidence, or feelings of reliance related to sexuality, which is encompassed in the wide-ranging concept of sexual health. These findings were corroborated by the study's QUAL results that illustrated these women's perception that the presence of depressive symptoms served as a barrier to maintaining intimate relationships and achieving an optimal level of sexual well-being.

Despite meeting the assumptions for normality, close examination of these women's depressive symptoms revealed a blended distribution of two different groups of women who reacted differently to the presence or absence of these symptoms. While most of these women experienced little to no symptoms of depression, there were three women who experienced symptoms classified as having the probability or actually meeting the criteria for a major depressive episode (Eaton et al., 2004). After removing identified outliers, mean CESD-R scores reported for the study's subsample decreased, indicating the presence of fewer depressive symptoms. With this study having a blended distribution of women experiencing depressive symptoms, a subgroup analysis revealed that the extent to which depressive symptomology was associated with gender inequality, sexual health, and sexual risk behavior depended on whether the outliers were included or not. Associations between depressive symptomology and both sexual health $(\mathrm{r}=-.24 ; \mathrm{p}$ $<.20)$ and sexual risk behavior $(r=.20 ; \mathrm{p}<.20)$ were strengthened after the removal of women with higher depressive symptoms. Conversely, focus group participants who experienced higher levels of depressive symptoms did not describe engaging in increased risky behavior. Rather, these women expressed a marked decrease in engagement of 
sexual activity, which, in turn prohibited them from maintaining their desired level of sexual health. This study's QUAN and QUAL findings for both samples support the evaluation of depressive symptoms as a comprehensive measure to address well-being for AAW.

Additional findings from this study reveal that decision-making dominance, a subscale used to measure gender inequality, is negatively associated with sexual health. Notably, the women in this study reported having high levels of power within their intimate or sexual relationships with comparably high levels of decision-making dominance. Typically, SPRS total scores are reported when evaluating gender inequality in AAW, thus making accurate comparisons in this study difficult. However, in previous studies, middle aged AAW report having lower relationship and decision-making power (Altschuler \& Rhee, 2015), unlike the women in the current study. Decreased levels of decision-making dominance were determined to predict improvement women's sexual health, despite these women perceiving their decision-making dominance as being high. This could be explained to some degree through QUAL study findings that revealed these women's innate desire to "secure an attachment" with their partners. These women often described making decisions or participating in behaviors in an effort to establish or maintain an attachment with their sexual or intimate partner(s). Additionally, these women also discussed "having to compromise" to acknowledge their partner's feelings or to fulfill expected relationship roles. This may indicate that these women consciously made decisions to be less dominant within their intimate relationships to foster a neutral power dynamic within their relationships.

This study's findings related to decision-making dominance and sexual risk behavior are consistent with existing literature. Previous studies of heterosexual relationships among AAW assert that those with increased power within their relationships are more likely to have an increased ability to make sexual decisions, such as using condoms or engaging in other protective behaviors (Harvey \& Bird, 2004; Harvey, Bird, Galavotti, Duncan, \& Greenberg, 2002). On the other hand, we found that for the women in this study, having the ability to make decisions within their relationships, as indicated from SRPS scores, did not consistently translate to making sexually healthy decisions.

As previously mentioned, nearly half of the women participating in this study reported not using a pregnancy prevention method or contraceptive device. This may be explained to some degree by the study's QUAL findings that divulged these women's inherent desire to secure an emotional connection with their partners. During focus group interviews these women often described making decisions that were motivated by both their own and their partner's feelings in effort to either procure or preserve their attachment to another individual. Recent studies have found that AAW's participation in protective behaviors may in fact be mediated by the composition of their sexual relationships (Nehl et al., 2015; Zembe, Townsend, Thorson, Silberschmidt, \& Ekstrom, 2015). To that fact, AAW in long-term relationships or those who are frequently involved with a "main" causal partner find it more difficult to make decisions regarding condom use (Noar et al., 2012). Furthermore, this study’s QUAL results reveal that the 
assumption of roles within their relationships also served to influence their sexual behavior. To fulfill the role of a wife or significant other, these women often described engaging in sexual behaviors or a variety of activities with which they were "initially" uncomfortable, such as having unprotected sex. These women's desire to meet their emotional and relational needs may supersede their extrinsic ability to make decisions that are sexually healthy. In addition to inequities in power, this study's results indicate that relational factors including the composition of sexual relationships, sex partner types, or other issues related to gender roles in relationships contribute to AAW's sexual health and risk behavior.

Finally, this study's QUAN results indicate that sexual health may be predicted by decision-making dominance and depressive symptomology. While the presence of depressive symptoms does not independently influence sexual health, decision making dominance does exhibit predictive properties when considering these women's overall sexual well-being. These results support existing research which posits that the presence of depressive symptoms are likely to prohibit sexual decision making and influence risky sexual behavior (Seth et al., 2011). Likewise, the relationship between both variables and sexual health were corroborated by personal accounts revealing interference in these women's ability to make their sexual health a priority, resolve areas of uncertainty and maintain independence in their intimate relationships while experiencing depressive symptoms. Thus, the integration of QUAN and QUAL data further explicate individual analyses, both of which expose this relationship. When looking specifically at the QUAL theme "having to compromise", a theme which reflected these women's ability to negotiate or make choices, the emergence of categories affiliated with this relationship include: multidimensional, self-sufficiency, relational and sexual. These findings suggest that a host of factors are involved in AAW's sexual health and participation in behaviors that increase their sexual risk. In this study, women's sexual health, while perceived to be "good", was adversely influenced by emotional issues linked to mental health and those that demonstrated feelings of intimacy and attachment. Additionally, relational issues that characterized the interpersonal dynamic between these women and their intimate or sexual partner(s) affected women's decisions to engage in protective behaviors such as condom or other contraceptive use.

Results from the current study show that religiosity and spirituality were insignificant factors correlated to the sexual health and risk behavior of these women. Historically, African Americans have been the racial or ethnic group most likely to believe in God, be affiliated with a church or organized religion, and be devout in their religious practices (Pew Research Center, 2015; Udell, Donenberg, \& Emerson, 2011; Wingood et al., 2013). These results correspond to the current study's QUAN findings obtained from the Values and Beliefs subscale of the BMMRS, which indicate these women's increased orientation to engage in behaviors that are encouraged by their faith. Previous research indicates that religiosity has demonstrated a positive influence on younger AAW's sexual health outcomes (McCree, Wingood, DiClemente, Davies, \& Harrington, 2003). However, in the current study, relationships between spirituality/religiosity and both of the study's outcome variables, sexual health and sexual risk behavior, were not statistically significant. These findings may stem from the lack of 
variability in scores used to quantify spirituality/religiosity in these women. This study's QUAL findings illuminate QUAN results in these women's depiction of their dedication to God and religious beliefs impacted their sexual health and behavior. During focus group interviews, these women's accounts of their lifestyles and personal standards included quotations from biblical scripture, references made to heaven and hell, and reflected their participation in religious activities such as praying and attending church. Despite these women's obvious stated devotion to God, these women described engaging in behaviors that may not have been congruent with their religious beliefs. Currently, both research and clinical practice have begun to explore the role of faith-based organizations in STI and HIV prevention for AAW (Stewart, 2014; T. Williams et al., 2014; Wingood et al., 2013). While efforts to use churches or faith-based measures in sexual health prevention for AAW continue in their development, future research may need to consider religious options only as one of the many available alternatives in the implementation of interventions aimed toward improving the behavior of AAW. Additionally, opportunities for introspection on the part of faith-based organizations should be given to determine suitable approaches to be taken by churches and similar organizations toward sexual health promotion.

Use of a mixed methods design in this study authenticates important findings by adding depth and clarity to issues surrounding the sexual health and behaviors of AAW. Current literature has reflected increases in the perspective that sexual health and health outcomes related to sexual behavior involve a myriad of complex, all-inclusive factors (Douglas \& Fenton, 2013; Satcher, 2013; Sharpe et al., 2012). In this case, the exploration of such phenomena extends beyond the scale of using a single research methodology (Morse \& Niehaus, 2009). This study's QUAN component revealed important associations between sexual health and specific emotional and relational factors. The study's QUAL component complemented these results by incorporating AAW's perception of how the circumstances of their lives influenced their risk and overall sexual health. The integration of both methods provided a perspective that could not have been captured using quantitative or qualitative measures alone.

\section{Limitations and Strengths}

Several limitations have been identified for this study. First, the study's sample, while appropriate in size to yield significant results from the study's QUAL component, may have been slightly underpowered to adequately detect significant relationships from the study's QUAN component. QUAN results revealing trends toward significance may be attributed to the study's relatively small sample size. Associations between religiosity/spirituality and both outcome variables and the influence of decision-making dominance on sexual risk behavior may have been difficult to determine based on the number of units required to determine statistical significance. A retrospectively conducted power analysis revealed that a sample size of 264 women will be required to achieve a power of .70 with a significance of alpha $=0.05$ in this study. Future studies carried out to examine these or similar phenomena will need to include much larger samples of diverse AAW. 
Findings yielded from this study's exploratory design may only be reflective of its unique sample. Thus, making inferences based on this small, homogeneous group of women may make generalizability of both QUAN and QUAL findings difficult. Despite the QUAL sample closely representing the study's QUAN sample, this group of conveniently selected, highly educated women obtained from a large, southeastern, urban community is not sufficient for representing AAW from other areas. The women participating in this study largely reported having ease of accessibility and being recent recipients of sexual and reproductive healthcare, leaving a low representation of women with little or those without access to healthcare at all. Additionally, with the women participating in this study being fairly healthy, the determination of variables influencing the sexual health and risk behaviors of women with chronic comorbid conditions or poor mental health cannot be made from this study.

The credibility of this study's findings relies heavily on the candor of its respondents. With this study investigating topics of a highly sensitive nature, the selfreport of data collected may be subject to respondent bias. Both QUAN and QUAL data required participants' to provide information based on their ability to recall information that occurred in the past. It is possible that the veracity of provided responses did not accurately depict the events, actions, and thoughts of the women participating in this study. It is also possible that QUAN survey data and QUAL data collected during focus group interviews was subject to social desirability bias. Due to the nature of the research topic, these women may have chosen to provide responses that were not entirely transparent or were positively portrayed to seek social approval and eliminate judgement. Being asked to respond to personal questions in a group setting, particularly questions about risky behavior, may have led to underreporting in an effort to provide politically acceptable responses.

Despite its limitations, this study has several strengths. By taking a holistic approach anchored in well-being related to sexuality, this study expands upon existing literature that focuses narrowly on sexual risk reduction for AAW. Using selected emotional and relational factors derived from Robinson et al.'s (2002) Sexual Health Model allows for several factors known to influence AAW's sexual health and risk behavior to be collectively addressed. Placing emphasis on the broader concept of sexual health provides a perspective that acknowledges social determinants of health that specifically impact these AAW (Sharpe et al., 2012). Factors including the surrender of power within women's relationships, being sexually liberated, exposure to sexual networks which comprised "the new normal", and engaging in concurrent sexual partnerships were all recognized as influencing women's sexual health or potentially increasing their risk. In taking this perspective, a glimpse into AAW's behavior has begun to reveal the complexity underlying and contributing to their sexual health. These findings may be used as a complement to traditional approaches taken to promote women's health, reduce risk, and encourage sustainable behavior change in AAW.

Another strength of this study lies in the methodology employed in the study's design. With the concept of sexual health emerging in its role to improve health outcomes for AAW, the exploration of issues that contribute to this state of well-being 
was enhanced by using two modes of inquiry. The combination of quantitative and qualitative research methods in this study provides an innovative perspective that uniquely contributes to the existing body of knowledge related to sexual health. To date, research investigating sexual health in AAW has not used a mixed methods design in its approach. The integration of QUAN and QUAL data revealed that AAW's sexual risk cannot be adequately reduced solely through the use of behavioral interventions that provide education regarding consistent condom use or STI screening. Rather, women's feelings and interpersonal characteristics should be considered to complement existing interventions to include the healthy expression of sexuality, promotion of emotional wellbeing, and the recognition or acceptance of assumed relationship roles.

Furthermore, this study unveils important findings from a largely understudied population. Evidence generated from the conduct of this study was obtained from a sample of well-educated AAW residing within a large, urban community in the southeastern United States. With the southeastern US being a region heavily affected by disproportionate rates of STIs and both teenage and unintended pregnancy, many research studies tend to concentrate on the sexual behaviors of women from this region (Centers for Disease Control and Prevention, 2014c; Guttmacher Institute, 2013; McLellan-Lemal et al., 2012; National Campaign to Prevent Teen and Unplanned Pregnancy, 2016). While most study samples include younger women of lower socioeconomic status (Cates et al., 2015; Painter, Herbst, Diallo, \& White, 2014; Sales, DiClemente, Davis, \& Sullivan, 2012; Wingood et al., 2013), the current study reveals factors contributing to women's risk that may differ based on maturity, life course, or progression through various life stages. The results from this study provide insight into the thoughts and behaviors of middle-aged adult women from this region, who for the most part were formally employed with employer-provided insurance coverage. For many of these women, being aware of how STIs are transmitted, knowing the causes of unintended pregnancy, and/or being aware of their STI/HIV status did not eliminate their participation in risky sexual behavior. Rather, this study's findings indicate that emotional and relational issues surrounding intimacy, marital discord or divorce, the pursuit of family-work-life balance, or loss of libido in the context of depressive symptoms may contribute to middle-aged AAW's risk and negatively influence their overall sexual well-being.

\section{Practice and Policy Implications}

The notion of sexual health as a holistic approach that could be used to improve the negative health outcomes experienced by AAW is relevant to both clinical practice and public health policy. Implications for healthcare providers highlighted from the conduct of this research involve recognizing the importance of addressing each of the domains that contribute to the sexual health of AAW. The concept of sexual health may be used by healthcare providers to empower AAW to make healthy decisions by encouraging the acknowledgement of their sexuality, emotional abilities, and the configuration of their intimate or sexual relationships. Taking measures to not only address the resultant consequences of women's behavior, but to also include pertinent 
emotional, relational, and sexual matters underlying those behaviors could serve to complement current models of care which lack necessary depth in their tactics. Existing interventions that focus on abstinence, consistent condom use, and regular STI screening, while helpful in their intentions fail to specifically address several factors that women grapple with in their daily life experiences. Expanding the scope of current preventive care efforts by integrating the issues identified in this study to include: depressive symptomology and emotional wellness, decision-making dominance, assimilation, and self-sufficiency. All of these factors may help in the development of interventions that promote sustainable behavior change in AAW. With the findings of this study indicating the intricate combination of dimensions involved in women's sexual behavior, healthcare providers should be on the forefront of promoting sexual health as an all-inclusive mode by which the development of successful risk reduction interventions may be derived for AAW. Doing this will require education of clinicians regarding the multiple dimensions involved in sexual health to effectively enlighten AAW regarding the possibilities that this perspective may bring to improve their overall health in the future.

Implications for public policy involve increasing the range of services included in sexual healthcare for women. Currently, preventive services focus largely on the reproductive aspect of women's health. Approved guidelines for insurance coverage for annual well-women visits include age appropriate services that involve: screening for cervical cancer, diabetes and interpersonal violence, testing for human papillomavirus, or counseling for STIs, contraception, and breastfeeding (Office on Women's Health \& US Department of Health and Human Services, 2016; U.S. Department of Health and Human Services Health Resources and Services Administration, 2016). However, findings from this study reveal the need to broaden these services by including those that address the emotional and relational components known to be involved in women's behavior. Despite being exploratory in nature, this study delineates areas of interest for both policymakers and organizations involved in the modification of current legislation designed to improve the health outcomes of those affected by sexual health disparities. For example, creating a payment model that will permit the inclusion of additional time to discuss sexual health matters or services such as routine depression screenings, the completion of mental health or emotional well-being scales, or assessments of the dynamic within women's interpersonal relationships may all improve the disproportionate rate of negative health outcomes affecting AAW.

Through the identification of specific factors contributing to AAWs sexual health, this study provides political insight into the direction in which sexual health research, women's healthcare programs, and the surveillance of women's health outcomes should be headed. Further research will be required to determine the exact factors or interventions essential to include in actual design of federal recommendations or mandated guidelines that encompass the wide-ranging perspective of sexual health. Allocating federal, state, or local funds specifically for the conduct of sexual health research may help to expand this body of knowledge and possibly aid in the identification of causal relationships for AAW and other groups affected by sexual health disparities. 
Additionally, implementing national or state-wide programs and initiatives to promote sexual health that include strategies to educate women not only on STI prevention and reproductive health, but also on the importance and sexual influence of emotional well-being and relationship or interpersonal abilities to achieve an overall state of wellness. This study highlights the importance of expanding current measures used to gauge the multiple dimensions of sexual health (Douglas \& Fenton, 2013). The conduct of multi-site studies will expand sexual health promotion initiatives while delineating specific factors influencing the sexual health of groups throughout the nation.

Furthermore, national databases will need to be designed to adequately examine each of the factors known to contribute to sexual health. This will require moving away from the nation's current of practice of using the inefficient measures of sexual risk behavior and negative health outcomes to evaluate the more comprehensive concept of sexual health.

\section{Theoretical Implications}

As previously mentioned, use of the Sexual Health Model (SHM) in this study provides a perspective that specifically addresses the needs of AAW. In its preliminary form, the Sexual Health Model may be seen as a grand theory composed of several broad concepts. Most notably, the model seeks to comprehensively integrate sexual, relational, and emotional dimensions that contribute to continued sexual risk (Robinson et al., 2002). This study aims to propose a middle range theory by narrowing the model in its original form to include emotional and relational factors known to influence AAWs sexual health and risk behavior. Additionally, this study collectively examines relationships among specified factors that have not previously been tested. While the model tested in this study explains only $12 \%$ of the variance in AAW's sexual health, only two of the ten spokes included in the original SHM were tested in this sample of AAW. Results from this study give merit to the model as tested, while findings indicate the necessity to expand the tested model to integrate other pertinent variables.

The conduct of this mixed methods research study supported several components of the SHM (2002). Specifically, the current study significantly validated inclusion of the "intimacy and relationships" and "challenges" spokes on the wheel used to depict the original SHM (Figure 1-1). This study's QUAN and QUAL findings confirm including gender inequality as a pertinent factor reflecting intimacy and relationships, and depressive symptomology as a challenge to achieving an optimal level of sexual health for AAW. Conversely, QUAL study findings revealing these women's stated devotion to God only marginally support including the "spirituality" spoke on the wheel of sexual health. QUAN study findings related to religiosity/spiritualty corroborate QUAL study results through its nonsignificant associations with sexual health and risk behavior. However, these women uniformly scored very high on the religiosity/spirituality instrument used in this study. In this study, both women's perceptions and statistical analyses were used to substantiate the importance of spokes on the wheel and warrant maintaining specific factors' inclusion or exclusion from the model. 
The theoretical perspective tested in this model was generally confirmed through the conduct of this research study. The women participating in this study overwhelmingly described factors that were both emotional and relational in nature, as having a considerable impact on their sexual health and participation in risky sexual behavior. The combination of issues that derived either from these women's feelings, mental state, and intimate or sexual relationships served to influence many of decisions made related to their sexual health and behavior. These women's depiction of engaging in various behaviors based upon their pursuit of an intimate relationship, established relationship roles, desire to "feel close" to their partners, or to avoid hurting their partners' feelings confirm the inclusion of emotional and relational factors in the adapted Sexual Health Model as appropriate in this study.

In looking at the specific factors being tested in the model, study findings only marginally support the proposed model in its current form. These women's recognition of the importance in making healthy relationship decisions and acknowledgment of the presence of depressive symptoms as a barrier to maintaining their sexual health, support the inclusion of both gender inequality and depressive symptomology in the adapted model. Both the study's QUAN and QUAL analysis verify the presence of relationships among depressive symptomology, decision-making dominance as a subscale of gender inequality, and sexual health. The use of multiple regression models also confirms the inclusion of these variables in future adaptations of the model. Furthermore, the emergence of issues surrounding attachment, assimilation or adjustment, compromise and accountability in the study's QUAL data further support the significance of these variables in the examination of sexual health in these women.

However, the inclusion of religiosity and spirituality was not well supported by the study's findings, despite being well documented in the literature. While these women described having a religious connection to God, this connection did not emerge as essential to decisions made regarding sexual behavior or maintenance of sexual health. Thus, religiosity/spirituality may need to be removed from the proposed model and perhaps replaced with an additional emotional, relational, or sexual factor. However, consideration must be given to the fact that the sample investigated in this study was highly homogenous, whereas other samples of AAW may not resemble the same degree of uniformity. Additional studies conducted within this population are needed to confirm the removal of this variable.

Additional modification of this model is required to accurately determine the factors that most effectively address the holistic concept of sexual health for AAW. The identified complexity of women's sexual health and behavior will make future revisions a challenging and ongoing undertaking. The identification of pertinent variables to be included in the model and their interactions will need to be re-evaluated and may vary for different groups. Another consideration in future modification of the model will be the duality involved in selected variables. For example, these women described factors that influenced decisions to maintain their sexual health or contributed to their risk that concurrently met their personal sexual needs, strengthened the connection between themselves and their mates, and fulfilled their responsibility as a partner within their 
relationships. Limiting the model to specific emotional and relational factors, may negate the diversity and duality recognized in women's experiences. The inclusion of broad concepts serves as both a challenge and advantage of this model. The challenge lies in encompassing depth in the use of such broad concepts, which can have the potential to yield many issues that reflect the experiences of the women being investigated. Conversely, the perspective generated from the model's extensive foundation can both provide insight into the multiple factors involved in improving the health outcomes of individuals, in addition to revealing the factors necessary to tailor interventions to meet the specific needs of those in certain groups.

\section{Recommendations for Future Research}

While this study sheds light on the multiple facets involved in AAWs sexual health and risk behavior, it also raises several suggestions for future research. Key recommendations involved in future sexual health research involves proper modification the Sexual Health Model. The original model as developed by Robinson et al (2002), in its preliminary form will have to be adjusted to meet the needs of those being investigated. Findings from the study reveal that additional work examining the sexual health of AAW should integrate factors into the model that directly reflect women's decision-making, acknowledge their independence and the composition of their relationships in an effort to broadly address factors pertinent to their sexual well-being. Additionally, further work collectively examining multiple factors known to influence women's sexual health will need to include much larger samples. Increasing the sample size of women being examined is important for two reasons. First, larger samples of women that better reflect the makeup of the population of AAW will enhance determinations made regarding relationships between specific variables. Secondly, increases in studies' sample sizes will likely increase the generalizability of studies' findings. A more diverse sample of AAW may be obtained by enlarging the number of study participants being investigated. Lastly, adjusting the study's design to one that is longitudinal can produce results that will better predict women's outcomes over time. Extending future studies' observation period beyond one time point can contribute to the development of interventions that promote sustainable behavior change and decreased sexual health disparity in AAW.

\section{Conclusion}

By taking a holistic approach to better understand AAW's sexual behavior, this study provided important insights into the multiple factors involved in achieving an optimal level of sexual health. This study successfully employed the use of an innovative model that could be used to complement existing theories and strategies used to improve sexual health outcomes for both younger and older AAW. The development of interventions designed to reduce sexual risk and improve sexual health in AAW should include pertinent emotional, relational, and sexual factors involved in women's sexual behavior. Consideration of women's sexual experiences to include, "the new normal", 
the composition of sexual relationships, and ability to make decisions must be recognized by health care providers as integral to AAW's overall well-being. Going forward, taking a broader approach toward sexual health may significantly contribute to the improvement of disparate health outcomes among AAW. 


\section{LIST OF REFERENCES}

Aalsma, MC, Woodrome, SE, Downs, SM, Hensel, DJ, Zimet, GD, Orr, DP, \& Fortenberry, J. (2013). Developmental trajectories of religiosity, sexual conservatism and sexual behavior among female adolescents. Journal of Adolescence, 36(6), 1193-1204. doi: 10.1016/j.adolescence.2013.08.005

Adimora, A, Schoebach, V, Taylor, E, Khan, M , Scwartz, R , \& Miller, W. (2013). Sex ratio, poverty, and concurrent partnerships among men and women in the United States: a multilevel analysis. Annals of Epidemiology, 23(11), 716-719. doi: 10.1016/j.annepidem.2013.08.002

Adimora, A, Schoenbach, V, Taylor, E, Khan, M, \& Schwartz, R. (2011). Concurrent partnerships, nonmonogamous partners, and substance use among women in the United States. American Journal of Public Health, 101(1), 128-136. doi: 10.2105/ajph.2009.174292

Ajzen, Icek. (1991). The theory of planned behavior. Organizational Behavior and Human Decision Processes, 50(2), 179-211.

Alexander, K, Jemmott, L, Teitelman, A, \& D'Antonio, P. (2014). Addressing sexual health behaviour during emerging adulthood: A critical review of the literature. Journal of Clinical Nursing, 24(1-2), 4-18. doi: 10.1111/jocn.12640

Altschuler, J., \& Rhee, S. (2015). Relationship Power, Sexual Decision Making, and HIV Risk Among Midlife and Older Women. Journal of Women \& Aging, 27(4), 290308. doi: 10.1080/08952841.2014.954499

American Sexual Health Association. (2015). Understanding Sexual Health, 2015, from http://www.ashasexualhealth.org/sexual-health/

American Society for Reproductive Medicine. (2016). Sexual Health, 2016, from http://www.reproductivefacts.org/awards/detail.aspx?id=10694

Bancroft, J., Long, J. S., \& McCabe, J. (2011). Sexual well-being: a comparison of U.S. black and white women in heterosexual relationships. Archives of Sexual Behavior, 40(4), 725-740. doi: 10.1007/s10508-010-9679-z

Biello, K. B., Ickovics, J., Niccolai, L., Lin, H., \& Kershaw, T. (2013). Racial differences in age at first sexual intercourse: residential racial segregation and the black-white disparity among U.S. adolescents. Public Health Reports, 128 Suppl 1, 23-32.

Biggs, M. A., Karasek, D., \& Foster, D. G. (2012). Unprotected intercourse among women wanting to avoid pregnancy: attitudes, behaviors, and beliefs. Womens Health Issues, 22(3), e311-318. doi: 10.1016/j.whi.2012.03.003 
Blanc, A. K. (2001). The effect of power in sexual relationships on sexual and reproductive health: an examination of the evidence. Studies in Family Planning, 32(3), 189-213.

Bouchard, S., Godbout, N., \& Sabourin, S. (2009). Sexual attitudes and activities in women with borderline personality disorder involved in romantic relationships. Journal of Sex \& Marital Therapy, 35(2), 106-121. doi: $10.1080 / 00926230802712301$

Brathwaite, A. (2002). Selection of a Conceptual Model/Framework for Guiding Research Interventions. The Internet Journal of Advanced Nursing Practice, 6(1), $1-8$.

Brawner, B. M., Gomes, M. M., Jemmott, L. S., Deatrick, J. A., \& Coleman, C. L. (2012). Clinical depression and HIV risk-related sexual behaviors among AfricanAmerican adolescent females: unmasking the numbers. AIDS Care, 24(5), 618625. doi: 10.1080/09540121.2011.630344

Brown, D. L., Webb-Bradley, T., Cobb, P. D., Spaw, D., \& Aldridge, K. N. (2014). African American women's safer sexual practices: the influence of ethnic-racial socialisation and body esteem. Culture, Health \& Sexuality, 16(5), 518-532. doi: $10.1080 / 13691058.2014 .891048$

Carter, MW, Kraft, JM , Hatfield-Timajchy, K, Snead, MC , Ozeryansky, L, Fasula, AM , . . Kourtis, AP. (2013). The reproductive health behaviors of HIV-infected young women in the United States: A literature review. AIDS Patient Care and STDs, 27(12), 669-680. doi: 10.1089/apc.2013.0208.

Cates, J. R., Francis, D. B., Ramirez, C., Brown, J. D., Schoenbach, V. J., Fortune, T., . . . Adimora, A. A. (2015). Reducing Concurrent Sexual Partnerships Among Blacks in the Rural Southeastern United States: Development of Narrative Messages for a Radio Campaign. Journal of Health Communication, 20(11), 1264-1274. doi: 10.1080/10810730.2015.1018643

Center for Innovative Public Health Research. (2014). CESD-R: Center for Epidemiological Studies Depression Scale Revised Online Depression Assessment Retrieved September 24,, 2014, from http://cesd-r.com

Centers for Disease Control and Prevention. (2013). Health Disparities and Inequalities Report, from http://www.cdc.gov/minorityhealth/CHDIReport.html

Centers for Disease Control and Prevention. (2014a). Health Disparities in HIV/AIDS, Viral Hepatitis, STDs, and TB Retrieved May 28, 2014, from http://www.cdc.gov/nchhstp/healthdisparities/africanamericans.html

Centers for Disease Control and Prevention. (2014b). HIV among Women, from http://www.cdc.gov/hiv/group/gender/women/ 
Centers for Disease Control and Prevention. (2014c). HIV in the United States: At A Glance Retrieved May 28,, 2014, from http://www.cdc.gov/hiv/statistics/basics/ataglance.html

Centers for Disease Control and Prevention. (2014d). Minority Health - Populations, 2014, from http://www.cdc.gov/minorityhealth/populations/REMP/black.html

Centers for Disease Control and Prevention. (2014e). Sexual Health Retrieved May 28, 2014 2014, from http://www.cdc.gov/sexualhealth/default.html

Centers for Disease Control and Prevention. (2014f). STDs in Racial and Ethnic Minorities Retrieved May 28, 2014, from http://www.cdc.gov/std/stats12/minorities.htm

Centers for Disease Control and Prevention \& HRSA Advisory Committee on HIV, Viral Hepatitis and STD Prevention and Treatment. (2012). Draft record of the proceedings; 2012 May 8-9. Retrieved from http://www.cdc.gov/maso/facm/pdfs/CHACHSPT/20120508_CHAC.pdf.

Connell, R. (1987). Gender and Power. Stanford, CA: Stanford University Press.

Creswell, J. W., \& Plano Clark, V.L. (2011). Designing and conducting mixed methods research (2nd ed.). Los Angeles, CA: Sage.

Crosby, R. A., DiClemente, R. J., Salazar, L. F., Wingood, G. M., McDermott-Sales, J., Young, A. M., \& Rose, E. (2013). Predictors of consistent condom use among young African American women. AIDS and Behavior, 17(3), 865-871. doi: 10.1007/s10461-011-9998-7

D’Souza, G, Cullen, K, Bowie, J , Thorpe, R, \& Fakhry, C. (2014). Differences in oral sexual behaviors by gender, age, and race explain observed differences in prevalence of oral human papillomavirus infection. PLoS One, 9(1), e86023. doi: 10.1371/journal.pone.0086023

Damani, R., Ross, M. W., Aral, S. O., Berman, S., St Lawrence, J., \& Williams, M. L. (2009). Emotional intimacy predicts condom use: findings in a group at high sexually transmitted disease risk. International Journal of STD \& AIDS, 20(11), 761-764. doi: 10.1258/ijsa.2009.009238

DePadilla, L., Windle, M., Wingood, G., Cooper, H., \& DiClemente, R. (2011). Condom use among young women: modeling the theory of gender and power. Health Psychology, 30(3), 310-319. doi: 10.1037/a0022871

Department of Health and Human Services (US), Office of the Surgeon General. (2001). The Surgeon General's call to action to promote sexual health and responsible behavior. Washington, HHS Office of the Surgeon General. 
DiClemente, RJ. (2001). Development of programmes for enhancing sexual health. Lancet, 358(9293), 1828-1829. doi: 10.1016/S0140-6736(01)06878-7

Douglas, J. (2011, August 11). Advancing a public health approach to improve sexual health in the United States: A framework for national efforts. Paper presented at the International Perspectives on Sexual Health National HIV Prevention Conference, Atlanta, GA.

Douglas, J, \& Fenton, K. (2013). Understanding sexual health and its role in more effective prevention programs. Public Health Reports, 128(Supp 1), 1 - 4.

Eaton, WW, Muntaner, C, Smith, C, Tien, A , \& Ybarra, M. (2004). Center for Epidemiologic Studies Depression Scale: Review and revision (CESD and CESDR). The Use of Psychological Testing for Treatment Planning and Outcomes Assessment: Instruments for Adults (3rd ed.). Mahwah, NJ: Lawrence Erlbaum.

Fawcett, J. (2005). Contemporary Nursing Knowledge: Analysis and Evaluation of Nursing Models and Theories (2nd ed.). Philadelphia: F.A. Davis Company.

Fetzer Institute, \& National Institute on Aging Working Group. (1999). Multidimensional measurement of religiousness/spirituality for use in health research: A report of the Fetzer Institute/National Institute on Aging Working Group.

Fichtenberg, C. M., Jennings, J. M., Glass, T. A., \& Ellen, J. M. (2010). Neighborhood socioeconomic environment and sexual network position. Journal of Urban Health : Bulletin of the New York Academy of Medicine, 87(2), 225-235. doi: 10.1007/s11524-009-9425-9

Ford, J. V, Barnes, R, Rompalo, A, \& Hook III, E. W. (2013). Sexual health training and education in the US. Public Health Reports, 128(Supp 1), 96-101.

Fugl-Meyer, A. R., Lodnert, G., Branholm, I. B., \& Fugl-Meyer, K. S. (1997). On life satisfaction in male erectile dysfunction. International Journal of Impotence Research, 9(3), 141-148.

Green, J., \& Thorogood, N. (2009). Qualitative Methods for Health Research: SAGE.

Grieb, S. M., Davey-Rothwell, M., \& Latkin, C. A. (2012a). Concurrent sexual partnerships among urban African American high-risk women with main sex partners. AIDS and Behavior, 16(2), 323-333. doi: 10.1007/s10461-011-9954-6

Grieb, S. M., Davey-Rothwell, M., \& Latkin, C. A. (2012b). Social and sexual network characteristics and concurrent sexual partnerships among urban African American high-risk women with main sex partners. AIDS and Behavior, 16(4), 882-889. doi: $10.1007 / \mathrm{s} 10461-011-0030-\mathrm{z}$

Guttmacher Institute. (2013). Unintended Pregnancy in the United States, 2015, from https:/www.guttmacher.org/pubs/FB-Unintended-Pregnancy-US.html 
Hall, N. M., \& Pichon, L. C. (2014). Gender roles, sociosexuality, and sexual behavior among US Black women. Health Psychology and Behavioral Medicine, 2(1), 171-182. doi: 10.1080/21642850.2014.882236

Harris, S, Sherritt, L, Holder, D, Kulig, J, Shrier, L, \& Knight, J. (2008). Reiability and vidity of the Brief Multidimensional Measure of Religiousness/Spirituality among adolescents. Journal of Religion \& Health, 47, 438-457.

Harvey, S. M., \& Bird, S. T. (2004). What makes women feel powerful? An exploratory study of relationship power and sexual decision-making with African Americans at risk for HIV/STDs. Women \& Health, 39(3), 1-18. doi: 10.1300/J013v39n03_01

Harvey, S. M., Bird, S. T., Galavotti, C., Duncan, E. A., \& Greenberg, D. (2002). Relationship power, sexual decision making and condom use among women at risk for HIV/STDS. Women \& Health, 36(4), 69-84. doi: 10.1300/J013v36n04_06

Hensel, D, \& Fortenberry, J. (2013). A multidimensional model of sexual health and sexual and prevention behavior among adolescent women. Journal of Adolescent Health, 52, 219-227.

Holden, A. E., Shain, R. N., Miller, W. B., Piper, J. M., Perdue, S. T., Thurman, A. R., \& Korte, J. E. (2008). The influence of depression on sexual risk reduction and STD infection in a controlled, randomized intervention trial. Sexually Transmitted Diseases, 35(10), 898-904. doi: 10.1097/OLQ.0b013e31817d7a33

Hulley, SB, Newman, TB, Grady, D, Garber, AM, Baron, RB, \& Browner, WS. (2007). Designing Clinical Research (3rd ed.). Philadelphia: Lippincott, Williams, \& Wilkins.

Hunter, T. S., \& Tilley, D. S. (2015). A grounded theory study of the process used to negotiate condom use among African-American women: Review of literature. Journal of Cultural Diversity, 22(1), 23-29.

Ivankovich, M. B, Leichliter, J.S, \& Douglas Jr., J.M (2013). Measurement of sexual health in the US: an inventory of nationally representative surveys and surveillance systems. Public Health Reports, 128(Supp 1), 62-72.

Jacobs, Robin J, \& Kane, Michael N. (2011). Psychosocial predictors of self-esteem in a multiethnic sample of women over 50 at risk for HIV. Journal of Women \& Aging, 23(1), 23-39.

Jemmott, L. S., Jemmott, J. B., \& O'Leary, A. (2007). Effects on sexual risk behavior and STD rate of brief HIV/STD prevention interventions for African American women in primary care settings. American Journal of Public Health, 97(6), 10341040. doi: 10.2105/ajph.2003.020271 
Johnstone, B, McCormack, G, Yoon, D, \& Smith, M. (2012). Convergent/divergent validity of the Brief Multidimensional Measure of Religiousness/Spirituality: Empirical support for emotional connectedness as a "spiritual" construct. Journal of Religion \& Health, 51, 529-541.

Johnstone, B, Yoon, D. P., Franklin, K. L., Schopp, L., \& Hinkebein, J. (2009). Reconceptualizing the factor structure of the brief multidimensional measure of religiousness/spirituality. Journal of Religion and Health, 48(2), 146-163. doi: 10.1007/s10943-008-9179-9

Lennon, CA, Huedo-Medina, TB, Gerwien, TB, \& Johnson, BT. (2012). A role for depression in sexual risk reduction for women? A meta-analysis of HIV prevention trials with depression outcomes. Social Science \& Medicine, 75(4), 688-698. doi: 10.1016/j.socscimed.2012.01.016

Liu, G., Hariri, S., Bradley, H., Gottlieb, S. L., Leichliter, J. S., \& Markowitz, L. E. (2015). Trends and patterns of sexual behaviors among adolescents and adults aged 14 to 59 years, United States. Sexually Transmitted Diseases, 42(1), 20-26. doi: $10.1097 /$ olq. 0000000000000231

Lorvick, Jennifer, Bourgois, Philippe, Wenger, Lynn D, Arreola, Sonya G, Lutnick, Alexandra, Wechsberg, Wendee M, \& Kral, Alex H. (2012). Sexual pleasure and sexual risk among women who use methamphetamine: A mixed methods study. International Journal of Drug Policy, 23(5), 385-392.

Lutfi, K, Trepka, M , Fennie, K , Ibanez, G, \& Gladwin, H. (2015). Racial residential segregation and risky sexual behavior among non-Hispanic blacks, 2006-2010. Social Science \& Medicine, 140, 95-103. doi: 10.1016/j.socscimed.2015.07.004

Maasoumi, R., Lamyian, M., Montazeri, A., Azin, S. A., Aguilar-Vafaie, M. E., \& Hajizadeh, E. (2013). The sexual quality of life-female (SQOL-F) questionnaire: translation and psychometric properties of the Iranian version. Reproductive Health, 10(25), 25. doi: 10.1186/1742-4755-10-25

Manfrin-Ledet, L., Porche, D. J., \& Westbrook, S. (2015). Relationship of Intimate Partner Violence, HIV Risk Behaviors, and Powerlessness in African-American Women of Childbearing Age. Journal of National Black Nurses' Association, 26(1), 40-49.

Martin, JA , Hamilton, BE, Ventura, SJ , Osterman, MJ , \& Mathews, TJ. (2013). Births: final data for 2011. National Vital Statistics Reports, 62(1), 1-69.

Matsuda, Y, McGrath, J.M, \& Jallo, N. (2012). Use of the Sexual Relationship Power Scale in research: An integrative review. Hispanic Health Care International, 10(4), 175-189. 
McCree, D. H., Wingood, G. M., DiClemente, R., Davies, S., \& Harrington, K. F. (2003). Religiosity and risky sexual behavior in African-American adolescent females. Journal of Adolescent Health, 33(1), 2-8.

McLellan-Lemal, E., O'Daniels, C. M., Marks, G., Villar-Loubet, O., Doherty, I. A., Simpson, C., . . . Borkowf, C. B. (2012). Sexual risk behaviors among AfricanAmerican and Hispanic women in five counties in the Southeastern United States: 2008-2009. Womens Health Issues, 22(1), e9-18. doi: 10.1016/j.whi.2011.06.002

McLellan-Lemal, E., Toledo, L., O'Daniels, C., Villar-Loubet, O., Simpson, C., Adimora, A. A., \& Marks, G. (2013). "A man's gonna do what a man wants to do": African American and Hispanic women's perceptions about heterosexual relationships: a qualitative study. BMC Womens Health, 13, 27. doi: 10.1186/1472-6874-13-27

McMahon, J.M, Volpe, E.M, Klostermann, K, Trabold, N, \& Xue, Y. (2014). A Systematic Review of the Psychometric Properties of the Sexual Relationship Power Scale in HIV/AIDS Research. Archives of Sexual Behavior. doi: 10.1007/s10508-014-0355-6

Melchior, Lisa A., Huba, G. J., Brown, Vivian B., \& Reback, Cathy J. (1993). A Short Depression Index for Women. Educational and Psychological Measurement, 53(4), 1117-1125. doi: 10.1177/0013164493053004024

Merton, R, \& Rossi, A. (1950). Continuities in Social Research. New York: Free Press.

Mincey, K, \& Norris, C. (2014). An Explorative Study of Black Women Sexual Health Throughout Womanhood. Journal of Black Sexuality and Relationships, 1(1), 2743. doi: $10.135 / \mathrm{bsr} .2014 .0008$

Montgomery, BE , Stewart, KE, Yeary, KH , Cornell, CE , Pulley, L , Corwyn, R , \& Ounpraseuth, ST. (2014). Religiosity and sexual risk behaviors among African American cocaine users in the rural South. Journal of Rural Health : Offical Journal of the American Rural Health Association, 30(3), 284-291.

Morse, Janice M, \& Niehaus, Linda. (2009). Mixed method design: Principles and procedures (Vol. 4): Left Coast Pr.

Mosher, W. D., Jones, J., \& Abma, J. C. (2012). Intended and unintended births in the United States: 1982-2010. National Health Statistics Reports, 55(55), 1-28.

Murray, C. C., Hatfield-Timajchy, K., Kraft, J. M., Bergdall, A. R., Habel, M. A., Kottke, M., \& Diclemente, R. J. (2013). In their own words: romantic relationships and the sexual health of young African American women. Public Health Rep, 128 Suppl 1, 33-42.

National Alliance on Mental Illness. (2015). Depression, 2015, from https://www.nami.org/Learn-More/Mental-Health-Conditions/Depression 
National Campaign to Prevent Teen and Unplanned Pregnancy. (2016). Teen pregnancy, 2016, from http://thenationalcampaign.org/why-it-matters/teen-pregnancy

National Institute of Mental Health. (2016). Depression in women Retrieved 2016, from http://www.nimh.nih.gov/health/publications/depression-in-women/index.shtml

National Native American AIDS Prevention Center. (2014). In Community Spirit, from National Native American AIDS Prevention Center

Neblett, RC, Davey-Rothwell, M, Chander, G, \& Latkin, CA. (2011). Social network characteristics and HIV sexual risk behavior among urban African American women. Journal of Urban Health, 88(1), 54-65. doi: 10.1007/s11524-010-9513-x

Nehl, E. J., Elifson, K., DePadilla, L., \& Sterk, C. (2015). Sex Partner Type, Drug Use and Condom Use Self-Efficacy Among African Americans from Disadvantaged Neighborhoods: Are Associations with Consistent Condom Use Moderated by Gender? Journal of Sex Research, 1-11. doi: 10.1080/00224499.2015.1092018

Noar, S. M., Webb, E., Van Stee, S., Feist-Price, S., Crosby, R., Willoughby, J. F., \& Troutman, A. (2012). Sexual partnerships, risk behaviors, and condom use among low-income heterosexual African Americans: a qualitative study. Archives of Sexual Behavior, 41(4), 959-970. doi: 10.1007/s10508-011-9890-6

Noll, J. (2003). Manual for the administration and coding of the Sexual Activities \& Attitudes Questionnaire (SAAQ).

Noll, J, \& Shenk, C. (2013). Teen birth rates in sexually abused and neglected females. Pediatrics, 131(4), e1181-1187. doi: 10.1542/peds.2012-3072

Noll, J, Trickett, P.K, \& Putnam, F.W. (2003). A prospective investigation of the impact of childhood sexual abuse on the develoipment of sexuality. Journal of Consulting and Clinical Psychology, 71(3), 575 - 586.

Nunn, A., Dickman, S., Cornwall, A., Kwakwa, H., Mayer, K. H., Rana, A., \& Rosengard, C. (2012). Concurrent sexual partnerships among African American women in Philadelphia: results from a qualitative study. Sexual Health, 9(3), 288296. doi: 10.1071/sh11099

Office on Women's Health, \& US Department of Health and Human Services. (2016). Screening tests and vaccines: Screening tests for women from http://www.womenshealth.gov/screening-tests-and-vaccines/screening-tests-forwomen/

Painter, T. M., Herbst, J. H., Diallo, D. D., \& White, L. D. (2014). Community-based program to prevent HIV/STD infection among heterosexual black women. Morbidity and Mortality Weekly Reprt Supplements, 63(1), 15-20. 
Pakpour, A., Zeidi, I., Saffari, M., \& Burri, A. (2013). Psychometric properties of the Iranian version of the Sexual Quality of Life Scale among women. Journal of Sexual Medicine, 10, 981-989.

Paxton, KC, Williams, JK, Bolden, S, Guzman, Y, \& Harawa, NT. (2013). HIV Risk Behaviors among African American Women with at-Risk Male Partners. Journal of AIDS and Clinical Research, 4(7), 221-.

Perkins, E. L., Stennis, K. B., Taylor Spriggs, V., Kwegyir-Afful, E. A., \& Prather, A. (2014). Is Knowledge Enough? Considering HIV/AIDS Risk Behaviors and HIV/AIDS Knowledge with African American Women. International Journal of High Risk Behaviors \& Addiction, 3(3), e15038. doi: 10.5812/ijhrba.15038

Pew Research Center. (2015). US demographic groups: Religious affliations, from http://www.pewforum.org/2015/05/12/chapter-4-the-shifting-religious-identityof-demographic-groups/

Pfileger, J , Cook, E, Niccolai, L, \& Connell, C. (2013). Racial/ethnic differences in patterns of sexual risk behavior and rates of sexually transmitted infections among female young adults. American Journal of Public Health, 103(5), 903-909. doi: 10.2105/AJPH.2012.301005

Pfizer Incorporation. (2014). Pfizer patient-reported outcomes Retrieved November 22, 2014, from http://www.pfizerpatientreportedoutcomes.com/therapeuticareas/sexual-health

Piedmont, R, Mapa, A, \& Williams, J. (2007). A factor analyss of the Fetzer/NIA brief multidimensional measure of religiousness/spirituality. Research in the Social Sienctific Study of Religion, 17, 177-196.

Polit, DF, \& Beck, CT. (2012). Nrusing Research: Generating and Assessing Evidence for Nursing Practice (9th ed.). Saratoga Springs: Wolters Kluwer Health | Lippincott Williams \& Wilkins.

Pulerwitz, J, Gortmaker, S.L, \& DeJong, W. (2000). Measuring sexual relationship power in HIV/STD research. Sex Roles, 42(7/8), 637-660.

Quirk, F., Heiman, J., Rosen, R., Laan, E., Smith, M., \& Boolell, M. (2002). Development of a sexual function questionnaire for clinical trials of female sexual dysfunction. Journal of Womens Health Gender Based Medicine, 11(3), 277-289.

Radloff, L.S. (1977). The CES-D Scale: A self-report depression scale for research in the general population. Applied Psychological Measurement, 1(3), 385-401.

Raiford, J. L., Seth, P., \& DiClemente, R. J. (2013). What girls won't do for love: human immunodeficiency virus/sexually transmitted infections risk among young African-American women driven by a relationship imperative. Journal of Adolescent Health, 52(5), 566-571. doi: 10.1016/j.jadohealth.2012.09.006 
Ree, M, French, D , MacLeod, C , \& Locke, V. (2008). Distinguishing Cognitive and Somatic Dimensions of State and Trait Anxiety: Development and Validation of the State-Trait Inventory for Cognitive and Somatic Anxiety (STICSA). Behavioural and Cognitive Psychotherapy, 36(3), 313-332. doi: http://dx.doi.org/10.1017/S1352465808004232

Reid, A. E, Dovidio, J. F, Ballester, E, \& Johnson, B. T. (2014). HIV prevention interventions to reduce sexual risk for African Americans: The influence of community-level stigma and psychological processes. Social Science \& Medicine, 103, 118-125. doi: 10.1016/j.socscimed.2013.06.028

Reiss, I. (1991). Sexual pluralism: Ending America's sexual crisis. SIECUS Report, 19, 59.

Robinson, B. B., Bockting, W. O., Rosser, B. R., Miner, M., \& Coleman, E. (2002). The Sexual Health Model: application of a sexological approach to HIV prevention. Health Education Research, 17(1), 43-57.

Rosner, B. (2011). Fundamentals of Biostatistics (7th ed.). Boston, MA: Brooks/Cole Cengage Learning.

Sales, J. M., DiClemente, R. J., Davis, T. P., \& Sullivan, S. (2012). Exploring why young African American women do not change condom-use behavior following participation in an STI/HIV prevention intervention. Health Education Research, 27(6), 1091-1101. doi: 10.1093/her/cys059

Satcher, D. (2013). Addressing sexual health: Looking back, looking forward. Public Health Reports, 128(Supp 1), 11-114.

Seth, P, Patel, SN, Sales, JM, DiClemente, RJ, Wingood, GM, \& Rose, ES. (2011). The impact of depressive symptomatology on risky sexual behavior and sexual communication among African American female adolescents. Psychology, Health, \& Medicine, 16(3), 346-356. doi: 10.1080/13548506.2011.554562

Sharpe, T, Voute, C, Rose, M, Cleveland, J, Dean, H, \& Fenton, K. (2012). Social determinants of HIV/AIDS and sexually tansmitted diseases among Black women: Implications for health equity. Journal of Women's Health, 21(3), 249254. doi: $10.1089 /$ jwh.2011.3350

Shim, R.S, Baltrus, P, Bradford, L.D, Holden, K.B, Fresh, E, \& Fuller, L.E. (2013). Characterizing depression and comorbid medical conditions in African American Women in a primary care setting. Journal of the National Medical Association, 105(2), 183-191.

Silverman, David. (2011). Interpreting qualitative data: A guide to the principles of qualitative research. 
Smylie, L., Clarke, B., Doherty, M., Gahagan, J., Numer, M., Otis, J., . . Soon, C. (2013). The development and validation of sexual health indicators of Canadians aged 16-24 years. Public Health Reports, 128 Suppl 1, 53-61.

Spitzer, W.O. (1987). State of science 1986: Quality of life and functional status as target variable for research. Journal of Chronic Diseases, 40, 465-471.

Stewart, J. M. (2014). Implementation of evidence-based HIV interventions for young adult African American women in church settings. Journal of Obstetric, Gynecologic, and Neonatal Nursing, 43(5), 655-663. doi: 10.1111/15526909.12494

Stewart, J. M., Sommers, M. S., \& Brawner, B. M. (2013). The Black church, sexual health, and sexuality: a conceptual framework to promote health through faithbased organizations. Family \& Community Health, 36(3), 269-279. doi: 10.1097/FCH.0b013e318292eb2d

Symonds, T., Boolell, M., \& Quirk, F. (2005). Development of a questionnaire on sexual quality of life in women. Journal of Sex \& Marital Therapy, 31(5), 385-397. doi: $10.1080 / 00926230591006502$

Thomas, T. L. , \& Freeman, A. (2011). Project genesis: Self-reported religiosity and spirituality and sexual risk-taking in young African-American women attending a historically African-American college. Journal of National Black Nurses Association, 22(1), 27-35.

U.S. Department of Health and Human Services Health Resources and Services Administration. (2016). Women's preventive services guidelines, 2016, from http://www.hrsa.gov/womensguidelines/

Udell, W., Donenberg, G., \& Emerson, E. (2011). The impact of mental health problems and religiosity on African-American girls' HIV-risk. Cultural Diversity \& Ethnic Minority Psychology, 17(2), 217-224. doi: 10.1037/a0023243

Van Dam, N. T, \& Earleywine, M. (2011). Validation of the Center for Epidemiologic Studies Depression Scale--Revised (CESD-R): pragmatic depression assessment in the general population. Psychiatry Research, 186(1), 128-132. doi:

10.1016/j.psychres.2010.08.018

Ventura, S, Hamilton, B, \& Matthews, T. (2014). National and state patterns of teen births in the United States, 1940-2013. National Vital Statistics Reports, 63(4), 134.

Ventura, S, Mathews, T, Hamilton, B , Sutton, P , Abma, J , \& Centers for Disease Control and Prevention. (2011). Adolescent pregnancy and childbirth - United States, 1991-2008. Morbidity and Mortalitiy Weekly Report, 14(60 Supp), 105108. 
Whyte, J. (2005). The measurement of HIV risk level in African American women who dwell in the Southeastern United States. The Journal of the Association of Nurses in AIDS Care, 16(6), 48-55.

Williams, J, Hirsch, E.S, Anderson, K, Bush, A.L, Goldstein, S.R, Grill, S, . . Marsh, L. (2012). A comparison of nine scales to detect depression in Parkinson disease: which scale to use? Neurology, 78(13), 998-1006. doi:

10.1212/WNL.0b013e31824d587f

Williams, J, Wyatt, GE, \& Wingood, G. (2010). The four Cs of HIV prevention with African Americans: crisis, codoms, culture, and community. Current HIV/AIDS Reports, 7(4), 185-193 doi: 10.1007/s11904-010-0058-0

Williams, T. , Pichon, L. C., Davey-Rothwell, M., \& Latkin, C. A. (2016). Church Attendance as a Predictor of Number of Sexual Health Topics Discussed Among High-Risk HIV-Negative Black Women. Archives of Sexual Behavior, 45(2), 451458. doi: 10.1007/s10508-015-0506-4

Williams, T., Pichon, L. C., Latkin, C. A., \& Davey-Rothwell, M. (2014). Practicing What is Preached: The Relationship between Congregational Support and HIV Prevention Behaviors among Black Women. Journal of Community Psychology, 42(3), 365-378. doi: 10.1002/jcop.21615

Wingood, G. M., \& DiClemente, R. J. (2008). The ADAPT-ITT model: a novel method of adapting evidence-based HIV Interventions. Journal of Acquired Immune Deficiency Syndromes, 47 Suppl 1, S40-46. doi: 10.1097/QAI.0b013e3181605df1

Wingood, G. M., DiClemente, R. J., Mikhail, I., Lang, D. L., McCree, D. H., Davies, S. L., . . . Saag, M. (2004). A randomized controlled trial to reduce HIV transmission risk behaviors and sexually transmitted diseases among women living with HIV: The WiLLOW Program. Journal of Acquired Immune Deficiency Syndromes, 37 Suppl 2, S58-67.

Wingood, G. M., Robinson, L. R., Braxton, N. D., Er, D. L., Conner, A. C., Renfro, T. L., ... Diclemente, R. J. (2013). Comparative effectiveness of a faith-based HIV intervention for African American women: importance of enhancing religious social capital. American Journal of Public Health, 103(12), 2226-2233. doi: 10.2105/ajph.2013.301386

Wingood, G. M., Scd, \& DiClemente, R. J. (2000). Application of the theory of gender and power to examine HIV-related exposures, risk factors, and effective interventions for women. Health Education \& Behavior, 27(5), 539-565.

World Health Organization. (1975). Education and treatment in human sexuality: The training of health professionals. WHO Technical Report Series No. 572. Geneva: World Health Organization. 
World Health Organization. (2002). Defining sexual health: Report of a technical consultation on sexual health 28-31 January 2002, Geneva.

World Health Organization. (2010). Measuring sexual health: Conceptual and practical considerations and related indicators. Geneva, Switzerland.

World Health Organization. (2015). Defining sexual health, from http://www.who.int/reproductivehealth/topics/sexual health/sh_definitions/en/

Wyatt, GE, Williams, JK, \& Malebranche, D. (2012). Are cultural values and beliefs included in U.S. based HIV interventions? Preventive Medicine, 55(5), 362-370. doi: 10.1016/j.ypmed.2011.08.021

Zembe, Y. Z., Townsend, L., Thorson, A., Silberschmidt, M., \& Ekstrom, A. M. (2015). Intimate Partner Violence, Relationship Power Inequity and the Role of Sexual and Social Risk Factors in the Production of Violence among Young Women Who Have Multiple Sexual Partners in a Peri-Urban Setting in South Africa. PLoS One, 10(11), e0139430. doi: 10.1371/journal.pone.0139430 


\section{APPENDIX A. INSTITUTIONAL REVIEW BOARD LETTER OF APPROVAL}

\begin{tabular}{lr}
\hline THE UNIVERSITY OF TENNESSEE \\
Health Science Center
\end{tabular}

May 01, 2015

Mona N Wicks, Ph.D.

UTHSC - CON - Research Programs

645 Alexander Building

877 Madison Avenue

Memphis, TN 38163--0000

Melody N Waller, PhDc, MSN, RN

UTHSC - CON - Research Programs

1013920 Madison Building

920 Madison Avenue

Memphis, TN 38163

Re: 15-03752-XP

Study Title: Factors Associated with Sexual Health and Risk Behavior in African American Women: A Mixed Methods Study

Dear Ms. Waller:

The Administrative Section of the UTHSC Institutional Review Board (IRB) has received your written acceptance of and/or response dated 05/01/2015 12:45:23 PM CDT to the provisos outlined in our correspondence of 03/23/2015 and 04/28/2015 concerning the above referenced project. The IRB determined that your application is eligible for expedited review under $45 \mathrm{CFR}$ 46.110(b)(1) categories (6) and (7). The IRB has reviewed these materials and determined that they do comply with proper consideration for the rights and welfare of human subjects and the regulatory requirements for the protection of human subjects. Therefore, this letter constitutes full approval by the IRB of your application (version 1.4) as submitted including:

- Sexual Health Study Flyer dated 05/01/2015 (stamped IRB approved May 1, 2015)

- Facebook Flyer(3)_dated 05/01/2015 (stamped IRB approved May 1, 2015)

- Telephone Screening Script dated 04/05/2015 (stamped IRB approved May 1, 2015)

- Recruitment Email dated 04/05/2015 (stamped IRB approved May 1, 2015)

- Participant Demographic Form dated 04/05/2015 (stamped IRB approved May 1, 2015)

- Focus Group Interview Guide dated 04/08/2015 (stamped IRB approved May 1, 2015)

- Sexual Relationship Power Scale dated 04/03/2015 (stamped IRB approved May 1, 2015) 
Page $\mathbf{2}$ of 2

- The Center for Epidemiologic Studies Depression Scale Revised (CESD-R) dated

○ 04/03/2015 (stamped IRB approved May 1, 2015)

- The Brief Multidimensional Measure of Religiousness/Spirituality (BMMRS) dated

○ 04/03/2015 (stamped IRB approved May 1, 2015)

- The Sexual Activities and Attitudes Questionnaire (SAAQ) dated 04/03/2015 (stamped IRB approved May 1, 2015)

- The Sexual Quality of Life Female (SQOL-F) Questionnaire dated 04/03/2015 (stamped IRB approved May 1, 2015); and

- Consent form dated April 5, 2015 (stamped IRB approved May 1, 2015). The UTHSC IRB stamped-approved consent form must be used to enroll prospective subjects in the study.

Approval of this study will be valid from May 01, 2015 to 03/10/2016.

The IRB has determined that the informed consent form, incorporating the authorization of subjects to use their protected health information in research, complies with the federal privacy regulations as specified in 45 CFR 160 and 45 CFR 164.

\section{You must submit a copy of the Certificate of Confidentiality (COC) to the IRB when it is obtained. In addition, subjects could not be recruited until the IRB acknowledged the $\mathrm{CoC}$ in the file.}

In the event that subjects are to be recruited using solicitation materials, such as brochures, posters, web-based advertisements, etc., these materials must receive prior approval of the IRB. Any revisions in the approved application must also be submitted to and approved by the IRB prior to implementation. In addition, you are responsible for reporting any unanticipated serious adverse events or other problems involving risks to subjects or others in the manner required by the local IRB policy.

Finally, re-approval of your project is required by the IRB in accord with the conditions specified above. You may not continue the research study beyond the time or other limits specified unless you obtain prior written approval of the IRB.

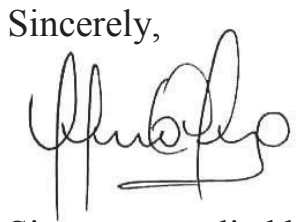

Signature applied by Terrence F Ackerman on 05/01/2015 03:23:53 PM CDT 


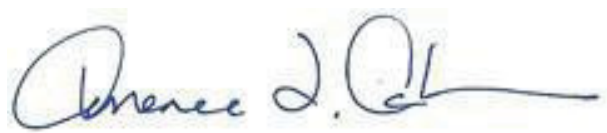

Signature applied by Virginia D Calvo Torres on 05/01/2015 03:23:05 PM CDT

Virginia Calvo-Torres, BS, CCRP, CIM

Ph.D. IRB Compliance Advisor

UTHSC IRB
Terrence F. Ackerman,

Chairman

UTHSC IRB 


\section{APPENDIX B. INSTITUTIONAL REVIEW BOARD APPROVED CONSENT FORM}

Main Consent Form

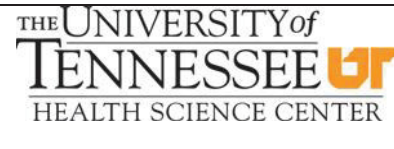

\section{TITLE: Factors Associated with Sexual Health and Risk Behavior in African American Women: A Mixed Methods Study}

PRINCIPAL INVESTIGATOR:

CO-INVESTIGATOR(S):
Melody N. Waller PhD candidate, MSN, RN 920 Madison Ave. Suite 1013

Memphis, TN 38163

Mona Wicks, $\mathrm{PhD}, \mathrm{RN}$

J. Carolyn Graff $\mathrm{PhD}, \mathrm{RN}$

Betsy Tolley, PhD, MS, BS

\section{INTRODUCTION:}

You are being given the opportunity to participate in this research study. The purpose of this consent form is to help you decide if you want to be in the research study. This consent form may contain words that you do not understand. Please ask the investigator to explain any words or information that you do not clearly understand. We encourage you to talk with your family and friends before you decide to take part in this research study. Please tell the investigator if you are taking part in another research study.

The purpose of this study is to better understand sexual health and behaviors of African American women. This study will also seek to explore African American women's perception of sexual health and sexual risk behavior. Sexual risk behaviors or behaviors thought to increase risk of acquiring sexually transmitted infections, Human Immunodeficiency Virus (HIV), or unintended pregnancy will also be investigated as part of this study's purpose. You will be asked to complete questionnaires about sexual wellbeing, sexual attitudes and activities, religiosity and spirituality, depressive symptomology, and sexual relationship power. Your name or other personally identifying information will not be associated with your responses. Any data reported from participant response will be reported as a whole and will not identify you.

A total of 50 subjects are being sought for participation in this study.

The study will take place at the University of Tennessee Health Science Center at 920 Madison Avenue in either Suite \# 923, 953, or 1013 in Memphis, TN 38163. 
Your participation in this study will be expected to last for 2 hours. It will take about 1 to $1 \frac{1}{2}$ hours to complete the study questionnaires.

You may also be asked to participate in focus group interviews to share your thoughts and views on sexual health. A focus group is a group interview led by two facilitators. One facilitator asks questions while the other facilitator takes notes. Focus group interviews will be scheduled within six weeks of initial study participation. Your participation in focus group interviews will be expected to last for 2 hours. You may refuse to participate in any aspect of this research study. This study will be conducted over a duration of 6 months to one year.

\section{PROCEDURES TO BE FOLLOWED:}

Initial Study Visit (this will take 1 to $1 \frac{1}{2}$ hours):

- $\quad$ The primary researcher will speak with you to confirm study eligibility and willingness to participate.

- The primary researcher will review the informed consent form.

- $\quad$ You will be asked to complete a questionnaire regarding your background, social, and medical history.

- You will then be asked to complete five questionnaires about sexual well-being, sexual attitudes and activities, religiosity and spirituality, depressive symptomology, and sexual relationship power. Questionnaires to be completed have been described below:

\begin{tabular}{|l|c|l|}
\hline \multicolumn{1}{|c|}{ Questionnaire } & Number of Questions & \multicolumn{1}{c|}{ Description } \\
\hline $\begin{array}{l}\text { Sexual Quality of Life } \\
\text { Questionnaire - Female }\end{array}$ & 18 & $\begin{array}{l}\text { To measure sexual well- } \\
\text { being through personal } \\
\text { and relationship issues. }\end{array}$ \\
\hline $\begin{array}{l}\text { Sexual Activities and Attitudes } \\
\text { Questionnaire }\end{array}$ & 44 & $\begin{array}{l}\text { To evaluate sexual activity } \\
\text { to include: birth control } \\
\text { use, sexual behaviors, } \\
\text { sexual partners; and sexual } \\
\text { attitudes. }\end{array}$ \\
\hline $\begin{array}{l}\text { Brief Multidimensional } \\
\text { measure of } \\
\text { Religiousness/Spirituality }\end{array}$ & 38 & $\begin{array}{l}\text { To compare features of } \\
\text { spirituality and religion, } \\
\text { while identifying their } \\
\text { connection to health. }\end{array}$ \\
\hline $\begin{array}{l}\text { Center for Epidemiologic } \\
\text { Studies Depression Scale } \\
\text { Revised }\end{array}$ & 20 & $\begin{array}{l}\text { To identify symptoms of } \\
\text { depression and depressive } \\
\text { disorder. }\end{array}$ \\
\hline $\begin{array}{l}\text { Sexual Relationship Power } \\
\text { Scale }\end{array}$ & 23 & $\begin{array}{l}\text { To measure issues that } \\
\text { occur within relationships } \\
\text { and/or relationship habits. }\end{array}$ \\
\hline
\end{tabular}


6. In the case that the questionnaire used to identify depression or depressive symptoms suggest that you may hurt yourself, a referral will be made to your medical care provider. If you do not have a medical care provider, a referral will be made to the Comprehensive Counseling Network, a local community agency that offers mental health services in Shelby county.

- $\quad$ You may also be asked to participate in a 2-hour focus group interview that will be scheduled within six weeks of your initial study visit.

Focus Group Visit (this will take up to 2 hours):

- $\quad$ Focus group interviews will take place within six weeks of your initial study visit.

- The primary researcher will speak with you to re-confirm study eligibility and willingness to participate.

- $\quad$ You will be asked questions and allowed to respond freely within a group of 8 10 study participants.

- $\quad$ Questions asked during focus group interviews will look at your views or thoughts about sexual health; emotional and relationship issues that influence your sexual health; and behaviors that contribute to your sexual health.

- $\quad$ You will be asked to respect the confidentiality of other focus group participants by treating information revealed during focus group sessions as private.

- $\quad$ Focus group interviews will be recorded using both an iPad and digital voice recorder. Audio and digital recordings from focus groups will be used to examine interview responses.

Focus Group Participation:

Please mark " $X$ " in the box below to indicate whether you are interested or not interested in participating in a focus group interview.

I am interested in participating in a focus group interview as part of this research study.

\section{Research Subject Initials}

I am NOT interested in participating in a focus group interview as part of this research study.

$\overline{\text { Research Subject Initials }}$

All the procedures described in this consent form are for research purposes only.

\section{RISKS ASSOCIATED WITH PARTICIPATION:}

Both psychological and legal risks may occur from participation in this study. Potential risks of study procedures may include: 
- $\quad$ Psychological risk may be posed due to potential emotional discomfort associated with the study's subject matter. You will be asked questions of a highly personal nature regarding fairly sensitive topics (sexual behavior, sexually transmitted infections (STIs), intimate partner violence) either through completion of questionnaires or focus group interviews.

- Legal risks may be encountered from the completion of questionnaires that may involve disclosure of illegal activity. The admission of such information could pose legal risk if investigators are required by law (search warrant or subpoena) to disclose information.

Focus Group Participation:

- Psychological risks may also be posed during focus group interviews due to emotional discomfort associated with revealing personal information on sensitive topics with others.

- Informational risks and potential breaches of confidentiality may be posed due to the sharing of information during focus group interviews; the study investigator cannot guarantee the confidentiality of data being discussed.

- Social risks may be posed if other focus group members disagree with information disclosed during interviews.

- Potential breaches of confidentiality and legal risks may be posed from the disclosure of sensitive information or illegal activity on voice and digital recordings.

There is the potential risk of loss of confidentiality. Every effort will be made to keep your information confidential; however, this cannot be guaranteed.

The research may involve risks to you, which are currently unforeseeable. You will be told about any new information that might change your decision to be in this study. You may be asked to sign a new consent form if this occurs.

\section{Questionnaires/Surveys:}

Completion of the Sexual Quality of Life Questionnaire, Sexual Activities and Attitudes Questionnaire, Brief Multidimensional Measure of Religiousness/Spirituality, Center for Epidemiologic Studies Depression Scale Revised, and the Sexual Relationship Power Scale may make you feel uncomfortable or cause troublesome feelings or emotions. You may refuse to answer any of the questions and you may take a break at any time during the study.

\section{BENEFITS ASSOCIATED WITH PARTICIPATION:}

You will not receive any benefits from being in this study. The results of this study may be used to help women better understand sexual health and health behaviors in the future by sharing your experiences. Your participation in this study may help to identify issues that can contribute to women achieving a state of sexual well-being and participating in healthy sexual behaviors. 


\section{ALTERNATIVES TO PARTICIPATION:}

You do not have to participate in this study.

You will not have to undergo the following procedures if you do not take part in this study:

- Complete 1 questionnaire regarding your background, social, and medical history.

- Complete additional questionnaires about sexual well-being, sexual attitudes and activities, religiosity and spirituality, depressive symptomology, and sexual relationship power.

- $\quad$ Asked to participate in a focus group interview that will be scheduled within six weeks of your initial study visit.

- $\quad$ Asked questions and allowed to respond freely within a focus group of $8-10$ study participants.

\section{CONFIDENTIALITY:}

\section{Research records/specimens}

All of your paper research records will be stored in locked file cabinets and will be accessible only to research personnel.

All your electronic research records will be computer password protected and accessible only to research personnel.

\section{Presentations/Publications}

You will not be identified in any presentations or publications based on the results of this research study.

\section{Authorization to Use and Disclose Information for Research Purposes}

Under federal privacy regulations, you have the right to decide who can review and copy your personal health information (called "protected health information" or PHI). PHI collected in this study may include information such as:

- $\quad$ Past and present medical records

- $\quad$ Records about your study visits

- $\quad$ Records about phone calls made as part of this research

- $\quad$ Research records

By signing this consent form, you are giving your permission for the study investigator and the study staff at the University of Tennessee to get your PHI from your doctor and/or facilities where you have received health care. They may also share your PHI with:

- The Institutional Review Board (IRB) at the University of Tennessee Health Science Center

Your PHI will be used until the study is completed. 
You may withdraw or take away your permission to use and disclose your PHI at any time. You do this by sending written notice to the study investigator. If you withdraw your permission, you may not be able to stay in the study.

When you withdraw your permission, no new PHI will be gathered after that date. However, information that has already been gathered may still be used and given to others. The federal regulations allow you to review or copy your PHI that is used in this study.

\section{Certificate of Confidentiality}

To help us further protect your privacy, the investigators have obtained a Confidentiality Certificate from the Department of Health and Human Services (DHHS).

With this certificate, the investigators cannot be forced (for example, by court subpoena) to disclose research information that may identify you in any Federal, State, or local civil, criminal, administrative, legislative, or other proceedings. Disclosure will be necessary, however, upon request of DHHS for audit or program evaluation purposes.

The Confidentiality Certificate does not prevent you or a member of your family from voluntarily releasing information about yourself or your involvement in this research. Note, however, that if an insurer or employer learns about your participation and obtains your consent to receive research information, then the investigator may not use the Certificate of Confidentiality to withhold this information. This means that you and your family must also actively protect your own privacy.

Finally, the investigator is not prevented from taking steps, including disclosure of your research information to authorities, in order to prevent serious harm to yourself or others.

\section{COMPENSATION AND TREATMENT FOR INJURY:}

You are not waiving any legal rights or releasing the University of Tennessee or its agents from liability for negligence. In the event of physical injury resulting from research procedures, the University of Tennessee does not have funds budgeted for compensation for medical treatment. Therefore, the University of Tennessee does not provide for treatment or reimbursement for such injuries.

If you are injured or get sick as a result of being in this study, call the study investigator immediately. The study investigator will provide you with a subsequent referral to appropriate health care facilities.

If you are injured or get sick as a result of being in this study, you and/or your insurance will be billed for the costs associated with this medical treatment.

No compensation will be available to you for any extra expenses that you may have as the result of research related physical injuries, such as additional hospital bills, lost wages, travel expenses, etc. 
No compensation will be available to you for any non-physical injuries that you may have as a result of research participation, such as legal problems, problems with your finances or job, or damage to your reputation.

\section{QUESTIONS:}

Contact Melody Waller, PhD candidate, MSN, RN, principal investigator, at (901) 2621430 if you have questions about your participation in this study, or if you have questions, concerns, or complaints about the research.

If you feel you have had a research-related injury, contact Melody Waller, PhD candidate, MSN, RN, principal investigator, at (901) 262-1430 (cell) 24-hours a day/7days a week.

You may contact Terrence F. Ackerman, Ph.D., UTHSC IRB Chairman, at 901-4484824 , or visit the IRB website at http://www.uthsc.edu/research/research_compliance/IRB/participant_complaint.php if you have any questions about your rights as a research subject, or if you have questions, concerns, or complaints about the research.

\section{PAYMENT FOR PARTICIPATION:}

You will receive a \$20 gift card to Target or Wal-Mart at the completion of each study visit. If you complete all the study visits, you will receive a total of Target or Wal-Mart gift cards worth $\$ 40$. If you do not complete both study visits, you will only be paid for the visit you have completed.

\section{COSTS OF PARTICIPATION:}

There are no costs to you for participating in this study.

\section{VOLUNTARY PARTICIPATION AND WITHDRAWAL:}

If you decide to stop being part of the study, you should tell your study investigator, and any information that you have already provided will be kept in a confidential manner.

The study investigator may stop your participation in this research study without your consent for any of the following reasons:

- If you do not show up for visits

- If you refuse to complete any of the questionnaires.

Your participation in this research study is voluntary. You may decide not to participate or you may leave the study at any time. Your decision will not result in any penalty or loss of benefits to which you are entitled. 
If you are a student of the University of Tennessee Health Science Center participating or not participating in this study will in no way influence your grade in any course. If you are an employee of the University of Tennessee Health Science Center participating or not participating in this study will not affect your employment status.

\section{Assent Discussion for Adult Subjects}

\section{CONSENT OF SUBJECT:}

You have read or have had read to you a description of the research study as outlined above. The investigator or his/her representative has explained the study to you and has answered all the questions you have at this time. You knowingly and freely choose to participate in the study. A copy of this consent form will be given to you for your records.

Signature of Research Subject (18 years + )

$\overline{\text { Date }} \overline{\text { Time }}$

\section{Printed Name of Adult Research Subject}

Signature of Person Obtaining Consent

$\overline{\text { Date }} \overline{\text { Time }}$

\section{Printed Name of Person Obtaining Consent}

In my judgment, the subject has voluntarily and knowingly given informed consent and possesses the legal capacity to give informed consent to participate in this research study.

Signature of Investigator

$\overline{\text { Date }} \overline{\text { Time }}$

[Prepared: April 5, 2015]

Subject Initials 
APPENDIX C. PARTICIPANT DEMOGRAPHIC FORM

Factors Associated with Sexual Health and Risk Behavior in African American Women: A Mixed Methods Study

Study ID:

Visit Date:

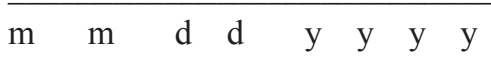

Instructions: Please mark " $X$ " for the answer choice with which you MOST CLOSELY identify with.

1. Age (in years):

$\square 18-25 \quad \square 46-55 \square 26-35 \square \square 6-65$

$\square 36-45 \quad \square 66$ and over

2. Relationship Status:

$\square$ Single $\square$ Divorced $\square$ Living with Partner

$\square$ Married $\square$ Widowed

3. Sexual Orientation/Preference:

$\begin{array}{lll}\square \text { Heterosexual } & \square \text { Gay } & \square \text { Bisexual } \\ \square \text { Lesbian } & \square \text { Transgender } & \square \text { Queer }\end{array}$

4. Education Level (Mark the highest level of education completed):

$\square$ Grade $0-8 \quad \square 2$ or 4 year College Degree

$\square$ Grade 9 - $12 \quad \square$ Post Graduate Degree

$\square$ High School Graduate/GED

Waller Dissertation-Study Enrollment/Init

NUMBER: 15-03752-XP

TuUNIVESITY of

IRB APPROVAL DATE: 05/01/2015 


\section{Employment Status:}

$\square$ Formally Employed $\quad \square$ Unemployed
Self-Employed

\section{Type of Health Insurance Coverage:}

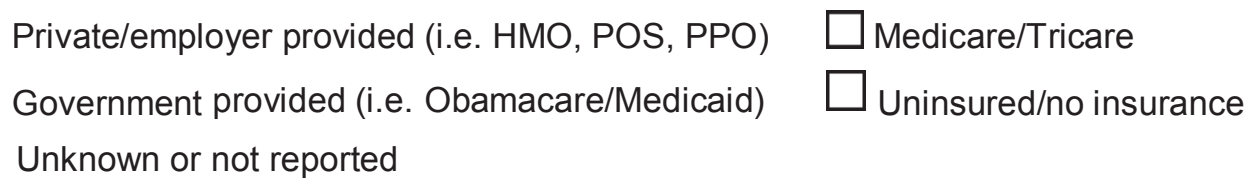

\section{Reproductive History/Information}

7. Last Visit with Reproductive Health Care Provider (i.e. OB/GYN, Women's Health Nurse Practitioner):

$\square$ Less than 1 year ago $\square 4-5$ years ago

$\square 2-3$ years ago $\quad \square$ Over 5 years ago

\section{Reproductive Health Status:}

Pre-menopausal (i.e. regular monthly menstrual periods)

$\square$ Peri-menopausal (i.e. irregular - a change of 7 or more days and/or a space of 60 or more days between menstrual periods)

$\square$ Post-menopausal (i.e. 12 consecutive months without a menstrual period)

$\square$ Unknown or other

9. Contraceptive Use (Pregnancy prevention device/method):
$\square$ Birth Control Pill
$\square$ Vaginal Ring $\square$ Withdrawal
$\square$ Birth Control Patch $\square$ Intrauterine Device (IUD)
$\square$ Birth Control Implant $\square$ Male Condom $\square$ None 


\section{Social History}

10. Substance Use (Mark the answer choice related to your involvement with substances within the past 12 months):

Alcohol - Number of drinks per week:

Tobacco/cigarettes - Number of packs per day: Illicit/illegal drug use:

$\square$ Marijuana

$\square$ Cocaine

$\square$ Prescription medication (not taken as directed by provider)

$\square$ Other - Specify: $\square$ Heroin

$\square$ Methamphetamine

\section{General Health Information}

11. Medical Conditions (Mark an answer if you have ever been diagnosed by a physician/health care provider with any of the following conditions):

$\square$ Heart Disease

High Blood Pressure

$\square$ Diabetes/High Blood Sugar

Vision/Hearing Disorder Disorder

Blood Disorders 
13. Family History of Mental Health Conditions (Mark an answer if anyone in your family has ever been diagnosed by a physician/health care provider with any of the following conditions):

$\square$ Depression

$\square$ Bipolar Disorder

Anxiety

TEUNIVERSITYof

Waller Dissertation-Study Enrollment/Init $\square$ Schizophrenia

$\square$ Eating Disorders

$\square$ Other - Specify:

NUMBER: 15-03752-XP

IRB APPROVAL DATE: 05/01/2015 


\section{APPENDIX D. DEFINITIONS OF QUALITATIVE CODES AND CATEGORIES}

\begin{tabular}{|c|c|}
\hline Qualitative Categories/Codes & Definition \\
\hline Assimilation & $\begin{array}{l}\text { Familiarization or adjustment to sexual culture, } \\
\text { confidence, or well-being }\end{array}$ \\
\hline Generational & $\begin{array}{l}\text { Familiarization or adjustment to sexual culture, } \\
\text { confidence, or well-being characterized by varying } \\
\text { age groups or life stage }\end{array}$ \\
\hline Emotional & $\begin{array}{l}\text { Intuitive factors developed by and/or displayed } \\
\text { through a person's feelings }\end{array}$ \\
\hline $\begin{array}{l}\text { Depression/Depressive } \\
\text { Symptomology }\end{array}$ & $\begin{array}{l}\text { Ongoing feelings of sadness that interrupt typical } \\
\text { daily activities }\end{array}$ \\
\hline Feelings & $\begin{array}{l}\text { One's display of emotion, mental state, or intuitive } \\
\text { impression }\end{array}$ \\
\hline Fears & One's display of concern or apprehension \\
\hline Partner's Feelings & $\begin{array}{l}\text { A sexual or intimate partner's display of emotion, } \\
\text { mental state, or intuitive impression }\end{array}$ \\
\hline Expression of Sexual Health & $\begin{array}{l}\text { Showing through participation in various behaviors } \\
\text { or practices an acknowledgment of sexual health }\end{array}$ \\
\hline Influence & $\begin{array}{l}\text { Determinant factors that may serve to guide or alter } \\
\text { one's sexual decision-making, behavior, or } \\
\text { practices }\end{array}$ \\
\hline Approach & $\begin{array}{l}\text { The perspective taken to guide or alter one's sexual } \\
\text { decision-making, behavior, or practices }\end{array}$ \\
\hline Family-Work Demands & $\begin{array}{l}\text { Family and work responsibilities required to } \\
\text { maintain personal balance that also serve to guide } \\
\text { or alter one's sexual decision-making, behavior, or } \\
\text { practices }\end{array}$ \\
\hline From Others & $\begin{array}{l}\text { The effects from other individuals that may serve to } \\
\text { guide or alter one's sexual decision-making, } \\
\text { behavior, or practices }\end{array}$ \\
\hline Societal & $\begin{array}{l}\text { The effects from society or culture that may serve } \\
\text { to guide or alter one's sexual decision-making, } \\
\text { behavior, or practices }\end{array}$ \\
\hline Multi-dimensional & $\begin{array}{l}\text { A complex concept composed of or influenced by } \\
\text { multiple factors including: physical, emotional, } \\
\text { relational, and/or sexual components }\end{array}$ \\
\hline
\end{tabular}




\begin{tabular}{|c|c|}
\hline Qualitative Categories/Codes & Definition \\
\hline Relational & $\begin{array}{l}\text { Instinctive factors developed by or contributing to } \\
\text { the connection between an individual and another } \\
\text { person }\end{array}$ \\
\hline Relationship Dynamics & $\begin{array}{l}\text { Circumstances that underlie or characterize the } \\
\text { composition and/or connection between an } \\
\text { individual and their sexual or intimate partner }\end{array}$ \\
\hline Religiosity/Spirituality & $\begin{array}{l}\text { Relating to or demonstrating one's belief or } \\
\text { devotion to God, a higher being, or one's spirit or } \\
\text { soul as an entity separate from their physical body }\end{array}$ \\
\hline Self-sufficiency & $\begin{array}{l}\text { Factors contributing to or characterizing one's } \\
\text { independence and reliance on self for personal } \\
\text { sustainability }\end{array}$ \\
\hline Decision-making & $\begin{array}{l}\text { Factors involved in the process of selecting or } \\
\text { choosing between available alternatives that } \\
\text { contribute to one's independence and reliance on } \\
\text { self for personal sustainability }\end{array}$ \\
\hline Personal standards/ideals & $\begin{array}{l}\text { Principles that guide an individual's sexual beliefs, } \\
\text { behavior, and practices by contributing to or } \\
\text { characterizing one's independence and reliance on } \\
\text { self for personal sustainability }\end{array}$ \\
\hline Self-concept & $\begin{array}{l}\text { One's perception of themselves which serves to } \\
\text { contribute to or characterize one's independence } \\
\text { and reliance on self for personal sustainability }\end{array}$ \\
\hline Sexual & $\begin{array}{l}\text { Instinctive factors developed by or contributing to } \\
\text { one's sexuality and/or the physical connection } \\
\text { between an individual and their intimate partner }\end{array}$ \\
\hline Emotional Barriers & $\begin{array}{l}\text { Obstacles generated by one's feelings that may } \\
\text { hinder the physical connection between an } \\
\text { individual and their sexual or intimate partner }\end{array}$ \\
\hline Physical Barriers & $\begin{array}{l}\text { Obstacles generated by a physiological condition or } \\
\text { one's physical state that may hinder the physical } \\
\text { connection between an individual and their sexual } \\
\text { or intimate partner }\end{array}$ \\
\hline
\end{tabular}




\section{VITA}

Melody Norris Waller, born in 1979, received a Bachelor's of Science in Nursing degree from the University of Tennessee at Chattanooga in 2001. After gaining experience as a staff nurse in maternity nursing, she obtained a Master's of Science in Nursing degree focusing on Nursing Education with a concentration in Maternal-Child Health from the University of Memphis in 2009. Her fifteen years of experience within the nursing profession includes work in the areas of obstetrical nursing, maternal-fetal, women's health, and kidney transplant research, and nursing education. As a nurse educator, Melody's teaching responsibilities include providing didactic and clinical instruction in the areas of Maternal-Child Nursing, Medication Safety, and EvidenceBased Practice.

Melody has presented her research at several regional meetings, including Southern Nursing Research Society (SNRS) Annual Conferences and the National Association of Neonatal Nurses Annual Education Conference. Her poster presentation entitled "The shift towards sexual health: A comprehensive approach to address the wellbeing of African-American women placed $2^{\text {nd }}$ for student research posters in the SNRS Minority Health category. Additional honors include receiving the Johnson \& Johnson/American Association of Colleges of Nursing Minority Nurse Faculty Scholarship Award in 2013 and 2014. Melody is also a proud member of the Association of Women's Health, Obstetric, and Neonatal Nurses, Sigma Theta Tau International Nursing Honor Society, and Southern Nursing Research Society.

Melody will receive her Doctor of Philosophy degree in Nursing Science in May of 2016. Her dissertation research focused on sexual health and risk behavior in African American women. She is currently an instructor at the University of Tennessee Health Science Center's College of Nursing in Memphis, TN. 